\title{
EXPERIMENTAL INVESTIGATION OF THERMAL DIFFUSION IN BINARY AND TERNARY HYDROCARBON MIXTURES
}

\author{
by \\ Seyyed Arash Mousavi \\ B. Sc., Mechanical Engineering, Shahid Chamran University of Ahwaz, 2008 \\ M. Sc., Mechanical Engineering, Razi University, 2011
}

\author{
A dissertation \\ presented to Ryerson University \\ in partial fulfillment of the \\ requirements for the degree of \\ Doctor of Philosophy \\ in the Program of \\ Mechanical Engineering
}

Toronto, Ontario, Canada, 2016

CSeyyed Arash Mousavi 2016 


\section{Author's Declaration}

I hereby declare that I am the sole author of this dissertation. This is a true copy of the dissertation, including any required final revisions, as accepted by my examiners.

I authorize Ryerson University to lend this dissertation to other institutions or individuals for the purpose of scholarly research.

I further authorize Ryerson University to reproduce this dissertation by photocopying or by other

means, in total or in part, at the request of other institutions or individuals for the purpose of scholarly research.

I understand that my dissertation may be made electronically available to the public. 


\title{
Abstract \\ EXPERIMENTAL INVESTIGATION OF THERMAL DIFFUSION IN BINARY AND TERNARY HYDROCARBON MIXTURES
}

\author{
Doctor of Philosophy, Mechanical Engineering, Ryerson University, January 2016 \\ Seyyed Arash Mousavi
}

In a multi-component liquid mixture, the process of disassociation of the components induced by thermal gradient is called thermal diffusion or Soret effect. This effect plays a crucial role in separation of the components in hydrocarbon mixtures of oil. Accordingly, the main goal of this study is to experimentally investigate the Soret effect in binary and ternary hydrocarbon mixtures. Optical interferometry technique with Mach-Zehnder scheme was used to conduct the experiments. The interferometry techniques are not intrusive and the separation of the components in the mixture is not affected by the measurement instrument. A Soret cell is defined as a cubic cavity where the sample mixture is placed in it and, the separation of the components takes place in the cell by heating it from the above. Soret cells are used in convectionless experiments and natural convections are undesirable. The Soret cell used in space experiments was re-designed and optimized for ground-based experiments to avoid the natural convections. Computational studies were made on the both cells to obtain the temperature and velocity fields. Then a set of thermal diffusion experiments conducted in order to compare the performance of the cells. The results shows that the induced convective motions in the second cell are significantly weaker than those in the previous cell which is desirable.

In the next step, the effect of the inclination of the cell on the thermal diffusion was studied. First numerical analysis was made to find the velocity and temperature fields in different inclinations and then a set of experiments was performed and the concentration distribution of the components in a binary mixture in different inclinations of the cell was found.

Finally, ground based experiments were performed to study the thermal diffusion in five ternary hydrocarbon mixtures. Optical interferometry with Mach-Zehnder scheme using two laser sources with different wavelengths was used. The Soret information of one of the mixtures is available in the literature and this mixture was studied here to validate the present experimental setup. The temperature and concentration of the mixtures were measured successfully in the Soret cell and a table of the measured Soret coefficient were provided. 


\section{Acknowledgements}

I would like to express my gratitude to my supervisor, Prof Ziad Saghir for his unequivocal support during my $\mathrm{PhD}$ studies. I gratefully acknowledge his patience and helpful advice. $\mathrm{He}$ guided me thoroughly step-by-step during my research and the writing of my thesis.

A very special thanks goes to Dr. Tooraj Yousefi for his nonstop support as I conducted my research. Dr. Yousefi has been advising me since my master studies, and his motivation and encouragement during my MSc and $\mathrm{PhD}$ studies were unbelievably helpful.

I also acknowledge the financial support from the Canadian Space Agency and Natural Sciences \& Engineering Research Council of Canada (NSERC).

Last but not least, I would like to express my gratitude to my family. The most energetic inspiration that kept me moving forward come from my parents and the unconditional love that they have always given. Words cannot express my gratitude towards them. I thank all of my siblings: Babak, Siamak, Hassan, Yalda, and Sheida for their love, patience, and encouragement which have been given from the other side of the world. This dissertation is dedicated to my family. 


\section{Table of Contents}

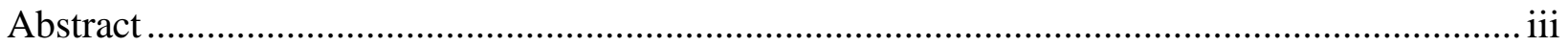

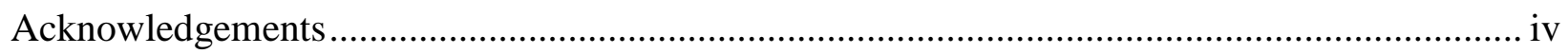

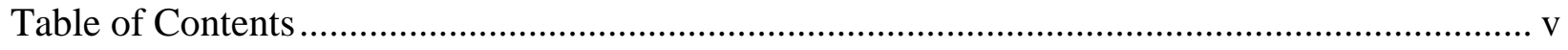

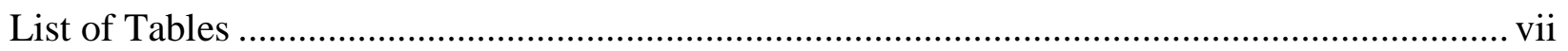

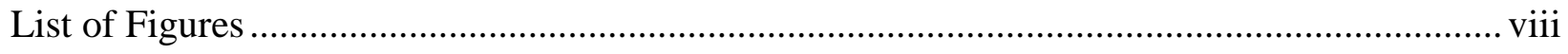

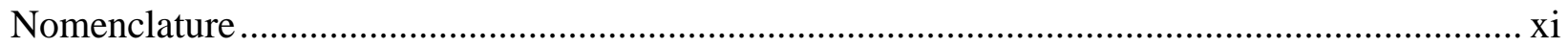

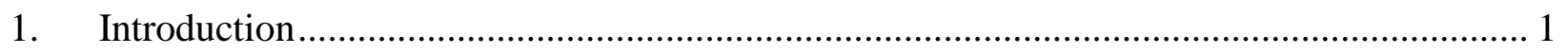

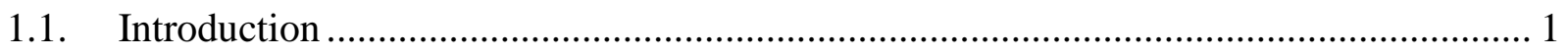

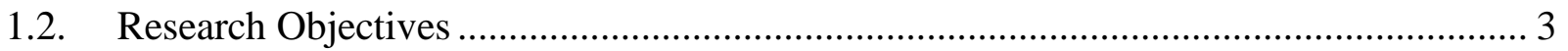

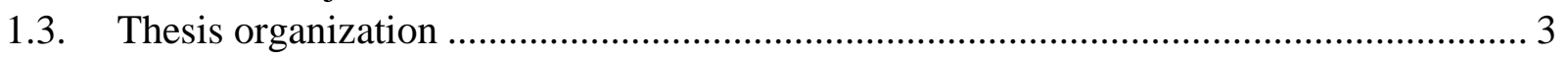

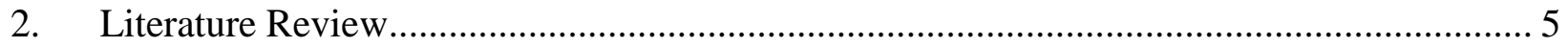

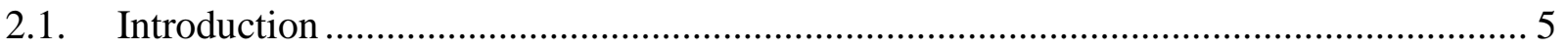

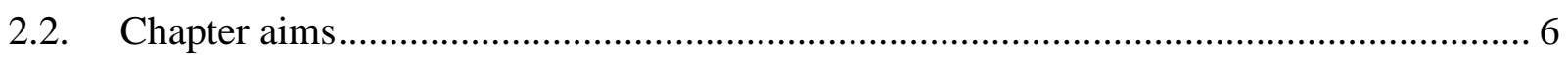

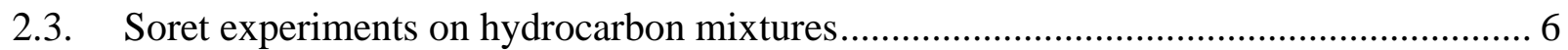

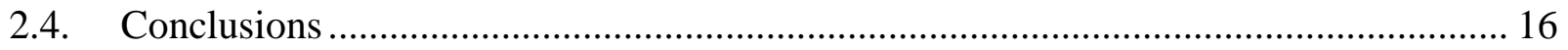

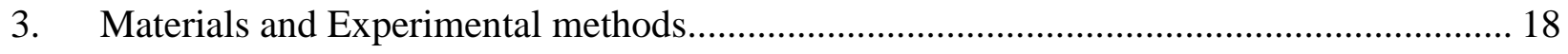

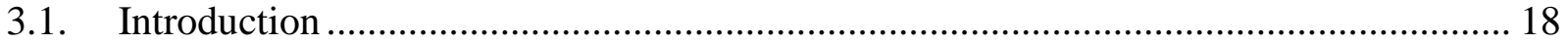

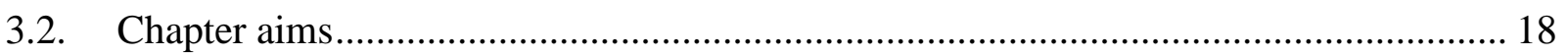

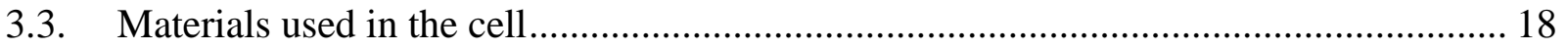

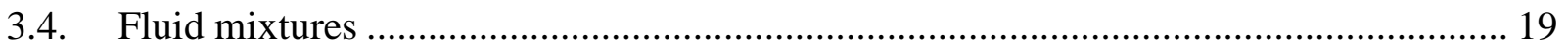

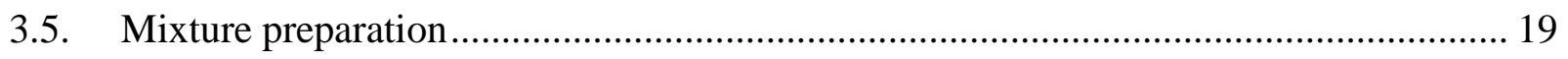

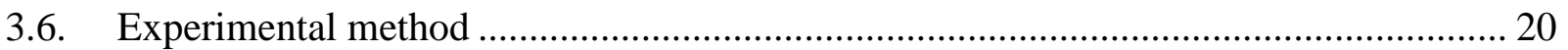

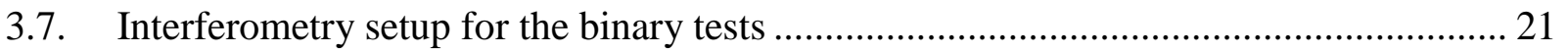

3.8. Interferometry setup for the ternary experiments.............................................. 26

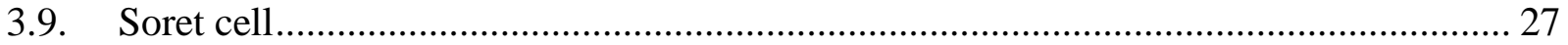

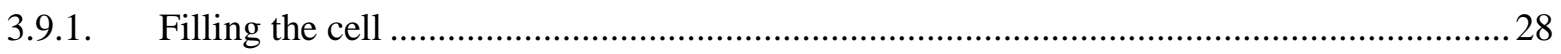

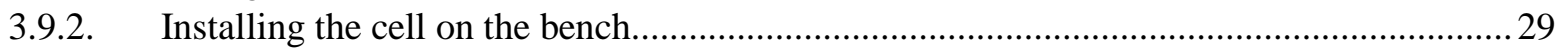

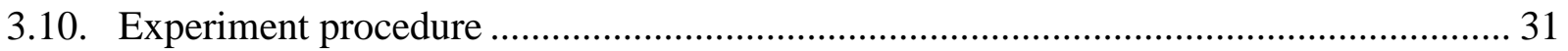

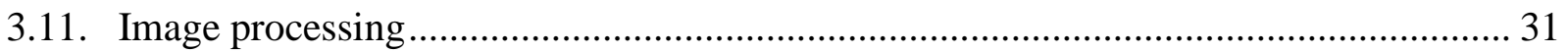

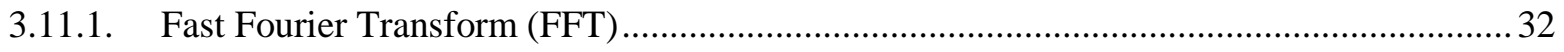

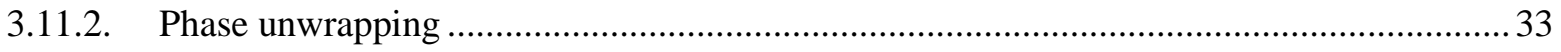

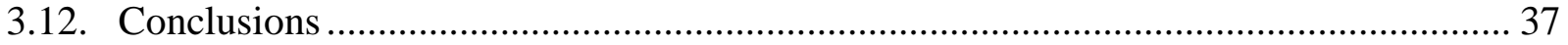

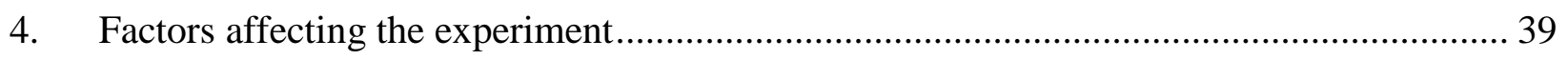

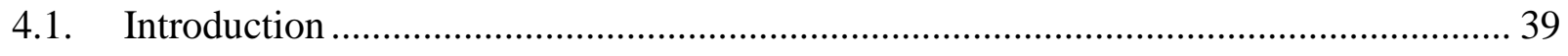

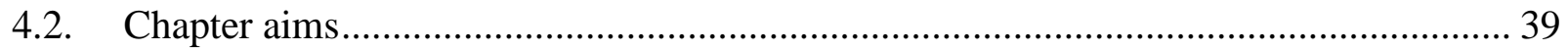




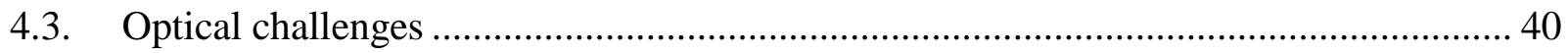

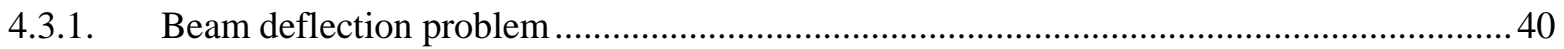

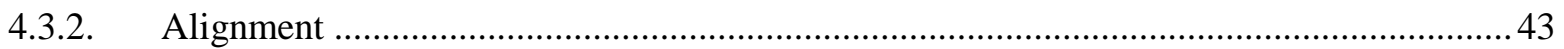

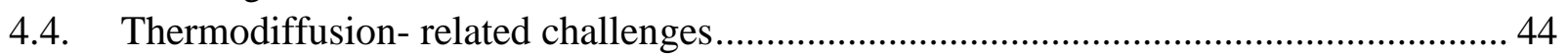

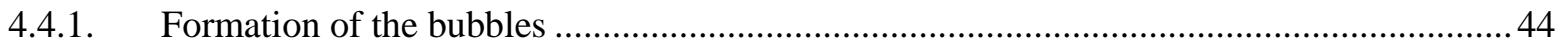

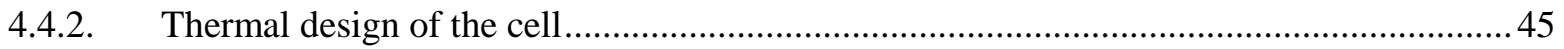

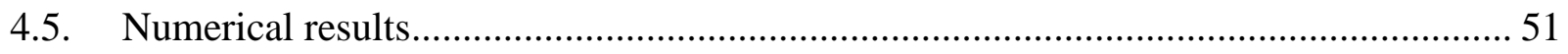

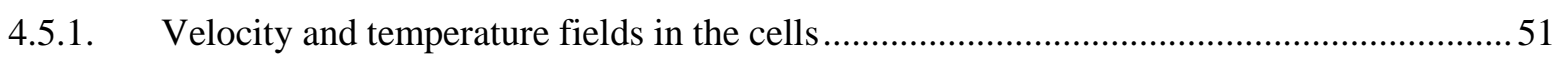

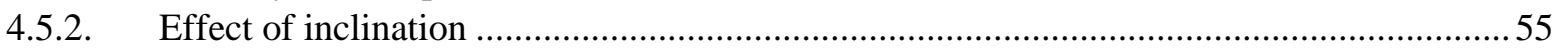

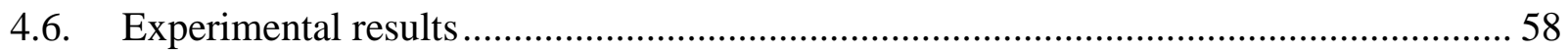

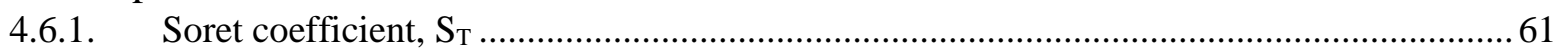

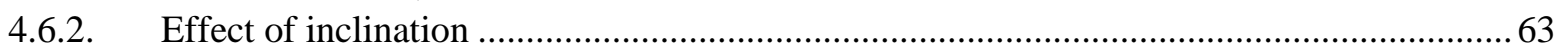

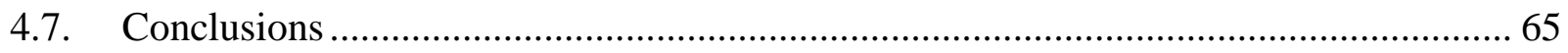

5. Data processing and error analysis for binary and ternary mixtures …………………........ 66

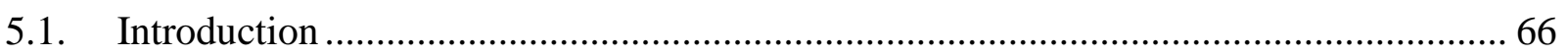

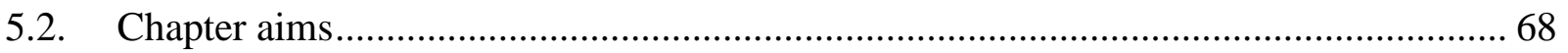

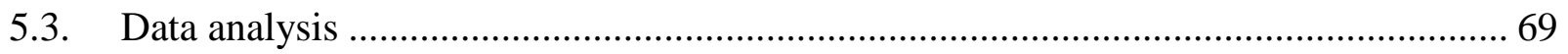

5.3.1. Subtraction of the reference image ………………................................................ 71

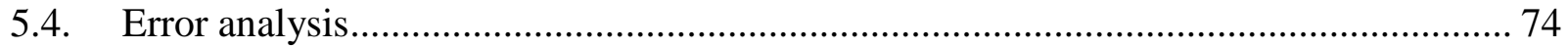

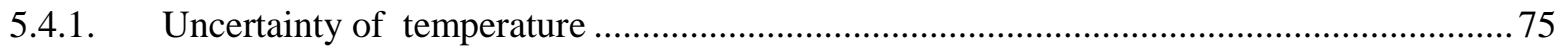

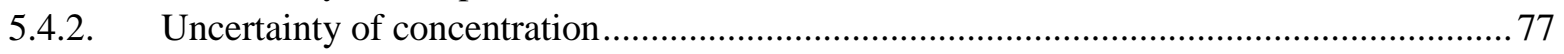

5.4.3. Uncertainty of the Soret coefficient ..............................................................................

5.4.4. Uncertainty in the concentration difference in ternary mixtures........................................79

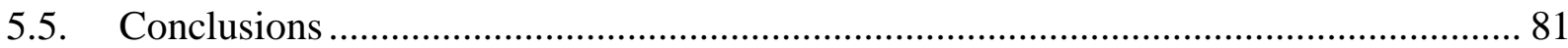

6. $\quad$ Measured soret coefficient for ternary mixtures ................................................................... 82

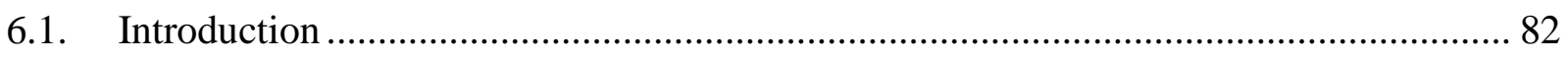

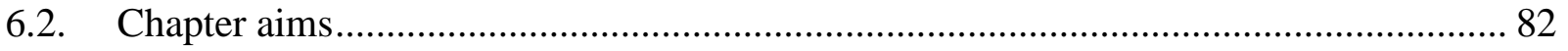

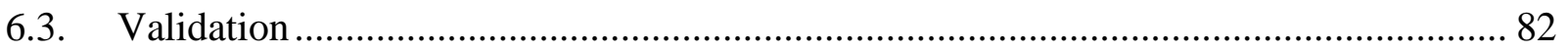

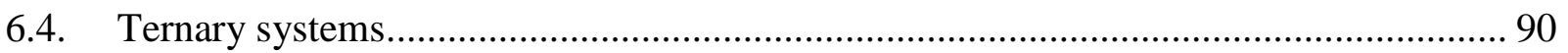

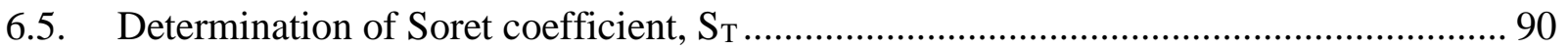

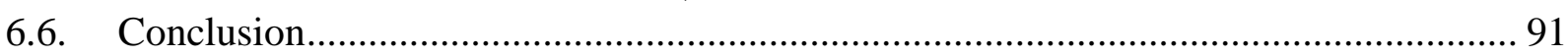

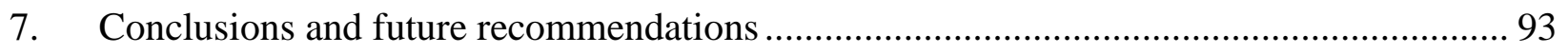

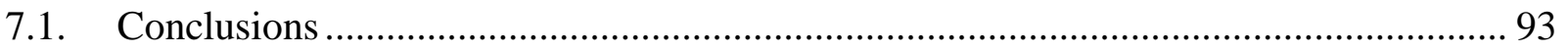

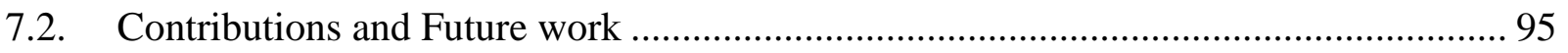

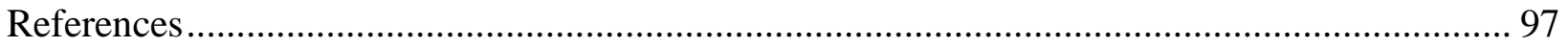




\section{List of Tables}

TABLE 2.1: SORET COEFFICIENTS FOR MASS FRACTION OF 0.50 AND TEMPERATURE OF $25^{\circ} \mathrm{C} \ldots \ldots . . . . . . . . . . . . .14$

TABLE 2.2: DCMIX1 BENCHMARK VALUES OF SORET COEFFICIENTS [75] ............................................ 16

TABLE 3.1: MASS FRACTION OF THE TERNARY MIXTURES USED IN THE PRESENT STUDY .......................... 19

TABLE 4.1: THERMAL PROPERTIES OF MATERIALS USED IN NUMERICAL MODELLING [83] ........................50

TABLE 4.2: MAXIMUM AND AVERAGE VELOCITIES IN THE MIXTURE (NUMERICALLY COMPUTED)..............52

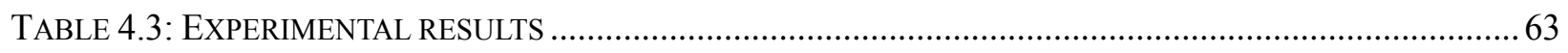

TABLE 5.1: REFRACTIVE INDEX OF SOME COMMON LIQUIDS AT $20^{\circ} \mathrm{C}$ AND WAVELENGTH OF 589.29 NM.. 69

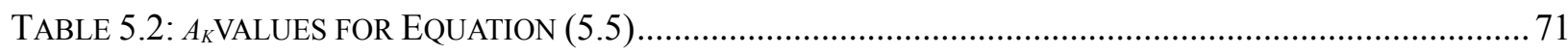

TABLE 5.3: MEASURED VALUES AND THEIR ESTIMATED UNCERTAINTIES ............................................... 74

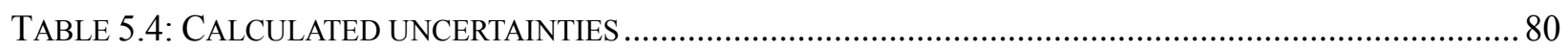

TABLE 6.1: MAXIMUM SEPARATION AND CORRESPONDING TEMPERATURE GRADIENT FOR DIFFERENT

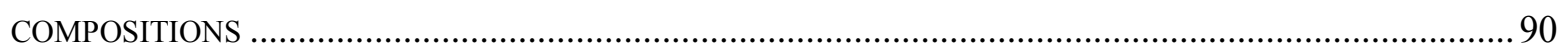

TABLE 6.2: MEASURED AND BENCHMARK VALUES OF SORET COEFFICIENT FOR 0.80/0.10/0.10 MIXTURE 91

TABLE 6.3: MEASURED SORET COEFFICIENT FOR THE NEW MIXTURES ...................................................91 


\section{List of Figures}

FIGURE 1.1: SKETCH OF A STANDARD SORET CELL USED IN CONVECTIONLESS TECHNIQUES.......................2

FIGURE 2.1: THERMOGRAVITATIONAL COLUMN AND THE SCHEME OF THE COLUMN [37].......................... 7

Figure 2.2: SEPARATION IN A THERMO GRAVITATIONAL COLUMN IN DIFFERENT ANGLES. SQUARES: EXPERIMENTAL DATA, SOLID LINES: THEORETICAL DATA [44] ....................................................... 8

FIGURE 2.3: FLOW CELL (TOP) AND BÉNARD CELL (BOTTOM) USED IN [50] .......................................... 9

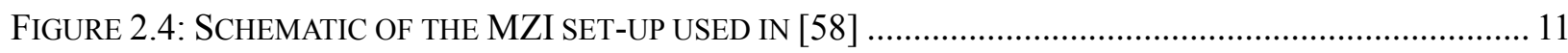

Figure 2.5: (A) TEMPERATURE AND (B) CONCENTRATION PROFILE OF A HYDROCARBON MIXTURE IN

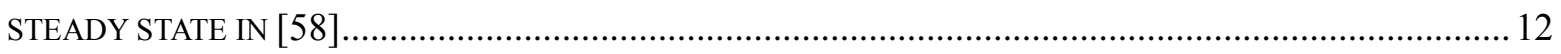

FIGURE 2.6: MOLECULAR STRUCTURE OF THE PURE COMPONENTS OF THE HYDROCARBON MIXTURE USED

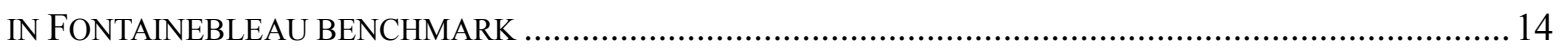

FIGURE 3.1: SCHEMATIC OF THE MACH-ZEHNDER INTERFEROMETER (MZI) ...........................................2 21

FIGURE 3.2: EFFECT OF SPATIAL FILTER ON THE QUALITY OF THE WAVEFRONT .........................................22

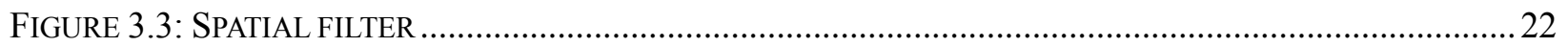

FigURE 3.4: COLLIMATING THE BEAM USING A PLANO-CONVEX LENS ....................................................23

Figure 3.5: TESTING THE COLLIMATION OF THE BEAM USING A SHEAR-PlATE COLLIMATION TESTER, SHOWING (A) COLLIMATED BEAM (B) NON-COLLIMATED BEAM ..................................................23

FIGURE 3.6: REDUCING THE ABERRATION BY CHOOSING THE CORRECT SIDE OF THE LENS TOWARDS THE

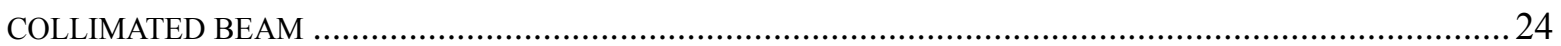

FiguRE 3.7: BEAM SPLITTER (A) PLATE BEAM SPLITTER (B) CUBE BEAM SPLITTER..................................25

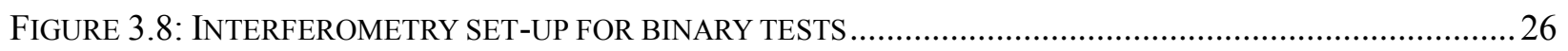

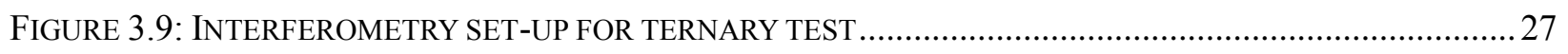

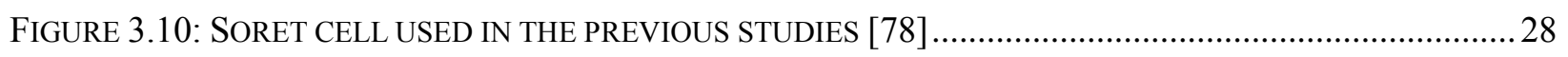

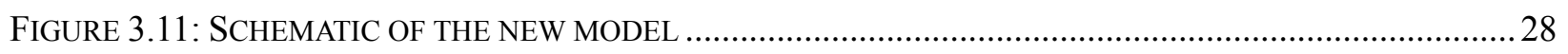

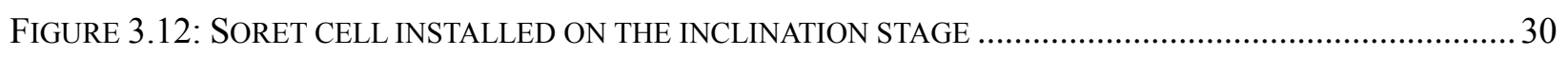

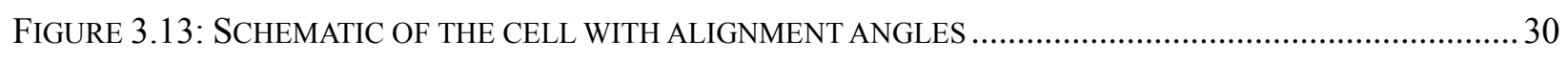

FIGURE 3.14: A TYPICAL FRINGE PATTERN CAPTURED FROM THE CELL IN THE CURRENT STUDY ................ 33

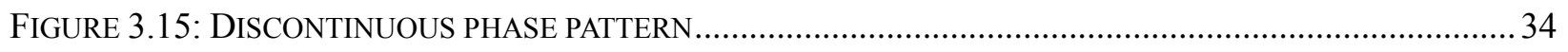

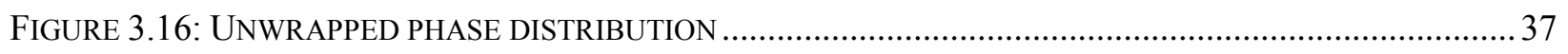

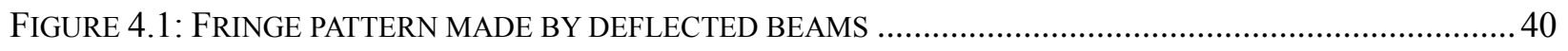

FIGURE 4.2: DISTANCE TRAVELLED AND OPTICAL PATH IN THE CELL WITH BEAM DEFLECTION ................. 41

FIGURE 4.3: DEFLECTED WAVEFRONT IN THE MEDIUM WITH A REFRACTIVE INDEX PROFILE .....................4 41

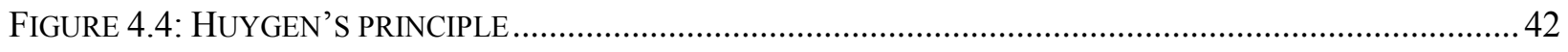


FIGURE 4.5: BEAM DEFLECTION IN A SORET CELL NEAR THE COLD SIDE .............................................. 43

FIGURE 4.6: LOW QUALITY FRINGES NEAR THE COLD SIDE CAUSED BY BEAM DEFLECTION ......................43

FIGURE 4.7: LOW QUALITY FRINGES NEAR THE COLD SIDE BECAUSE OF THE MISALIGNMENT ....................44

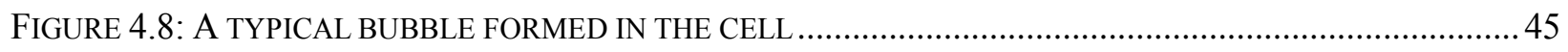

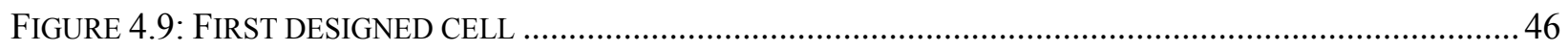

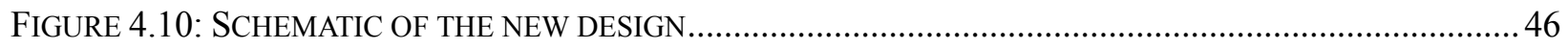

FIGURE 4.11 SCHEMATIC OF THE APPLIED BOUNDARY CONDITIONS FOR THE COMPUTATIONAL THERMAL

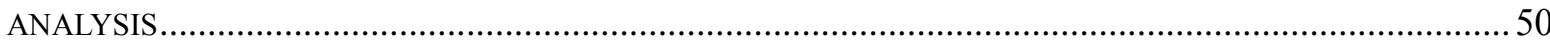

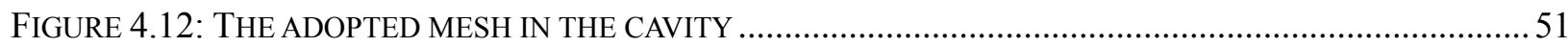

FIGURE 4.13: GRID SENSITIVITY TEST FOR BOTH MODELS BASED ON THE AVERAGE NUSSELT NUMBER .... 51

FIGURE 4.14: COMPUTED VELOCITIES IN DIFFERENT GEOMETRIES ........................................................52

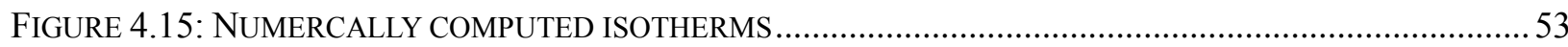

FIGURE 4.16: TEMPERATURE DISTRIBUTION ON A HORIZONTAL LINE NEAR THE HOT WALL ........................5 54

FIGURE 4.17: TEMPERATURE PROFILE ON A VERTICAL LINE BETWEEN HOT AND COLD SIDES OF THE CELL55

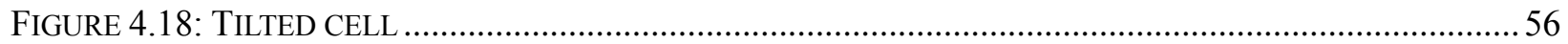

FIGURE 4.19: VELOCITY CONTOUR IN THE CELL WITH DIFFERENT INCLINATIONS (A)THETA=0, (B) THETA $=0.5$, (C) THETA=1, (D) THETA=2.5, (E) THETA=5 DEGREE ….............................................5 57

FIGURE 4.20: VELOCITY ON CENTER LINE FOR DIFFERENT INCLINATIONS OF THE CELL............................57

FIGURE 4.21: ISOTHERMS IN THE MIXTURE IN DIFFERENT INCLINATION................................................ 58

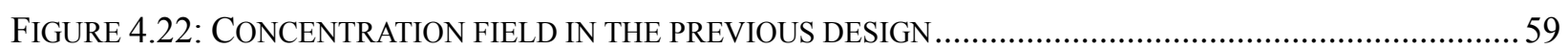

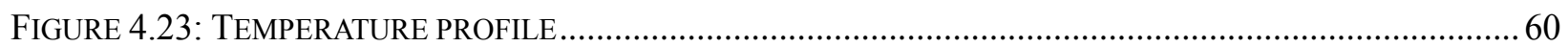

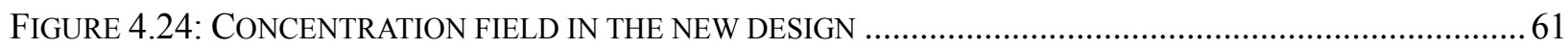

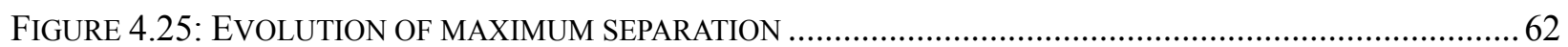

FIGURE 4.26: CONCENTRATION CONTOUR AT DIFFERENT INCLINATIONS OF THE CELL ...............................64

FIGURE 4.27: CONCENTRATION PROFILE AT DIFFERENT INCLINATIONS OF THE CELL ................................64

FIGURE 5.1: FRINGE PATTERN IN THE MIXTURE ESTABLISHED BECAUSE OF OPTICAL PATH DIFFERENCE BETWEEN MACH-ZEHNDER ARMS: (A) NO TEMPERATURE GRADIENT BETWEEN THE SIDES, (B) 2.5 DEGREE CELSIUS DIFFERENCE BETWEEN THE TOP AND COLD SIDE............................................67

FIGURE 5.2: EXTRACTING INFORMATION FOR THE FRINGE PATTERNS USING FFT METHOD ...................... 68

FIGURE 5.3: TEMPERATURE DISTRIBUTION ALONG THE VERTICAL LINE IN THE CENTRE OF THE CELL WITH

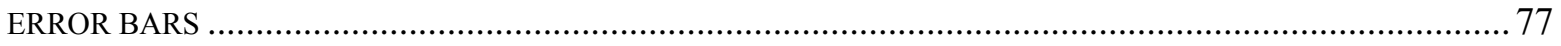

FIGURE 5.4: THE EVOLUTION OF THE CONCENTRATION DIFFERENCE BETWEEN THE HOT AND COLD SIDES

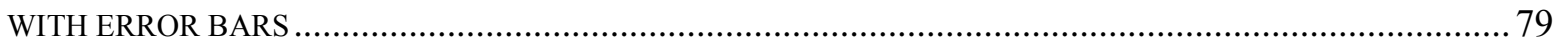


FIGURE 6.1: FRINGE PATTERN OF A BINARY MIXTURE UNDER (A) ISOTHERMAL CONDITIONS (B) WITH A

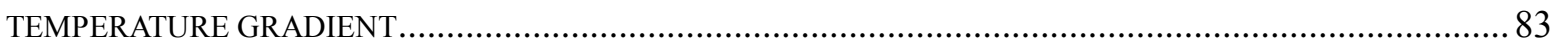

FIGURE 6.2: UNWRAPPED PHASE BAND INCLUDING INFORMATION ABOUT TEMPERATURE AND

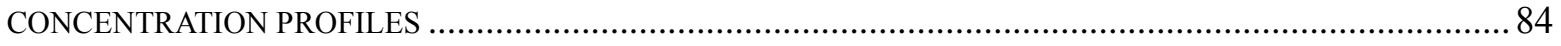

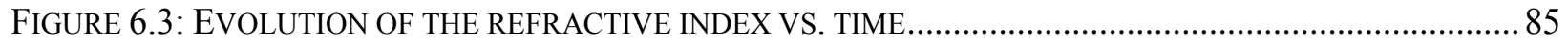

FIGURE 6.4: TEMPERATURE DISTRIBUTION OBTAINED EXPERIMENTALLY AT (A) $\mathrm{T}=0$ AND (B) $\mathrm{T}=7$ MINUTES .85

FIGURE 6.5: TEMPERATURE PROFILE FROM THE COLD SIDE TO THE HOT SIDE …..................................... 86

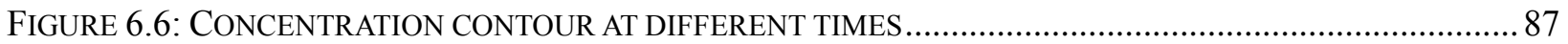

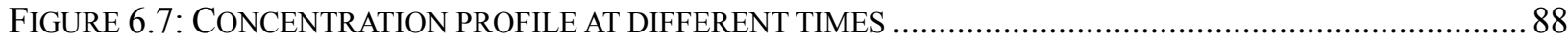

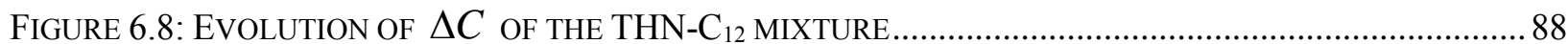

FIGURE 6.9: EVOLUTION OF THE CONCENTRATION DIFFERENCE BETWEEN HOT AND COLD WALLS (A) RESULT

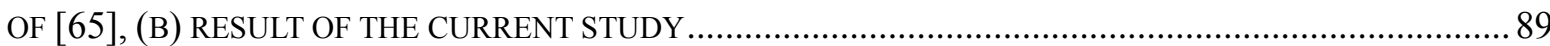




\title{
Nomenclature
}

\author{
Alphabetical \\ $c_{i} \quad$ mass fraction of mixture component $i$ \\ $c_{0}$ initial mass fraction \\ $C_{p} \quad$ specific heat capacity $\left(\mathrm{J} \mathrm{g}^{-1} \mathrm{k}^{-1}\right)$ \\ $D_{M}$ isothermal molecular diffusivity $\left(\mathrm{cm}^{2} \mathrm{~s}^{-1}\right)$ \\ $D_{T}$ thermodiffusion coefficient $\left(\mathrm{cm}^{2} \mathrm{~s}^{-1} \mathrm{k}^{-1}\right)$ \\ $g$ gravitational acceleration $\left(\mathrm{cm} \mathrm{s}^{-2}\right)$ \\ $h$ height of the cell (mm) \\ $J_{i}$ molar flux of mixture component $i\left(\mathrm{~J} \mathrm{~cm}^{2} \mathrm{~cm}^{-1}\right)$ \\ $k$ thermal conductivity $\left(\mathrm{J} \mathrm{s}^{-1} \mathrm{~cm}^{-1} \mathrm{k}^{-1}\right)$ \\ $n$ index of refraction (-) \\ $P$ pressure $(\mathrm{Pa})$ \\ $P_{\text {atm }}$ atmosphere pressure $(\mathrm{Pa})$ \\ $S_{T}$ Soret coefficient $\left(\mathrm{K}^{-1}\right)$ \\ $T$ temperature (K) \\ $T_{a m b}$ ambient temperature $(\mathrm{K})$ \\ $u \quad$ velocity component in $x$ direction $\left(\mathrm{cm} \mathrm{s}^{-1}\right)$ \\ $v$ velocity component in $y$ direction $\left(\mathrm{cm} \mathrm{s}^{-1}\right)$ \\ Greek \\ $\mu$ dynamic viscosity (Pa. s) \\ $\rho$ density $\left(\mathrm{g} \mathrm{cm}^{-3}\right)$ \\ $\tau_{t h} \quad$ thermal characteristic time (s) \\ $\tau_{D}$ diffusion characteristic time (s) \\ $\theta$ inclination angle $\left(^{\circ}\right)$ \\ $\alpha$ Thermal diffusivity \\ $\beta$ Thermal expansion coefficient $\left(\mathrm{K}^{-1}\right)$ \\ $v \quad$ Kinematic viscosity $\left(\mathrm{m}^{2} \mathrm{~s}^{-1}\right)$
}





\section{INTRODUCTION}

\subsection{INTRODUCTION}

Mass diffusion in isothermal multicomponent mixtures can occur due to the presence of the concentration gradient. However, by applying temperature gradient, diffusion takes place even in homogenous mixtures with no concentration gradient. This kind of diffusion is called thermodiffusion. Although the same phenomenon had already been discovered and described by a German scientist named Ludwig [1], Soret [2] later studied this effect in more detail and the phenomenon is now named after him. This phenomenon is also known as thermal diffusion, the Ludwig-Soret effect, or the Soret effect.

One of the most important applications of thermal diffusion is in the oil industry where the components of hydrocarbon compositions can be separated by applying a temperature gradient, causing mass diffusion from the Soret effect [3]. Components of hydrocarbon mixtures in oil reservoirs with almost critical temperature and pressure can be separated due to this thermal diffusion effect [4]. Using values of the concentration differences and temperature gradients in a mixture, the Soret coefficient is calculated, and this coefficient is used to express the thermal diffusion in the system. A schematic of a standard Soret cell for convectionless measurement of concentration field is shown in Figure 1.1. In this Soret cell, a rectangular cavity is filled by a multicomponent mixture, the side walls are adiabatic, and the entire system is at a mean temperature of $\mathrm{T}_{0}$. At a given time, a temperature gradient is applied in the vertical direction. The top and bottom surfaces play the role of the hot and cold sides, respectively. Diffusive flux in a multicomponent system can be induced by a concentration gradient, thermal diffusion, or a pressure gradient. In the above system, it is assumed that the pressure gradient is negligible because of the sufficiently small height of the experimental setup. Thus, the pressure gradient is ignored. 


\begin{tabular}{|l|}
\hline Hot Side \\
\hline Mixture \\
\hline Cold Side \\
\hline
\end{tabular}

\section{Figure 1.1: Sketch of a standard Soret cell used in convectionless techniques}

The separation of components starts with the application of the temperature gradient. The mathematical model describing the process must include coefficients of both mass diffusion (concentration based) and thermal diffusion. The diffusive flux of component $i$ in a multicomponent mixture with $N$ components is calculated as below[5]:

$$
\begin{gathered}
J_{i}=-\rho\left(\sum_{k=1}^{N-1} D_{i k} \nabla c_{k}+D_{T, i} \nabla T\right) \quad i=1,2, \ldots, N \\
\sum_{i=1}^{N} c_{i}=1
\end{gathered}
$$

Where $c_{i}$ is the mass fraction of component $i, \rho$ stands for the density, $D_{i k}$ and $D_{T i}$ are Fick's mass diffusion and thermal diffusion coefficients, respectively, $\nabla c$ represents the gradient of mass fraction induced by thermal diffusion due to the temperature gradient $\nabla T$. There are $\mathrm{N}-1$ independent mass fluxes. In literature, there is not agreement about the sign of the second term on the right hand of Equation (1.1), and some authors choose to give it a negative sign. For binary mixtures, Equation (1.1) for component 1 is simplified as follows:

$$
J_{1}=-\rho\left(D \nabla c_{1}+D_{T} c_{0}\left(1-c_{0}\right) \nabla T\right)
$$

Thermal diffusion is shown with $D_{T} c_{0}\left(1-c_{0}\right)$, where $c_{0}$ is the initial value of the mass fraction. Obviously the thermodiffusion effect does not happen in pure fluids. In binary mixtures the mass flux is zero $(J=0)$ when the system reaches a steady-state condition. Then Equation (1.3) can be shown as:

$$
\Delta c_{1}=-\frac{D_{T}}{D} c_{10}\left(1-c_{10}\right) \Delta T
$$


$S_{T}$ is called the Soret coefficient and is equal to the ratio of $D_{T}$ over $D$, which is obtained from Equation (1.5).

$$
S_{T}=\frac{-1}{c_{10}\left(1-c_{10}\right)} \frac{\Delta c_{1}}{\Delta T}
$$

By measuring the concentration difference and the corresponding temperature gradient, the Soret coefficient is found from Equation (1.5). The calculated $S_{T}$ contains information about the diffusion behaviour of the components. In binary mixtures, the negative value of the thermal diffusion coefficient or Soret coefficient shows that the chosen reference component $i$ migrates towards the hot side of the cell while in ternary and higher mixtures the direction of the component migration depends on the values of both the cross diffusion and thermal diffusion coefficients. The molecules that travel toward hot side are called thermophilic and the ones that migrate towards the cold side are named thermophobic [6].

\subsection{RESEARCH OBJECTIVES}

The main goal of this thesis was to obtain Soret coefficients of binary and ternary hydrocarbon mixtures experimentally by using interferometry setup with Mach-Zehnder arrangement. To achieve this goal, thermal analysis was done numerically to re-design and optimized the Soret cell used in space experiments. In order to improve the Soret coefficient measurement and uncertainty reduction, the geometry of the cell was modified and it was prepared

for ground-based experiments so the expensive experiments in space can be performed on the Earth with cheaper costs. In this dissertation, previous cell means the cell used in space experiments. In addition, for the first time, the effect of the inclination of the cell on thermal diffusion was investigated numerically and experimentally. Finally, the Soret coefficients of ternary hydrocarbon mixtures with five different mass fractions were measured experimentally and new values were proposed to establish a benchmark.

\subsection{THESIS ORGANIZATION}

In the first chapter, the introduction of the thesis is presented and the general idea about the extraction of Soret coefficient in convection free experiments is presented. In the second chapter, the previous studies in field of thermal diffusion are reviewed and different experimental methods 
to measure the concentration and thermal diffusion are explained and finally the importance of the present study is stated. Chapter three gives details about the experimental technique used in this study. The optical interferometry as the measurement instrument is explained in details. Two different geometries of the Soret cell are explained and compared to each other and the experimental procedure is presented. Chapter four describes the factors that affect the optical measurement and also thermal diffusion process. The numerical analysis in order to study heat transfer in the Soret cell is described. Chapter five deals with the image processing of the captured fringe patterns from the interferometry and extracting the information from the images. The error analyses is also included in this chapter. Chapter six presents the experimental results for ternary mixtures and the validation of the results is discussed. Finally, in chapter seven, conclusions are given. Contribution and future works are also presented. 


\section{LITERATURE REVIEW}

\subsection{INTRODUCTION}

In 1879 [7], Charles Soret published the first paper discussing the thermal diffusion effect. He experimentally found that a uniform composition of a salt solution in a two ended tube exposed to a temperature gradient does not remain uniform, and because of the temperature difference between the ends of the tube the salt moves towards the cooled end [2]. He performed the experiments in two differently shaped tubes: a straight tube and a U-shape tube. One end of the tubes was placed in a hot chamber $\left(78^{\circ} \mathrm{C}\right)$ with circulating hot water and the other end was in cold water with a temperature of $15-18{ }^{\circ} \mathrm{C}$. The solutions used were $\mathrm{NaCl}$ and $\mathrm{KNO}_{3}$ in water. The length of the tubes were $30 \mathrm{~cm}$ in each experiment, and a typical experiment took 10-25 days. After the temperature gradient was held for the experiment length, samples were taken from the upper, the middle, and the lower zones of the tube and the concentrations of salt in each zone were measured. His results were in agreement with the results obtained by C. Ludwig [1] more than 20 years before. In all likelihood, however, Soret was probably not aware of Ludwig's work.

The order of the absolute value of the Soret coefficient in a liquid composition is $\left|S_{T}\right| \sim 10^{-3}-10^{-2} K^{-1}$, and for a binary mixture with an initial mass fraction of $50 \%$ for the reference component with a $4{ }^{\circ} \mathrm{C}$ temperature gradient, the order of the concentration difference is the same as $\mathrm{S}_{\mathrm{T}}$. Although this value seems small, the effect at this magnitude is very important and has many applications: For example, some natural activities such as convection in stars [8] and the physics of solar ponds [9] are affected by the Soret effect. The temperature gradient in the ocean leads to thermal diffusion and a salinity gradient that is called thermohaline convection [10]. Separation and characterization of polymers occurs mainly due to the thermodiffusion effect [1114]. Feld-flow-fractionation devices work based on this phenomenon [15-20], and they are used in the separation of bacteria [21, 22], colloidal materials [23, 24], charged particles [25], and others. Some studies have been done on the effect of thermodiffusion on the freezing processes of foods[26]. Bonner and Sundelof [27] suggested thermodiffusion as a mechanism of biological 
transport. The determination of biomolecular binding curves [28] and trapping of DNA [29] are other examples of biological applications of thermal diffusion.

\subsection{CHAPTER AIMS}

The experimental methods to measure thermal diffusion in mixtures are reviewed in the present chapter and the advantages and drawbacks of each method is explained. Moreover, the established thermal diffusion benchmark of DCMIX1 for hydrocarbon mixtures is introduced.

\subsection{SORET EXPERIMENTS ON HYDROCARBON MIXTURES}

One of the most important applications is in the oil industry where the components of hydrocarbon compositions can be separated by applying temperature gradients and consequently using the Soret effect [30]. Some studies using thermogravitational columns were done by a research group to investigate and prove the effectiveness of the Soret effect in oil reservoirs [3134]. A sample of two different oil fields were used and it was found that because of temperature gradients, components were separated, and then due to the buoyancy force the lighter components moved to the top of the mixture.

Scientists have been studying thermal diffusion in multicomponent mixtures with different techniques for several years. The very first step of studying this phenomenon is to study the multicomponent mixtures with the minimum number of components. So binary mixtures are the first candidates to be tested and an adequate measurement technique must be established to study Soret effect in such compositions. Several different methods with different accuracies and applicability have been proposed. Basically, experimental techniques to measure Soret coefficients can be categorized into two main groups [35]: (a) convective coupling methods and (b) convectionless methods.

In terrestrial condition because of the gravity force, natural convection in a system being heated from one side is unavoidable. This sort of convection in a test setup for thermal diffusion experiments can mixing back the mixture and prevents the separation of the components. In 1938 a setup was introduced by Clusius and Dickel [36] that takes advantage of the gravity in segregation of the components in a multicomponent mixture. This set up is named thermodiffusion

column or thermogravitational column (TGC) or Clusius-Dickel column. The general view of a thermogravitational column is depicted in Figure 2.1. The original column consisted of two 
concentric cylinders. The temperature of the inner cylinder was more than the outer one and a gaseous mixture was trapped between the two cylinders. Because of thermal diffusion effect some components move towards the hot side and some move towards to the cold side. Then the lighter components float on top of the heavier ones and a concentration gradient will be established between top and bottom of the column.
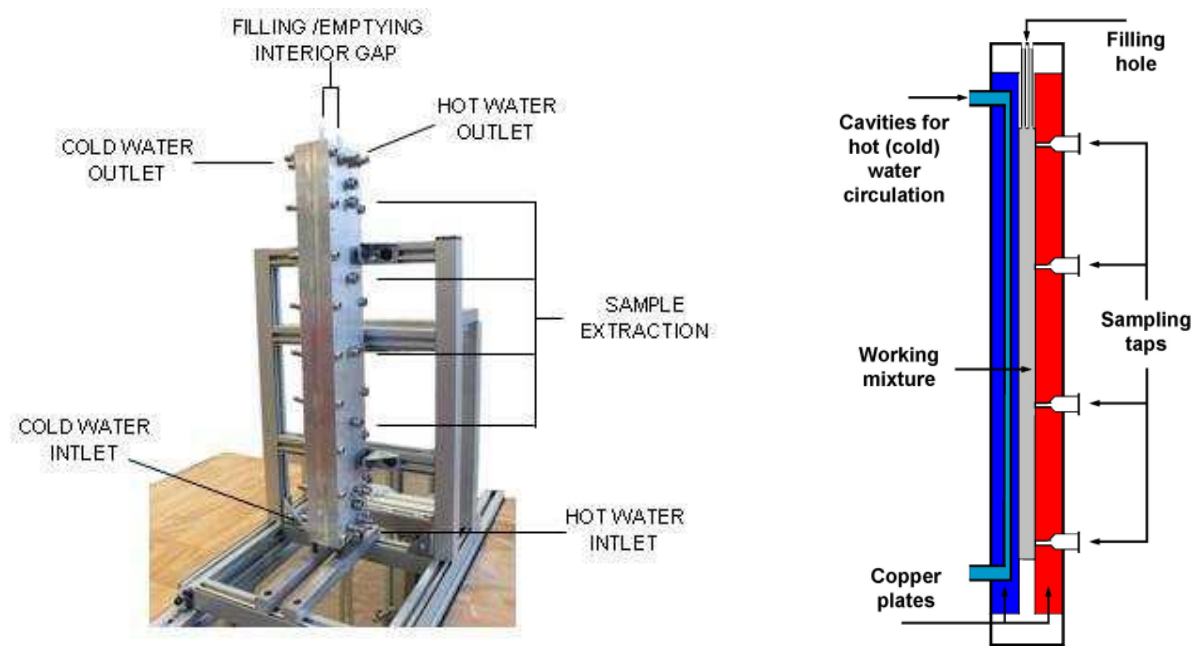

Figure 2.1: thermogravitational column and the scheme of the column [37]

This first thermogravitational column was designed for gaseous mixtures. Later on more modifications were applied to it and it was used for liquid binary mixtures [38, 39]. This technique was used in hydrocarbon mixtures for the first time by Danby et al. [40]. Dutrieux et al. [41] found Soret coefficient of water-ethanol system using thermogravitational column. More recently, Blanco et al. [42] used this technique to measure Soret coefficient of binary hydrocarbon mixtures. Costeseque [43] studied thermal diffusion effect in binary hydrocarbon mixtures placed in a thermogravitational column packed with porous media. In an innovative investigation by Platten et al. [44] effect of the inclination of the column on the separation of components was studied and it was shown that by tilting the column the separation increases significantly. The tested mixture was water-ethanol. For larger separation, they suggested using longer columns, higher temperature difference, and inclination close to an optimal angle. The experimental results are in a good agreement with their data obtained from theory as it is shown in Figure 2.2. 


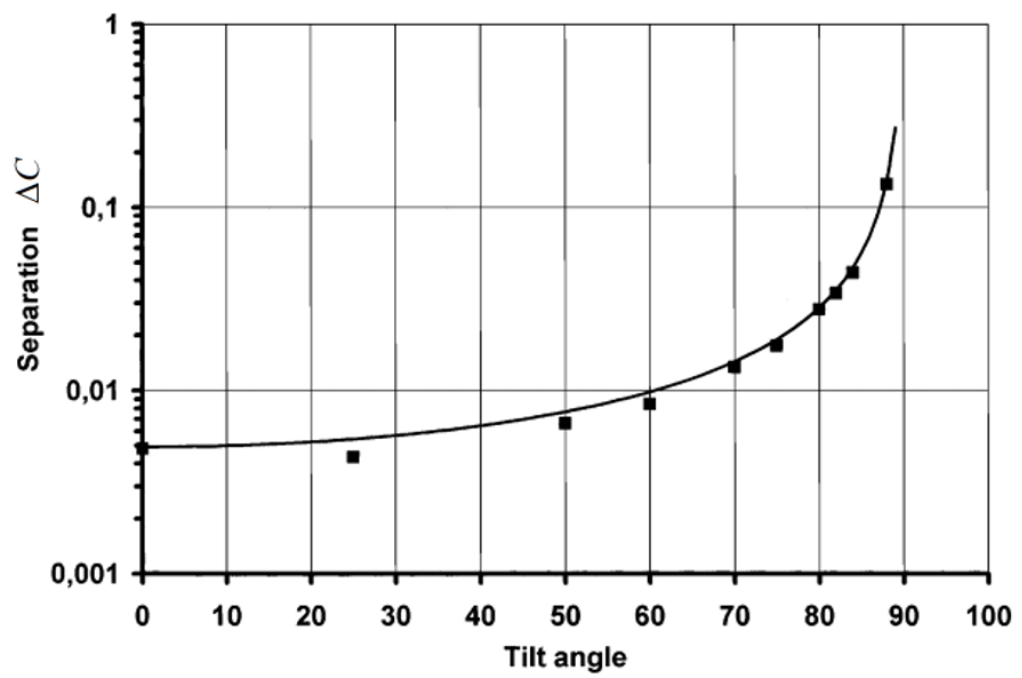

Figure 2.2: Separation in a thermo gravitational column in different angles. Squares: experimental data, Solid lines: theoretical data [44]

As can be seen in Figure 2.2, at a 25 degree inclination the separation is less than that in vertical column (tilt angle $=0$ ), and this was not explained by the authors. After this abnormal point the separation starts rising slightly, and between 80 and 90 degrees there is a sudden increase in the segregation.

Saxton et al. [45] designed a two-cell system to perform thermal diffusion measurements. A membrane made of an ultrafine porosity fritted glass was used to separate the cells and two stirrers were used to make the composition uniform in each cell. The samples were collected form the cells separately and analysed in a refractometer. The same results were found for heating from the bottom and the top of the system and a certain porosity value was introduced for which a threshold of the stirrer speed was established, above which the speed of stirring has no effect on the separation.

In 1962, Turner [46] used a flow cell to run thermal diffusion experiments on aqueous solutions. The cell was made of two horizontal plates while the upper and lower plates play the role of the hot and cold sides, respectively. Laminar flow of a homogenized mixture passes between the plates, and separation occurs due to the temperature gradient between the plates. The first designed cell was not accurate for slow flow rates. Later on, Turner et al. [47-49] improved the design. The new cell was calibrated with aqueous cadmium sulphate that has a known thermal diffusion coefficient. The problem of the previous design was the undesirable convections at low 
flow rates, which could mix the solution. In the new design, however, the convection effects were negligible and therefore it was applicable for a wide range of flow rates. After the calibration, the cell was used for organic solutions.

In convection-free apparatuses, convective disturbances must be avoided. In 1997, Vaernbergh and Legros [50] used both the flow cell and a Bénard cell with large aspect ratios (width/height) in order to overcome the problem of convective disturbances. Figure 2.3 illustrates the cells used in their work. The Bénard cell consists of two horizontal plates with a vertical gap of $d$ in between. The gap is filled with the solution, which is heated from below.
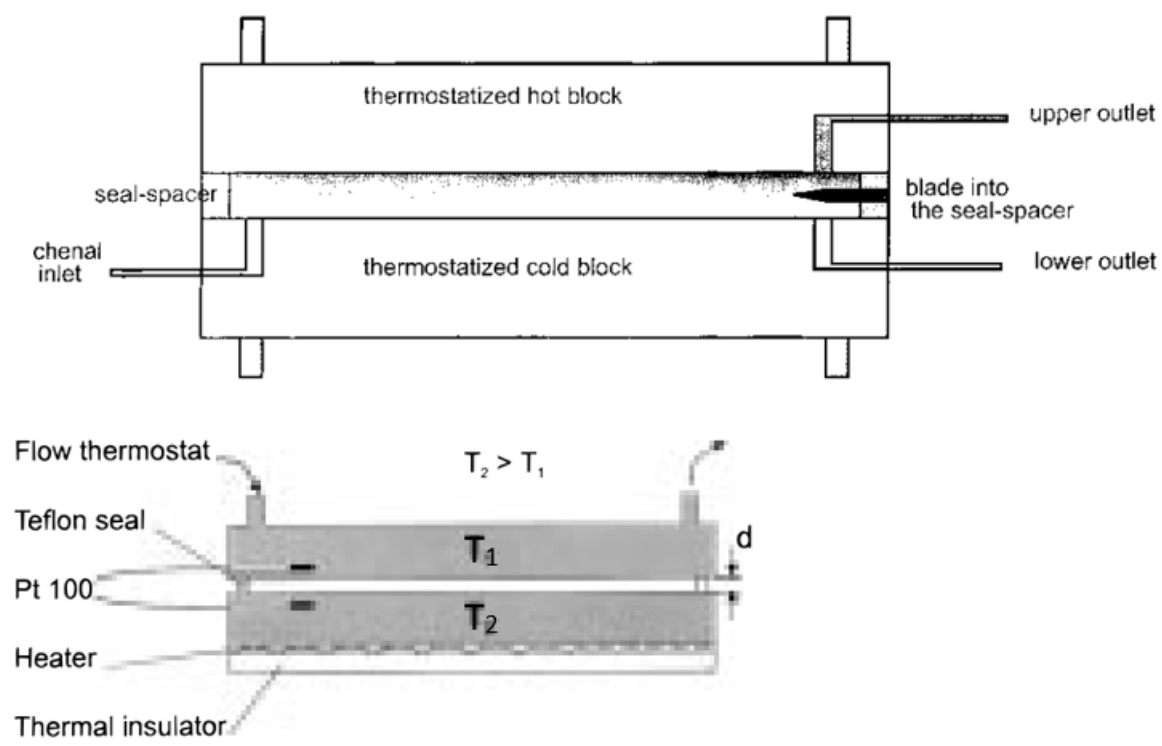

Figure 2.3: Flow cell (top) and Bénard cell (bottom) used in [50]

The onset of the convection in a liquid heated from below is estimated by the critical Rayleigh number [51]:

$$
R a_{c}=\frac{g \beta \Delta T}{v \alpha} h^{3}=1707.8
$$

Where $g$ is the gravity, $\beta$ is the thermal expansion coefficient, $\alpha$ is thermal diffusivity, $v$ is kinematic viscosity, and $h$ represents the height of the cell. The threshold of convection in a Bénard cell filled with a solution depends on the sign of the corresponding Soret coefficient. In a mixture with a positive $S_{T}$, the denser component moves towards the cold side and convection commences with a lower temperature gradient, while in the case of a negative $S_{T}$, where the denser 
component migrates towards the hot side, the convection flow starts with a higher temperature difference [50]. Therefore, the Bénard cell is only used for mixtures with negative Soret coefficients, because of better stability.

Giglio and Vendramini [52] introduced a method of using the thermal lens effect in a binary mixture to build up a thermally induced concentration gradient (Soret effect).

The same research group studied thermal diffusion in a classical Soret cell using a beam deflection technique (BDT) for the first time [53]. In a BDT set up, the side walls are transparent to the laser beams. Deflection of the beam due to the concentration and temperature gradients takes place while it passes through the cell $[35,54-56]$. A CCD camera is used to detect and image the deflected beams, and by processing the captured images, the concentration gradient is measured.

Thermal diffusion forced Rayleigh scattering (TDFRS) [57] is an optical grating technique in the category of the convectionless systems. In this technique, a beam from a laser source is split into two beams with equal intensity. Then, the beams intersect and are focused by a lens onto the sample. As a result, a holographic interference grating is established. Thanks to an inert dye added to the sample, the energy of the light is absorbed and raises the temperature. The established periodic temperature profile induces a periodic concentration profile because of the Soret effect. A third laser beam reads out this refraction grating, which is created by the temperature and concentration profiles. Two contrast factors, $\partial n / \partial T$ and $\partial n / \partial C$, are used to find the temperature and concentration profiles. The small characteristic length in the TDFRS technique, approximately $10 \mu \mathrm{m}$, leads to a short relaxation time. However, the experiments must be carried out several times, and the final result is the average of the values obtained in each test.

Shevtsova and Mialdun [58] developed a method of optical digital interferometry (ODI) to study thermal diffusion in binary hydrocarbon mixtures. Because of being non-intrusive, ODI is one of the most popular methods used to measure Soret coefficients nowadays. This method is based on the variation of the index of refraction of the sample in a Soret cell. The schematic of the standard Soret cell used in an ODI technique was shown previously in Figure 1.1. The main characteristic of the cell used in [58] was similar to the standard Soret cell. The mixture is within the cell surrounded by transparent lateral walls and sandwiched between two copper blocks with constant temperatures. It was the first report on a method that allows obtaining the temperature and concentration fields in the mixture entirely at different times of the experiment transiently. The 
transparent cubic cell is initially filled by a homogeneous mixture, and then, by applying a thermal gradient between the blocks, the thermal diffusion step starts. Figure 2.4 illustrates the MachZehnder interferometry (MZI) arrangement utilized in [58].

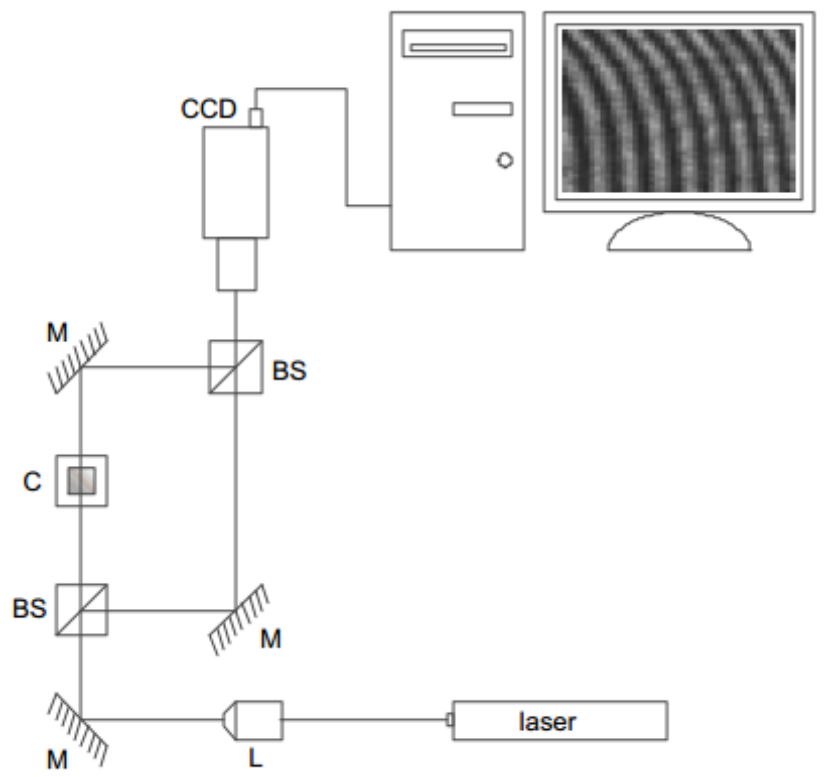

Figure 2.4: Schematic of the MZI set-up used in [58]

A spatial-filter expands the light beam produced by the laser source, and a plano-convex lens collimates the expanded beams. Thereafter, the collimated beam passes through a beam splitter and is divided into two beams of equal intensity. The Soret cell is installed in one arm of the Mach-Zehnder setup while in the second arm the beam passes through an undisturbed environment. The refractive index of the mixture changes due to the variation of the temperature and the concentration. As a result, the wave-front of the laser beam is modulated. The disturbed and undisturbed beams will be recombined and the final wavefront is imaged by a CCD camera. By using a 2D fast Fourier transform (FFT) the phase bands of the interferogram are obtained and the refractive index variation is calculated from the phase distribution:

$$
\Delta n(x, z, t)=\frac{\lambda}{2 \pi L} \Delta \varphi
$$

where $n$ is refractive index, $\lambda$ is the wavelength $(632.8 \mathrm{~nm})$, and $\varphi$ is the phase.

Finally, the temperature and concentration distributions are calculated from the refractive index distribution. 
The applied temperature gradient in this work was $10{ }^{\circ} \mathrm{C}$ and $5{ }^{\circ} \mathrm{C}$ when the mean temperature was $25^{\circ} \mathrm{C}$. They found that the temperature profile along the vertical line in the center of the cell was almost linear at steady state. However, small non-linearities were observed near the top and bottom sides. The concentration profile was also linear in the central profile. Moving away from the central part, to the top and bottom sides, however, the linearity decreases (Figure 2.5). It was proposed that two effects could cause this problem: first, residual convection due to a horizontal temperature gradient, and second, the presence of bubbles. Based on the temperature profile along the vertical centerline, the cell could be divided into three regions. The strongest convection happened in the top region of the cell, and the concentration in this region was almost independent of the height. In the lower region of the cell, although the corner convection was reduced, it still disturbed the mixture. The central region of the cell was essentially free from convection. Separation of components in this zone happens due to the Soret effect. It was discovered that convective flows do not appear in this zone and consequently, it is meaningful to measure the transport coefficients in the middle of the cell using the concentration and temperature profiles.

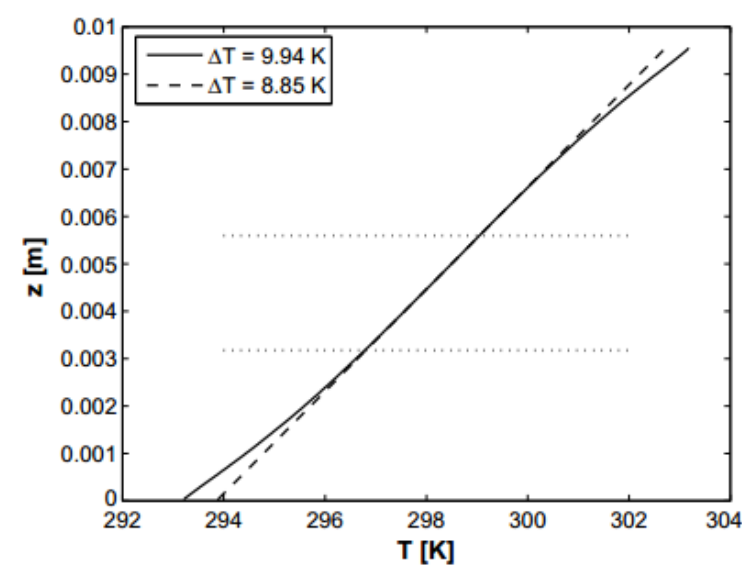

(a)

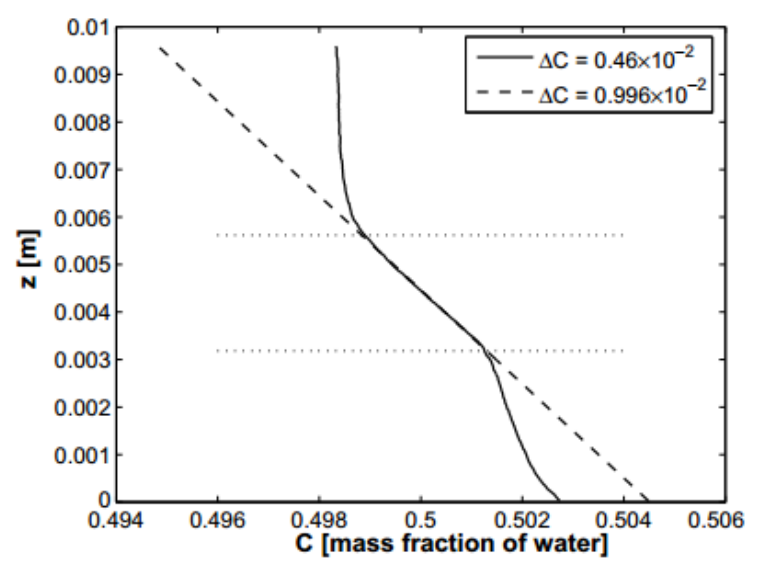

(b)

Figure 2.5: (a) Temperature and (b) concentration profile of a hydrocarbon mixture in steady state in [58] 
The separation continues until the concentration reaches a steady state. At this moment, $\Delta c_{\max }$ at the convection-free zone (linear temperature and concentration profiles) and the corresponding $\Delta T$ are used to measure the Soret coefficient.

Also, it was found in that study that the convection induced in the corners of the cell was due to horizontal gradients and that the main reason for the existence of horizontal temperature gradients is the poor heat transfer through the seals at the interface of the liquid, quartz glass, and copper. One of the most important results of their work is that based on the distribution of the velocity field, the concentration profile measured at the central part of the cell is correct and is not affected by the convections with velocities between of 10 and $35 \mu \mathrm{m}$.

Later on, Mialdun and Shevtsova [59] studied the thermal design of the cell and optimized it to establish a more linear temperature distribution inside the cell along the vertical line. In result, the convections decreased successfully. It was pointed out that the convection in both the liquid and the surrounding gas strongly affects the quality of the optical measurements.

As discussed, since the discovery of thermodiffusion by Soret and Ludwig, many scientists have tried to measure Soret coefficients of various liquid and gas mixtures using different techniques and operating conditions. In 1999, five different research groups started to establish a benchmark named Fontainebleau providing the Soret coefficients of three binary mixtures composed of dodecane $\left(\mathrm{C}_{12} \mathrm{H}_{26}\right)$, isobutylbenzene (IBB), and 1,2,3,4-tetrahydronaphthalene (THN). The chosen liquids represent alkenes, one-ring, and two-ring components (Figure 2.6) [60]. The techniques used by these five groups were parallelepiped TGC technique along with the openended capillary technique [61], the TDFRS or holographic grating technique [62, 63], an annular TGC, and a packed TGC [64]. Recently, Mialdun and Shevtsova [65] added data from the ground based ODI technique, and Königer et al. [66] added data for the beam deflection technique to the benchmark data. Table 2.1 presents the Soret coefficients from the benchmark and ground based ODI experiments [65]. The good agreement between the ODI results and benchmark values shows the high reliability of the interferometry techniques which are definitely less costly than space experiments. 
<smiles>c1ccc2c(c1)CCCC2</smiles>

1,2,3,4-Tetrahydronaphthalene<smiles>CCCCCCCCCCCCC</smiles>

Dodecane<smiles>CC(C)Cc1ccccc1</smiles>

Isobutylbenzene

Figure 2.6: Molecular structure of the pure components of the hydrocarbon mixture used in Fontainebleau benchmark

Table 2.1: Soret coefficients for mass fraction of 0.50 and temperature of $25{ }^{\circ} \mathrm{C}$

\begin{tabular}{llll}
\cline { 2 - 4 } & & $\mathrm{S}_{\mathrm{T}} ; 10^{-3} \mathrm{~K}^{-1}$ & \\
\hline Description & ${\mathrm{IBB}-\mathrm{C}_{12} \mathrm{H}_{26}}$ & & \\
\hline THN-C ${ }_{12} \mathrm{H}_{26}$ & THN-IBB \\
\hline Benchmark of Fontainebleau [60] & $3.9 \pm 0.1$ & $9.5 \pm 0.5$ & $3.3 \pm 0.3$ \\
\hline Optical Digital Interferometry method [65] & & & $3.29 \pm 0.11$ \\
\hline
\end{tabular}

Although binary mixtures are well- known in terms of Soret coefficients, there is still a lack of information about Soret coefficients of ternary mixtures, and the results that do exist in different reports are not in a good agreement. Firoozabadi et al. [67] for the first time reported the thermodiffusion coefficients of a non-electrolytic ternary mixture. The thermogravitational column method was used to run the experiments. They also measured the molecular diffusion coefficients for binary and ternary mixtures by means of an open-end capillary-tube method. In their work, the binary mixtures were investigated to utilize the method suggested by Larre et al. [68] to estimate thermal diffusion of the ternary mixtures from the binary results. Van Vaerenbergh et al. [69] studied thermodiffusion in multicomponent hydrocarbon mixtures experimentally and computationally. They studied both binary and multicomponent hydrocarbon mixtures at high pressure. Experiments were done in a nearly convection-free environment in space on board the spacecraft FOTON-M3. It was found that the performance of the thermodiffusion model will be deteriorated by increasing the number of components.

According to the Soret effect, when a multicomponent mixture in a Soret cell is subjected to a temperature gradient, components will travel toward the hot or cold side of the cell based on the Soret coefficients of the system. The species that migrate to the cold side are called 
thermophobic and the ones tend to travel to the hot side are named thermophilic. While components are being separated due to the thermal diffusion, concentration-based diffusion occurs to re-homogenize the mixture. In case of a ternary system, two independent concentrations must be resolved. Therefore, two different independent diagnostics are required. In sampling techniques, e.g., TGC, refractive index and density can play the role of the diagnostics. In the optical methods, by using two different wavelengths that come from two separate laser sources, this requirement can be fulfilled. Developing this idea, a thermal diffusion experiment of a mixture with $N$ components using optical methods requires $(N-l)$ lasers with different wavelengths.

Firoozabadi [70] for the first time proposed using a two-colour optical beam deflection method to do thermodiffusion experiments in ternary fluid mixtures. Later, Königer et al. [71] implemented the method. However, only a partial agreement between their results and literature data was confirmed. The tested mixture composed of THN - IBB- $\mathrm{C}_{12}$ with a 1:1:1 ratio of mass fractions and a benchmark of the Soret information was provided [71].

Ahadi and Saghir [72] analysed the thermal diffusion experiment on a ternary mixture in a low gravity environment. A hydrocarbon mixture of THN-IBB- $\mathrm{C}_{12}$ with the mass fraction of 10/80/10 was used in a Soret cell. The Mach-Zehnder interferometry technique including two lasers with different wavelengths was used to measure the Soret coefficients. The successful measurements in their work proves that ODI can be considered a reliable technique to measure Soret coefficients on ground experiments.

Mialdun and Shevtsova [73] analysed the results of TCG[74] and OBD [71] and showed that the true values of the Soret coefficients are highly dependent on the accurate values of contrast factors. In their study, a way is reported to match the results of the three techniques into a unique value.

Recently, six participating laboratories studied the thermal diffusion in a ternary mixture by implementing different techniques. The studied mixture was THN-IBB- $\mathrm{C}_{12}$ with the mass fraction of $80 / 10 / 10$ at a temperature of $25^{\circ} \mathrm{C}$. The benchmark of the Soret and thermal diffusion coefficients of the mixture was prepared and named DCMIX1[75]. The selectable optical diagnostic instrument (SODI) configuration works in microgravity conditions in space. The configuration is equipped with a Mach-Zehnder interferometer with two lasers of different wavelengths. The captured interferogram patterns were analyzed by four teams independently. The 
benchmark values obtained from the ground based experiments and also using the SODI configuration are summarized in Table 2.2.

The Soret coefficient of the first and third components, $\mathrm{THN}$ and $\mathrm{C}_{12}$, are presented. The reason of choosing and presenting the Soret coefficients of these two components is their higher accuracy due to having the highest differences in density and refractive index. The Soret coefficient of the second component is calculated from:

$$
S_{T, 1}^{\prime}+S_{T, 2}^{\prime}+S_{T, 3}^{\prime}=0
$$

Table 2.2: DCMIX1 benchmark values of Soret coefficients [75]

\begin{tabular}{lcc}
\hline & $S_{T, 1}^{\prime} \times 10^{-3}$ & $S_{T, 3}^{\prime} \times 10^{-3}$ \\
& $\left(\mathrm{~K}^{-1}\right)$ & $\left(\mathrm{K}^{-1}\right)$ \\
\hline Ground conditions & $1.11 \pm 0.15$ & $-0.91 \pm 0.08$ \\
Microgravity conditions & $1.37 \pm 0.13$ & $-0.65 \pm 0.19$ \\
\hline
\end{tabular}

The available benchmarks for ternary mixtures covers only a few mass fractions. In order to contribute to establish benchmark for the remained mass fractions an interferometry experimental setup with Mach-Zehnder arrangement is suggested in the present study. The studied mixtures were placed in a convectionless Soret cell which was a re-design of the cell used previously in the space studies. This ground based experiments is more convenient to conduct compared to expensive experiments run in space. However, due to the presence of gravity force in ground, it is necessary to take precautions to avoid the undesirable convective motions.

\subsection{Conclusions}

The importance of the thermal diffusion in petroleum industry inspired scientists to measure thermal diffusion in hydrocarbon mixtures experimentally. Generally, two categories of Soret experimental methods are existed: convectionless and convective coupling methods. To avoid the convective motions due to the gravity force, some experiments have been performed in space which are very costly.

Experimental studies by different research groups have been performed to establish benchmarks of thermal diffusion information for mixtures composed of THN, IBB, and $\mathrm{C}_{12}$ which are the main representatives of crude oil. However there are still lots of mass fractions with missing 
information. Moreover the experimental results in the literature for ternary mixtures are not in a good agreement.

The In order to run a ground-based convectionless test it is necessary to provide the conditions to avoid the motions. A cell which was designed for space experiments was used in ground-based experiments and the strong convective motions in the mixture masked the thermodiffusion. Thereafter, the cell was redesigned and a cell with new geometry was used to perform the experiments. Thermal design of the cell was studied numerically in terms of velocity and temperature profiles. The effect of the inclination of the cell on the convection and thermal diffusion was ignored in other studies by mistake. In this study, for the first time, this effect was investigated. Moreover, accurate measurement of thermal diffusion in ternary mixtures by utilizing the interferometry technique using two lasers with different wavelengths was introduced. 


\section{MATERIALS AND EXPERIMENTAL METHODS}

\subsection{INTRODUCTION}

Tetrahydronaphtalene, isobutylbenzene, and dodecane are known as the representatives of a hydrocarbon reservoir mixture. Mixtures composed of these components with different mass fraction were tested in the current study. As the prepared mixture and lateral glass walls of the Soret cell transparent, laser beams are able to traverse the test section. In the optical interferometry techniques, the changes in refractive index make an optical path difference between two beams and a series of fringes are imaged on a screen. By image processing the created fringe patterns, the refractive index variation is measured. As the mixture is placed in the Soret cell and heated from above, a profile of refractive index is established due to the induced temperature and concentration gradients.

\subsection{Chapter AIMS}

This chapter presents details about the mixtures and mass fractions used in the binary and ternary mixtures. The interferometry technique used to measure temperature and concentration fields is explained and the optical equipment with their applications will be introduced. The Soret cells with different geometries will be explained and after describing the experimental procedure, the applied image processing method will be presented.

\subsection{MATERIAls USED IN THE CELL}

The Soret cell used in this study was made of 5 different materials. The core of the cell, which is the cubic cavity in the centre, was surrounded by windows made of quartz. The quartz glass was clamped between two nickel-plated copper blocks. Buna-N O-rings were used in between the quartz glass and copper blocks for sealing. The entire cell and copper blocks were insulated by Teflon Polytetrafluoroethylene (PTFE) walls. The PTFE walls were fastened around the cell by 12 screws. An injection hole was designed into each of the copper blocks. After filling the cell, these injection holes were closed and sealed by special screws. 


\subsection{FLUID MIXTURES}

The hydrocarbon mixture of $\mathrm{C}_{12}$ - THN of mass fraction $0.50 / 0.50$ at a temperature of $25^{\circ} \mathrm{C}$ was used for the binary experiments. This mixture has been previously studied widely, and thus there is a benchmark of Soret coefficients prepared for the Soret coefficient for this mixture [60]. Therefore, this system was chosen to validate the setup and also study the effect of different parameters on the separation of the components.

The hydrocarbon mixture of THN-IBB- $\mathrm{C}_{12}$ with five different mass fractions presented in Table 3.1 at a temperature of $25^{\circ} \mathrm{C}$ was used for ternary thermal diffusion experiments. The reason of selecting these specific mass fractions is explained in chapter 5 . The mean temperature used in the benchmarks is $25^{\circ} \mathrm{C}$.

The ternary mixture of THN-IBB-C 12 with mass fraction of $80 / 10 / 10$ at a mean temperature of $25{ }^{\circ} \mathrm{C}$ was tested by other groups to contribute to DCMIX-1 benchmark. This mixture was investigated again in the current study in order to make a contribution to the benchmark and also establish a proper optical digital interferometry configuration for further studies on mixtures with different mass fractions.

The chosen components are the main representatives of a hydrocarbon reservoir mixture. All the components were purchased from Fisher Chemical Canada with a purity of more than $98 \%$.

Table 3.1: Mass fraction of the ternary mixtures used in the present study

\begin{tabular}{cccc}
\cline { 2 - 4 } & \multicolumn{3}{c}{ Mass fraction } \\
\hline mixture number & THN & IBB & $\mathrm{C}_{12}$ \\
\hline 1 & 80 & 10 & 10 \\
2 & 70 & 10 & 20 \\
3 & 73.4 & 10.3 & 16.3 \\
4 & 71.8 & 20.2 & 8 \\
5 & 81.9 & 10 & 8.1 \\
\hline
\end{tabular}

\subsection{MiXTURE PREPARATION}

An analytical balance was used to weigh the components of the mixture. The balance used was a Denver Summit Series Instrument balance (Fisher Scientific catalogue numbers of SI-234) with precision of 4 decimal places $\left(10^{-3} \mathrm{mg}\right)$. The objective of the mixture preparation was to fill the container bottle shown by the mixture components to reach the desired mass fraction. 
The inside and outside of the container must be dry and clean before filling. The empty container was placed into the balance. By pushing the "Tare" button, the balance was zeroed. Then the container was taken out and filled by the first component, i.e., THN, and then it was placed into the balance again to weigh it. After reaching the desired weight, the balance must be zeroed again for the second component. By adding the second component with the same weight, the preparation of the binary mixture components in the bottle was completed. Thereafter, the mixture was placed on a stirring device (Fisher Scientific, Isotemp, Basic Stirring Hotplates: Aluminum Top), and in order to homogenize the mixture, a magnetic stir bar is placed into the container to spin in the mixture for few minutes with speed of $200 \mathrm{rpm}$.

The chemicals are toxic and the mixture must be prepared in a fume hood (Mott Labs 7221000 Fume Exhaust Hood).

\subsection{EXPERIMENTAL METHOD}

Optical digital interferometry in a Mach-Zehnder scheme was used to carry out the experiments. This technique is based on the optical path difference between two beams split from a collimated source. The optical path difference is caused by the variation of the index of refraction in the mixture, which is caused by either a temperature or a concentration variation. By image processing of the fringe pattern obtained from the Mach- Zehnder interferometer, the index of refraction distribution in the cell is found.

In this type of interferometry technique, the laser beam passes through the test section only once. Therefore, the fringes produced are sharp and clear[76]. Interferometry technique of measurement is non-intrusive and it does not need a probe and it does not disturb the mixture during the experiment which is essential in convectionless techniques of thermal diffusion experiments.

The general arrangement of the Mach- Zehnder interferometer is shown in Figure 3.1. The interferometry setup for binary and ternary experiments is described here, respectively. 


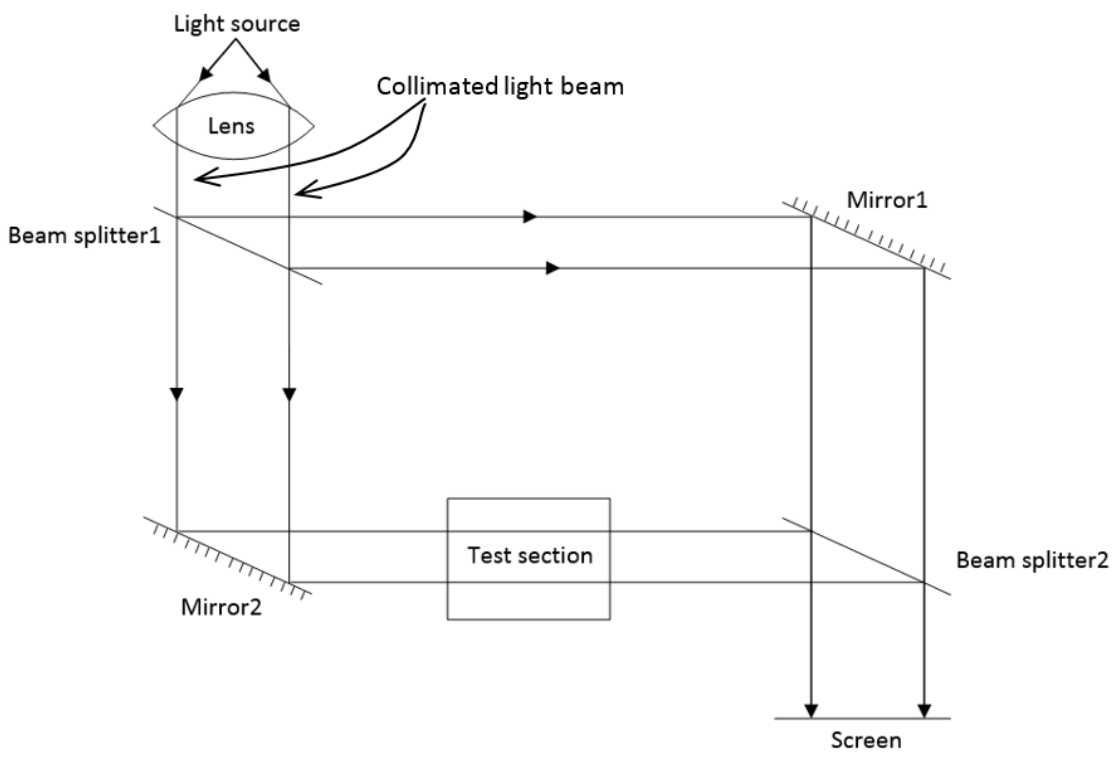

Figure 3.1: Schematic of the Mach-Zehnder Interferometer (MZI)

\subsection{INTERFEROMETRY SETUP FOR THE BINARY TESTS}

The Mach-Zehnder interferometer used for the binary systems included the following equipment:

1- He-Ne Laser: The optical light source used in the interferometer for binary tests was a red cylindrical Helium-Neon laser with wavelength of $632.8 \mathrm{~nm}$ and $15 \mathrm{~mW}$ power (Melles Griot model: 25-LHP-151-249, beam diameter: $0.8 \mathrm{~mm}$ ). The length of the cavity was $396.2 \mathrm{~mm}$. He$\mathrm{Ne}$ lasers with wavelength of $632.8 \mathrm{~nm}$ are used commonly in optical research. The other reason for choosing this wavelength is the popularity of using this device in previous studies in the field of Soret experiments. In the Mach-Zehnder interferometer used in the SODI experiments, the same laser light source was used.

2- Spatial filter: A spatial filter, including a micro-lens and a pinhole, was used to expand the laser beam and to increase the quality of the wavefront. The micro-lens expands the beam. However, the propagated beam from the laser source does not have a perfectly spherical wavefront. The uneven wavefront can cause undesirable interference. To avoid that, a pinhole was used at the distance of one focal length from the micro-lens. Figure 3.2 shows the effect of using the pinhole on the quality of the wavefront. 
The spatial filter used in this study was a three-axis spatial filter (Newport corporation, Model: M-900) shown in Figure 3.3, and the pinhole diameter was 25 micron (Newport corporation, Model: 900PH-25).

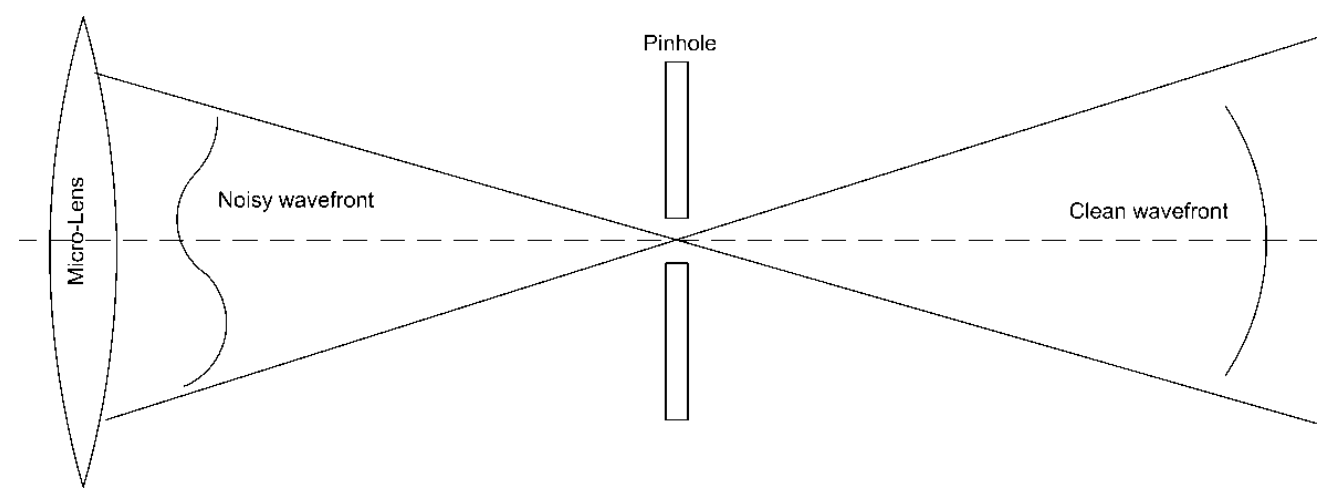

Figure 3.2: Effect of spatial filter on the quality of the wavefront

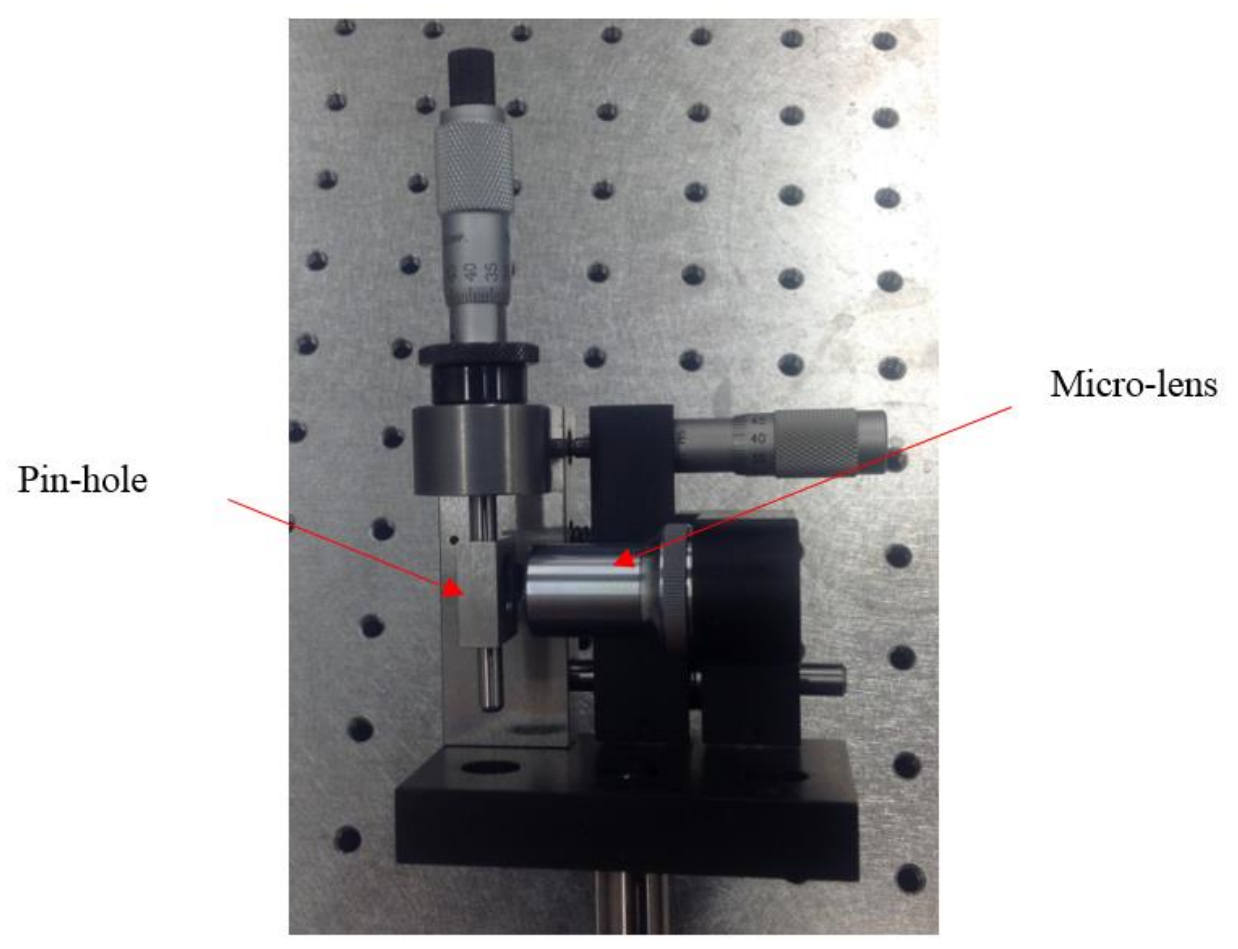

Figure 3.3: Spatial filter

3- Lens: A plano-convex spherical lens was used to collimate the beam. It was located at the distance of its focal length from the light source (Figure 3.4), which was the pin-hole. A shear- 
plate collimation tester was used to test if the beam was collimated properly. The shear-plate was placed after the lens, and as the visible beam entered the plate, fringes appeared on the screen on top of the shear-plate. The fringes parallel to the straight line on the screen indicate proper collimation of the beam. Figure 3.5 shows the collimated, converging or diverging beams. The second plano-convex lens is used to focus the beam on the camera. Among different types of lenses, the plano-convex lens provides the lowest amount of spherical aberration. As shown in Figure 3.6, to reduce the aberration effect, the curved side of the lens must be placed towards the collimated beam.

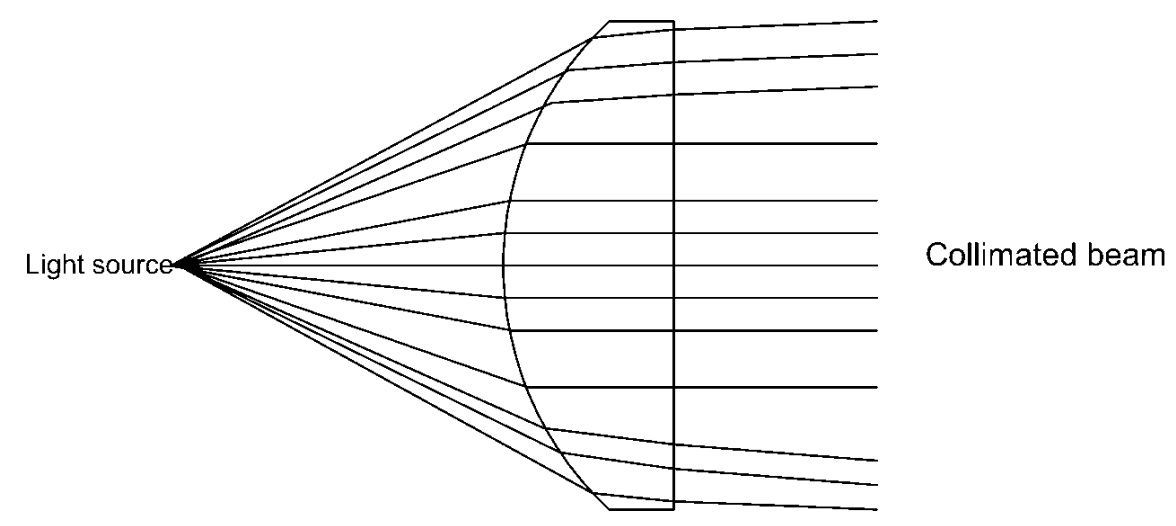

Figure 3.4: Collimating the beam using a Plano-Convex lens

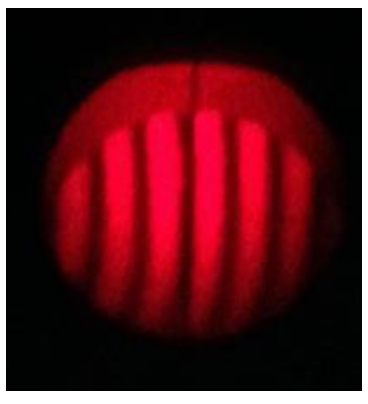

(a) collimated beam
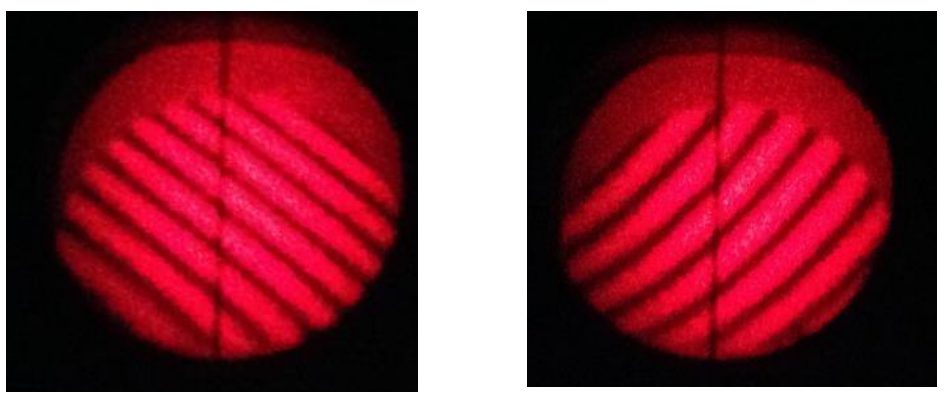

b non-collimated beam

Figure 3.5: Testing the collimation of the beam using a shear-plate collimation tester, showing (a) Collimated beam (b) non-collimated beam 


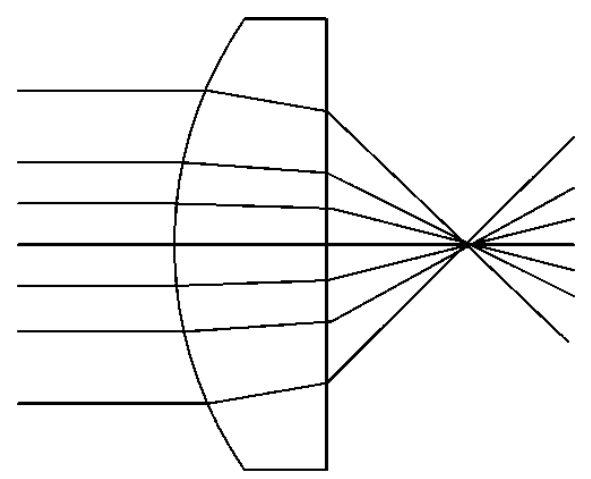

(a) with less aberration

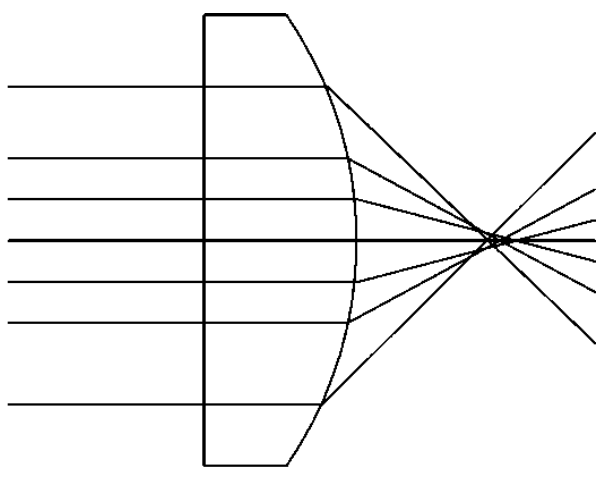

(b) with more aberration

Figure 3.6: Reducing the aberration by choosing the correct side of the lens towards the collimated beam

4- Beam splitter: beam splitters are used either to split the beam into two beams or to recombine beams. The percentage of the reflection varies in different beam splitters and for different wavelengths. There are generally two types of beam splitters: Cube beam splitters and plate beam splitters (Figure 3.7). The cube type consists of two glass prisms with one of them coated. The two prisms are cemented together. Plate beam splitters are flat glass plates with the front surface of the splitter reflecting a portion of the incident beam while the rear surface has an antireflection coating to avoid undesirable reflection from the second surface.

The beam splitter used in this study (BS1-633-50-2025-45P, Melles Griot) was a plate beam splitter, made of fused silica with diameter and thickness of $50.8 \mathrm{~mm}$ and $6.35 \mathrm{~mm}$, respectively. It is a non-polarizing splitter that does not change the polarization state of the incident beam.

Two beam splitters were used in the Mach-Zehnder interferometry setup. The first beam splitter, BS1, split the collimated beam into two separated beams, while the second beam splitter recombines two beams. Thereafter, the recombined beams travel towards the lens and CCD camera. 


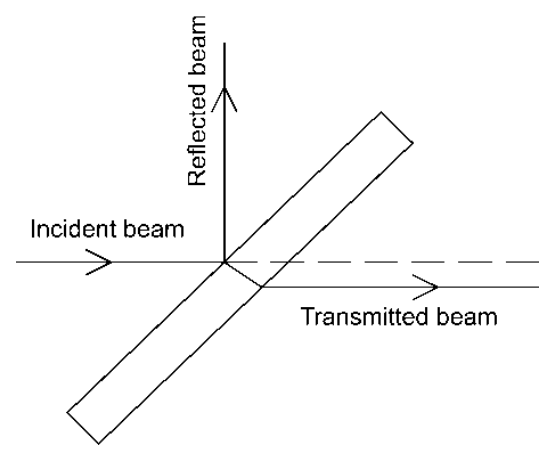

(a) plate beam splitter

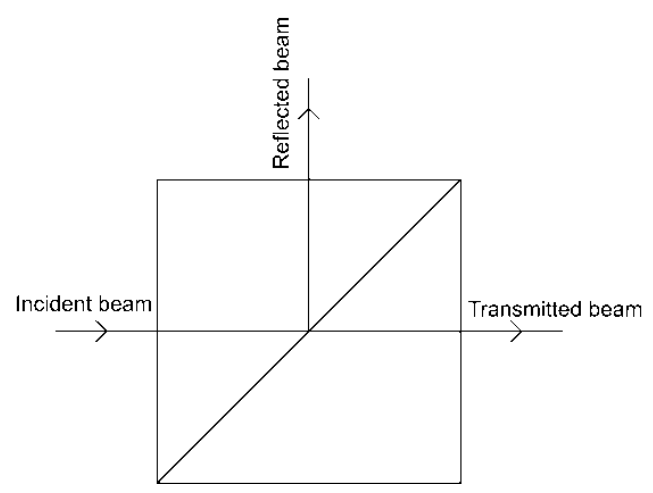

(b) cube beam splitter

Figure 3.7: Beam splitter (a) plate beam splitter (b) cube beam splitter

5- Mirror: A metal-coated mirror (Enhanced Aluminum - EAV-PM-2037-C) with diameter and thickness of $50.8 \mathrm{~mm}$ and $6.35 \mathrm{~mm}$, respectively, was used to reflect the beam. The mirror is made of N-BK7 glass and coated with enhanced aluminum. The average reflectance of these aluminum mirrors is more than 93\%. Two mirrors were used in the MZI interferometer setup. One reflected the reference beam towards the second beam splitter and the other mirror reflected the object beam towards the Soret cell. Mirror mounts (200-20M-3SC-LK) with 3 adjustable screws were used to mount the mirrors on the bench and adjust the fine angles.

6- Detector: In order to image the fringes on the screen, a CCD camera (JAI progressive scan, model: CV-M4-CL+ monochrome digital megapixel camera) was installed. This is a 2/3" megapixel progressive scan camera with 1392 x 1040 effective pixels and an image scale of approximately $6.45 \mu \mathrm{m}$ square pixels at 24 frame per second. A plano-convex lens was used to focus the recombined beams for the lens of the camera. The flat side of the lens faced the camera. The field of view shown on the screen in $15 \mathrm{~mm}$, so the resolution is 90 pixel $/ \mathrm{mm}$.

The beams were emitted from the He-Ne laser at a wavelength of $632.8 \mathrm{~nm}$, and the microlens expanded the beam to pass through the pin hole located at the focal distance of the micro-lens. Thereafter, the first plano-convex lens collimated the beam. The first beam splitter divided the collimated beam into two separated beams and each of them passed one of the arms of the MachZehnder. The beam in the reference arm passed through the undisturbed environment and then was reflected by the first mirror towards the second beam splitter, while the object beam was reflected by the second mirror towards the Soret cell and recombined with the reference beam at the second beam splitter. Finally, the recombined beam was focused on the CCD camera after passing through 
the second plano-convex lens. The whole optical system was mounted on an optical table (Newport corporation, dimension 1.2 × 2.4 × $0.203 \mathrm{~m}$, model: M-RS2000-48-8). The components were installed on mounting posts mounted to the optical table. The posts of the CCD camera and the lens next to it were installed on rail carriers (Newport cooperation, model: PRC-1), which can slide on a precision optical rail (Newport cooperation, model: PRL-6). So, the camera and the lens can be moved in order to find the best location where sharp and clear images are shown on the screen.

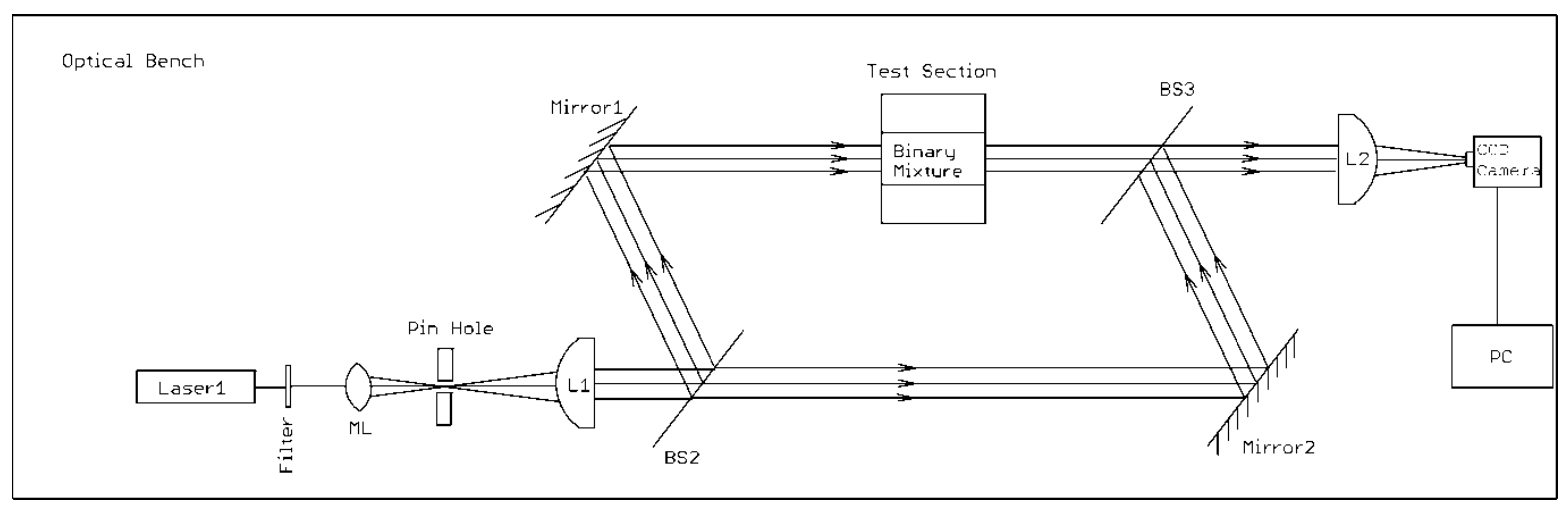

Figure 3.8: Interferometry set-up for binary tests

\subsection{INTERFEROMETRY SETUP FOR THE TERNARY EXPERIMENTS}

In the Mach-Zehnder arrangement for ternary systems, two lasers with different wavelengths were used at the same time (Figure 3.9). In order to use the same Mach-Zehnder interferometer for both lasers, they are placed on the optical bench perpendicular to each other. One laser emits the laser beam in a straight line towards the MZI setup, while the laser beam of the other laser source is reflected towards the setup by a beamsplitter. Here, a beam splitter is used instead of the mirror to avoid blocking the first laser. Laser sources with wavelengths of 632.8 and $940 \mathrm{~nm}$ were used in this thesis. Sechenyh et al. [77] found that the choice of these wavelengths is not optimal. However, these wavelengths were chosen for this study in order to have a similar setup as that used during the microgravity experiments from SODI. 


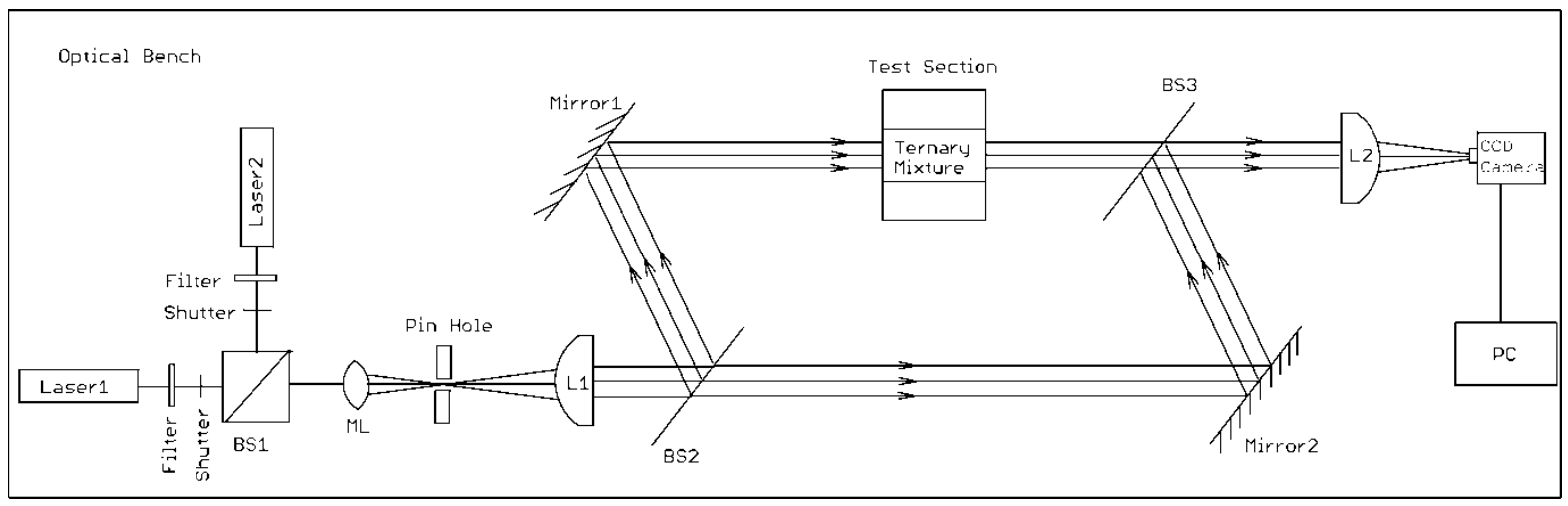

Figure 3.9: Interferometry set-up for ternary test

\subsection{SORET CELL}

The Soret cell used in this study was designed based on the classical Soret cell with heating from above. Previously, Rahman and Saghir[78] used a cell with height and width of 5 and $10 \mathrm{~mm}$, respectively, for binary experiments. As shown in Figure 3.10, the quartz glass window was clamped between two copper blocks. Plastic screws were used to tighten the cell. The mixture was injected into the cell through the injection holes on the copper blocks. Although the heating process was from above, residual convection was unavoidable. However, it is possible to reduce convection by redesigning the cell. The schematic of the new design is shown in Figure 3.11. The dimension of the cavity was $15 \mathrm{~mm} \times 15 \mathrm{~mm} \times 2.5 \mathrm{~mm}$ (width, length, height). The window was made of quartz glass ordered from Hellma Company. It was clamped between the copper blocks. Buna-N O-rings were used in between the glass and the copper blocks for sealing purposes. The injection holes on the copper blocks are shifted to the corners, which will be explained in more detail in chapter 4 . An inclined hole was made in one side of each copper block to place a thermistor in it. Calibrated PT100 thermistors were used to measure the temperature, and two electronic proportional-integral-derivative (PID) controllers regulated the temperature of each block independently with precision of less than $\pm 0.01^{\circ} \mathrm{C}$. PTFE walls were used to tighten and assemble the system and insulate it at the same time. Windows were designed on the back and front walls to let the laser beam pass through the cell. The PTFE walls were connected to each other by screws. 


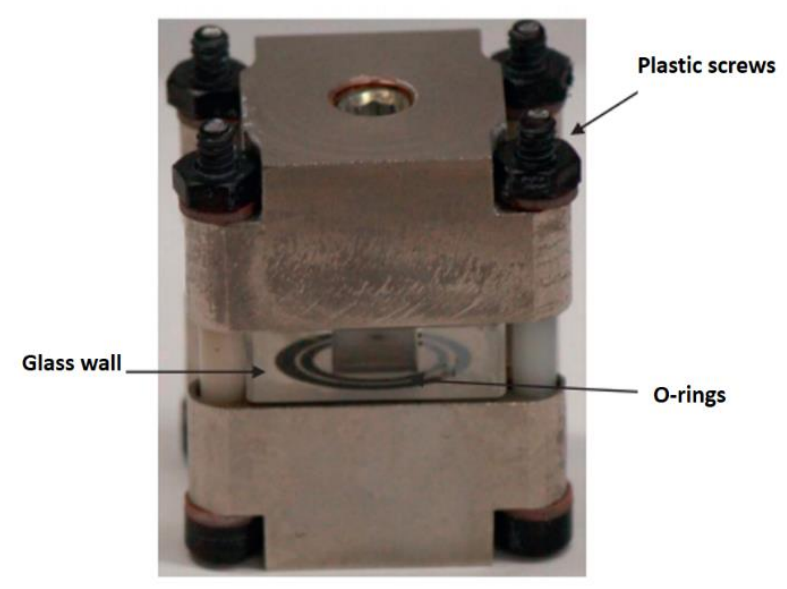

Figure 3.10: Soret cell used in the previous studies [78]
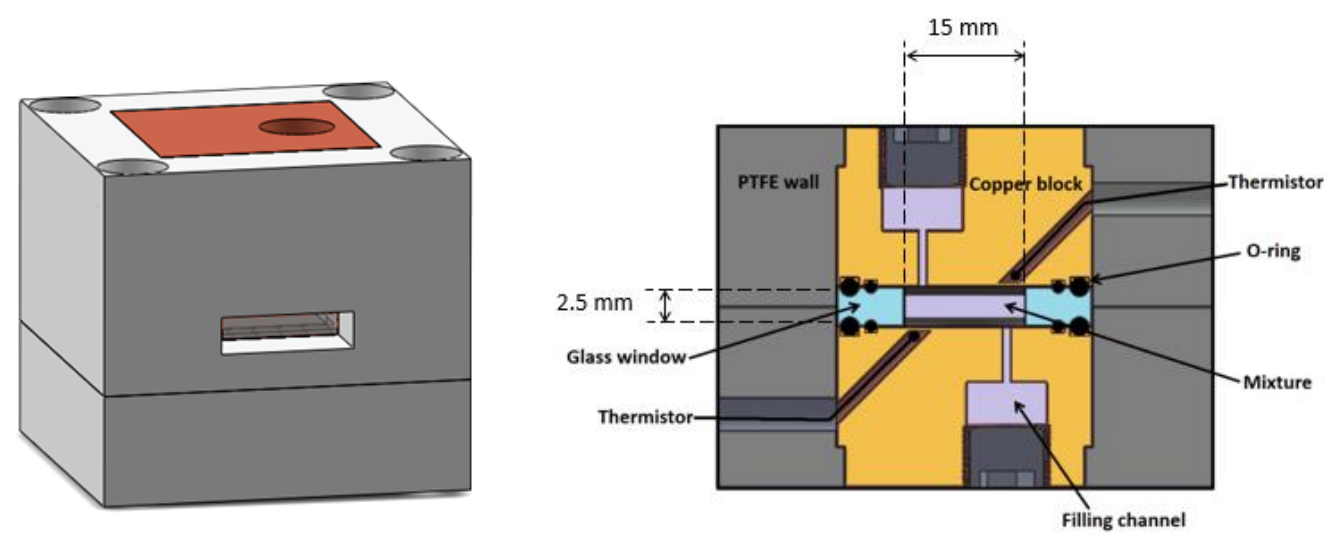

Figure 3.11: Schematic of the new model

To assemble the cell, the first step was to place the Buna-N O-rings into the designated grooves on the copper blocks. Next, the quartz window was placed and clamped between the blocks. Finally, the PTFE walls tightened the cell.

\subsubsection{Filling the cell}

A syringe with a luer- lock taper connection was used to fill the cell with the liquid mixture. By using this type of syringe, a leak-free connection between the syringe head and the filling 
channel on the copper block can be established. While the cell is held vertically, the filling procedure started from the bottom side. The filling must be done continuously until no bubbles remain. Next, the hole on the upper block was fastened with a cap screw. For sealing purposes, Teflon tape was used to wrap the screw. Then, the cell was flipped and the syringe was disconnected. Then the hole was closed with another Teflon wrapped screw. However, before closing the hole there must be no bubbles left in the cell. To remove the bubbles, a needle syringe inserted from the top was used to degas the mixture.

\subsubsection{Installing the cell on the bench}

As mentioned earlier, the Soret effect in this work was studied in the Soret cell by heating from above. The top and bottom copper blocks play the role of the hot and cold sides of the cell, respectively. The constant temperatures of these sides were provided by Peltier elements. One side of each Peltier element was attached to the relevant block and the other side was in contact with a circulating water bath (Fisher scientific, Model: Isotemp 3016D) with a constant temperature. To enhance the heat transfer, thin layers of thermally conductive epoxy was used on the surfaces in contact with the Peltier elements. Plastic holders manufactured manually in the workshop were used to enclose the cell between the water circulators and the Peltier elements.

The Soret cell was installed on three different moveable stages shown in Figure 3.12 which are used to align the cell properly. A jack stage (Newport Cooperation, model: M-EL80) was used to adjust the system vertically to make sure that the laser beam passes through the cell. Figure 3.13 shows the different angles that the cell can have with the coordinate system. The laser beam must traverse the cell perpendicular it. Thus, the angles of $\gamma$ and $\phi$ must be equal to zero. A rotary stage with the diameter of $70 \mathrm{~mm}$ purchased from Newport and a tilt stage (Edmund optics, Stock No. \#66-517) were used to align the cell perpendicular to the laser beam. The angle of $\theta$ was used to study the effect of the inclination on the thermal diffusion. This angle was adjusted by the tilt stage. 


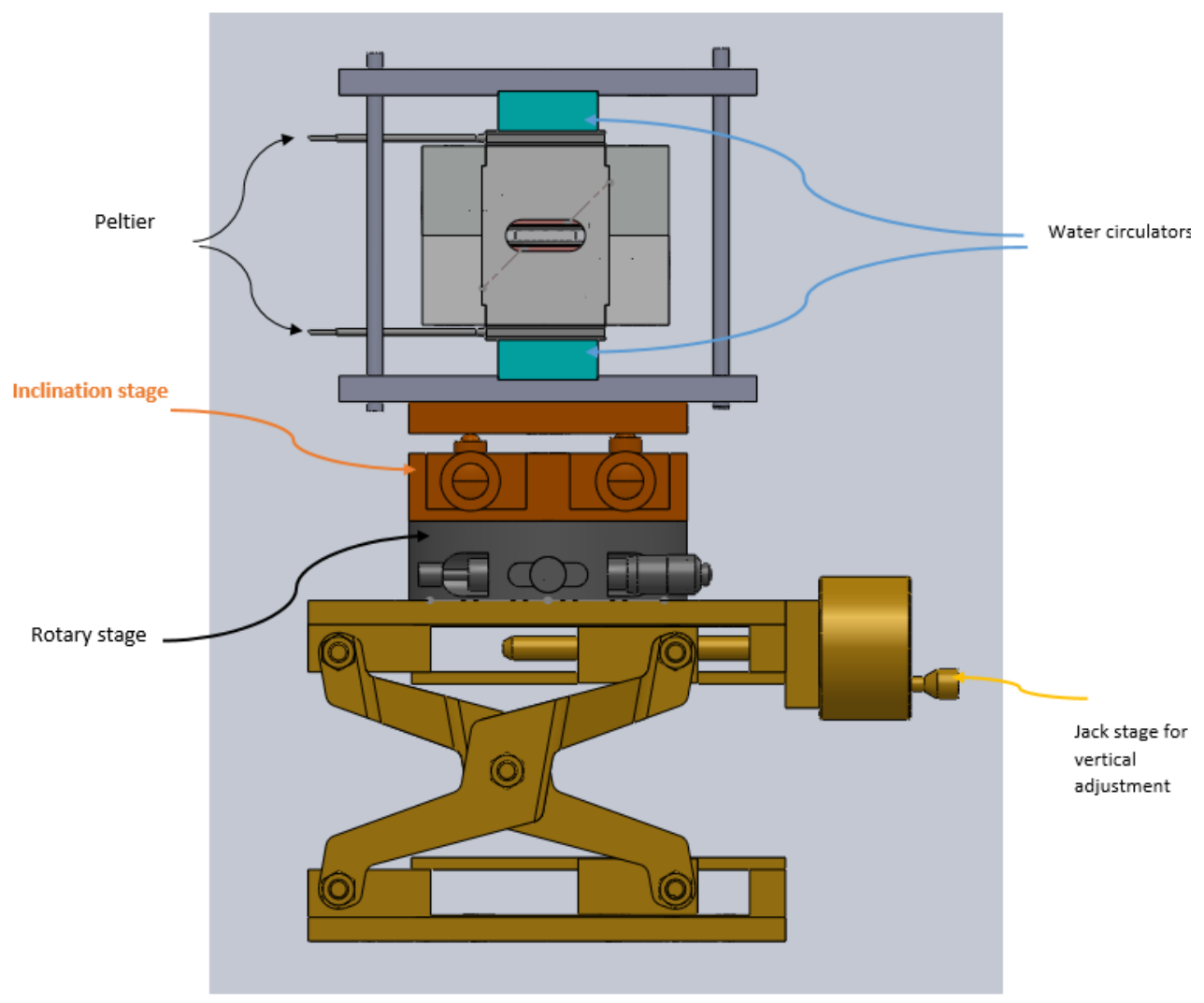

Figure 3.12: Soret cell installed on the inclination stage

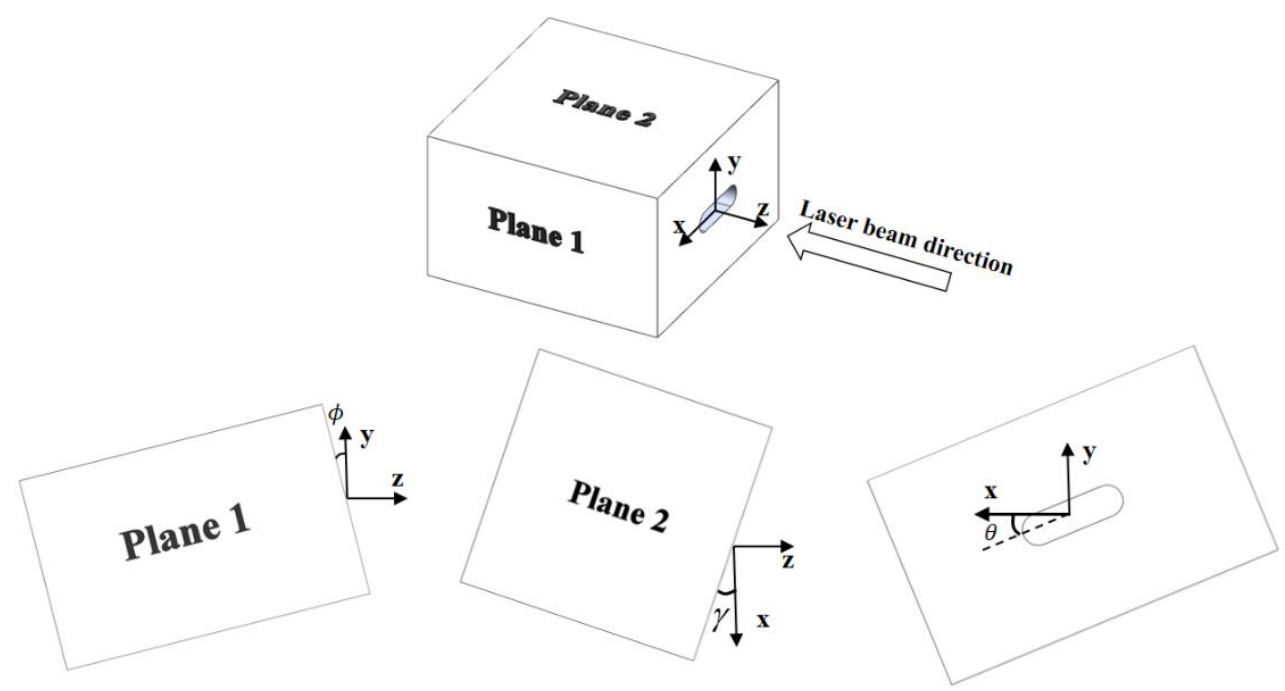

Figure 3.13: Schematic of the cell with alignment angles 


\subsection{EXPERIMENT PROCEDURE}

Both binary and ternary tests have the same procedure, but for the latter, two laser sources were used instead of one. However, only one laser passed through the cell at a time. After filling the cell with the homogenized mixture and installing it on the optical bench, a constant temperature of $25{ }^{\circ} \mathrm{C}$ was applied to the both lower and upper sides of the cell. After a few minutes, a temperature gradient of $1^{\circ} \mathrm{C} / \mathrm{mm}$ was applied between the sides. For instance, in a cell with height of $5 \mathrm{~mm}$, the upper and lower sides would reach the temperatures of $27.5^{\circ} \mathrm{C}$ and $22.5^{\circ} \mathrm{C}$, respectively. The separation of the components commences on application of the temperature gradient, and it continues until the maximum separation happens. The time to reach the maximum separation, steady state, is named the diffusion characteristic time or relaxation time, $\tau_{D}$ :

$$
\tau_{D}=H^{2} / D
$$

For binary mixtures in the Fontainebleau benchmark for a cell with height of $5 \mathrm{~mm}, \tau_{D}$ is approximately 12 hours.

\subsection{IMAGE PROCESSING}

In this study, changes in the temperature and concentration fields cause the variation of the index of refraction, $\Delta n$, that can be measured by image processing of the fringe patterns obtained from the digital interferometry with wavelength of $\lambda$ and initial values of $\mathrm{T}_{0}$ and $\mathrm{C}_{0}$ :

$$
\Delta n(x, z)=\left(\frac{\partial n}{\partial T}\right)_{T_{0}, C_{0}, \lambda} \Delta T(x, z)+\left(\frac{\partial n}{\partial C}\right)_{T_{0}, C_{0}, \lambda} \Delta C(x, z)
$$

$\Delta n$ is obtained from interferometry as follows:

$$
\Delta n=n(x, z)-n\left(x_{0}, z_{0}\right)=\frac{\lambda}{2 \pi L} \Delta \varphi
$$


where $\Delta \varphi$ is the change of the optical phase and can be measured by interferometry and FFT.

\subsubsection{Fast Fourier Transform (FFT)}

Kreis[79] introduced a method using one- or two- dimensional Fourier transforms to determine the phase in interferometry. A typical fringe pattern of the present study is shown in Figure 3.14. The intensity of the pixels ranges between 0 and 255 and have 8 bits. The intensity can be written as[80]:

$$
g(x, y)=a(x, y)+b(x, y) \cos \left[2 \pi f_{0} x+\phi(x, y)\right]
$$

where, $\phi(x, y)$ is the phase distribution containing information about the concentration and temperature fields, $a(x, y)$ and $b(x, y)$ are undesired distributions of the irradiant, $a(x, y)$ represents the background variations, $b(x, y)$ represents the local contrast of the fringe- carrier frequency, and $f_{0}$ is the spatial-carrier frequency. Equation (3.4) can be rewritten as:

$$
\begin{gathered}
g(x, y)=a(x, y)+c(x, y) \exp \left(2 \pi i f_{0} x\right)+c^{*}(x, y) \exp \left(-2 \pi i f_{0} x\right) \\
c(x, y)=\frac{b(x, y)}{2} \exp [i \phi(x, y)]
\end{gathered}
$$

In Equation (3.6) * denotes a complex conjugate. By Fourier transforming the Equation (3.5) with respect to $\mathrm{x}$ by means of a fast Fourier transform the following equation is obtained:

$$
I(f, y)=A(f, y)+C\left(f-f_{0}, y\right)+C^{*}\left(f-f_{0}, y\right)
$$

where $f$ represents the spatial frequency in the $\mathrm{x}$ direction and the capital letters indicate the Fourier spectra. The background variations are shown by $A(f, y)$. So, background variations can be easily filtered out by removing $A(f, y)$ from the spectra. $C$ and $C^{*}$ are symmetrical parts. Therefore, one of them can be picked and the other filtered. In the next step, $C\left(f-f_{0}, y\right)$ is shifted 
to the origin by $f_{0}$ on the frequency axis. Finally, by applying the inverse transform of $C(f, y)$ with respect to $f$ the phase is found:

$$
\phi(x, y)=\tan ^{-1}\{\operatorname{Im}[c(x, y)] / \operatorname{Re}[c(x, y)]\}
$$

$R e$ and $I m$ stand for the real and imaginary parts of the phase, respectively.

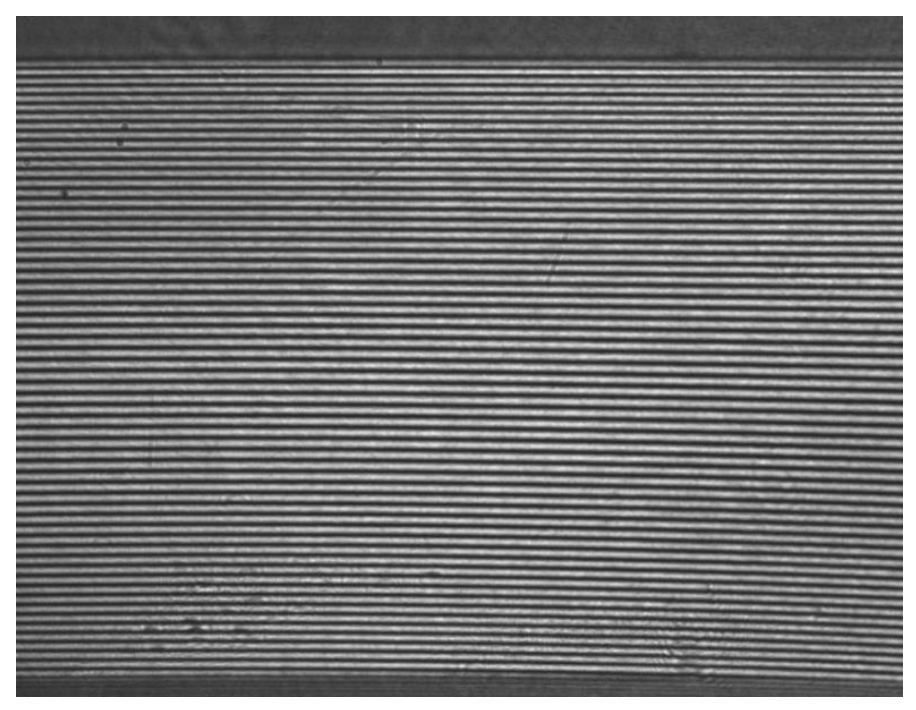

Figure 3.14: A typical fringe pattern captured from the cell in the current study

\subsubsection{Phase unwrapping}

The phase distribution obtained from Equation (3.8) is discontinuous. As shown in Figure 3.15 , a typical phase pattern consists of phase bands, with each phase band ranges between $-\pi$ and $+\pi$. In a continuous phase pattern, the phase difference between two adjacent pixels is less than $\pi$. 


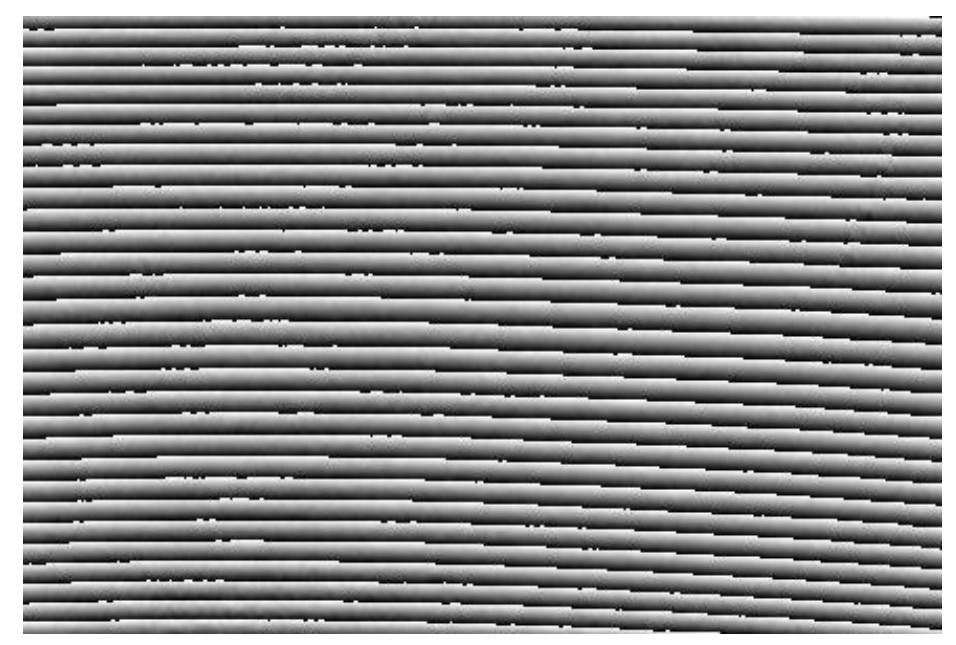

Figure 3.15: Discontinuous phase pattern

The following equation provides the continuous phase of a monotonically increasing phase distribution:

$$
\begin{aligned}
& n\left(x_{1}\right)=0 \text {, } \\
& n\left(x_{i}\right)= \begin{cases}n\left(x_{i-1}\right) & \left|\phi\left(x_{i}\right)-\phi\left(x_{i-1}\right)\right|<\pi \\
n\left(x_{i-1}\right)+1 & \left|\phi\left(x_{i}\right)-\phi\left(x_{i-1}\right)\right| \geq \pi\end{cases} \\
& i=2,3, \ldots \\
& \phi_{\text {contin }}\left(x_{i}\right)=\phi\left(x_{i}\right)+2 \pi n\left(x_{i}\right) \quad i=1,2,3, \ldots
\end{aligned}
$$

For the non-monotonically rising phase distribution the following equation is used:

$$
\begin{aligned}
& n\left(x_{1}\right)=0, \\
& n\left(x_{i}\right)= \begin{cases}n\left(x_{i-1}\right) & \left|\phi\left(x_{i}\right)-\phi\left(x_{i-1}\right)\right|<\pi \\
n\left(x_{i-1}\right)+1 & \phi\left(x_{i}\right)-\phi\left(x_{i-1}\right) \leq-\pi \\
n\left(x_{i-1}\right)-1 & \phi\left(x_{i}\right)-\phi\left(x_{i-1}\right) \geq \pi\end{cases} \\
& \phi_{\text {contin }}\left(x_{i}\right)=\phi\left(x_{i}\right)+2 \pi n\left(x_{i}\right) \quad i=2,3, \ldots
\end{aligned}
$$


For two dimensional phase distribution, as in this study, unwrapping process is done in both $\mathrm{x}$ and $\mathrm{y}$ direction:

$$
\begin{aligned}
& n\left(x_{1}, y_{1}\right)=0, \\
& n\left(x_{1}, y_{i}\right)=\left\{\begin{array}{lc}
n\left(x_{1}, y_{i-1}\right) & \left|\phi\left(x_{1}, y_{i}\right)-\phi\left(x_{1}, y_{i-1}\right)\right|<\pi \\
n\left(x_{1}, y_{i-1}\right)+1 & \phi\left(x_{1}, y_{i}\right)-\phi\left(x_{1}, y_{i-1}\right) \leq-\pi \\
n\left(x_{1}, y_{i-1}\right)-1 & \phi\left(x_{1}, y_{i}\right)-\phi\left(x_{1}, y_{i-1}\right) \geq \pi
\end{array}\right. \\
& n\left(x_{j}, y_{i}\right)=\left\{\begin{array}{lc}
n\left(x_{j-1}, y_{i}\right) & i=2, \ldots \\
n\left(x_{j-1}, y_{i}\right)+1 & \left|\phi\left(x_{j}, y_{i}\right)-\phi\left(x_{j-1}, y_{i}\right)\right|<\pi \\
n\left(x_{j}, y_{i}\right)-1 & \phi\left(x_{j}, y_{i}\right)-\phi\left(x_{j-1}, y_{i}\right) \leq-\pi
\end{array}\right. \\
& \phi_{\text {contin }}\left(x_{i}, y_{i}\right)=\phi\left(x_{j-1}, y_{i}\right) \geq \pi
\end{aligned}
$$

The phase difference between an image and the reference image is used to find the desired variations:

$$
\Delta \phi\left(x, y, t_{i}\right)=\phi\left(x, y, t_{i}\right)-\phi\left(x, y, t_{0}\right)
$$

$\phi\left(x, y, t_{i}\right)$ represents the phase distribution of an arbitrary fringe pattern captured during the experiment. $\phi\left(x, y, t_{0}\right)$ is the phase pattern of the reference image. The reference image is taken when the temperature gradient is not applied on the cell yet.

The obtained phase distribution from the Equation (3.12) is used to find the changes in the index of refraction caused by the temperature and concentration gradients along the optical path of the laser beam in the Soret cell:

$$
\Delta \phi\left(x, y, t_{i}\right)=\frac{2 \pi}{\lambda} \int_{l_{1}}^{l_{2}}\left\{n\left(x, y, t_{i}\right)-n_{0}\left(x, y, t_{0}\right)\right\} d l
$$


Where $l$ indicates the optical length of the Soret cell. It is assumed that the variations of the index of refraction along the optical path is negligible which means $\frac{\partial n}{\partial l}$ is equal to zero. So, Equation (3.13) is simplified to:

$$
\Delta n\left(x, y, t_{i}\right)=\frac{2 \pi}{\lambda} \Delta n\left(x, y, t_{i}\right) l
$$

In binary systems the changes of refractive index in contributed by the changes of the temperature and concentration:

$$
\Delta n(x, z)=\left(\frac{\partial n}{\partial T}\right) \Delta T(x, z)+\left(\frac{\partial n}{\partial C}\right) \Delta C(x, z)
$$

In a ternary system, concentration of two components, $\mathrm{C}_{1}$ and $\mathrm{C}_{2}$, are independent and then $\mathrm{C}_{1}+\mathrm{C}_{2}+\mathrm{C}_{3}=1$ gives the value of $\mathrm{C}_{3}$. To find the independent concentrations from variation of refractive index, the following equation must be solved:

$$
\Delta n(x, z)=\left(\frac{\partial n}{\partial T}\right) \Delta T(x, z)+\left(\frac{\partial n}{\partial C_{1}}\right) \Delta C_{1}(x, z)+\left(\frac{\partial n}{\partial C_{2}}\right) \Delta C_{2}(x, z)
$$

Thermal time is approximately 3-9 minutes [8] and Equation (3.16) in thermal time is simplified to:

$$
\Delta n(x, z)=\left(\frac{\partial n}{\partial T}\right) \Delta T(x, z)
$$

From the latter equation, temperature profile is obtained. After thermal time, the refractive index keeps changing due to the changes in concentration field:

$$
\Delta n(x, z)=\left(\frac{\partial n}{\partial C_{1}}\right) \Delta C_{1}(x, z)+\left(\frac{\partial n}{\partial C_{2}}\right) \Delta C_{2}(x, z)
$$


Equation (3.18) includes two unknowns and cannot be solved. Therefore, another laser with a different wavelength must be used to have the second equation:

$$
\Delta n_{2}(x, z)=\left(\frac{\partial n}{\partial C_{1}}\right)_{\lambda_{2}} \Delta C_{1}(x, z)+\left(\frac{\partial n}{\partial C_{2}}\right)_{\lambda_{2}} \Delta C_{2}(x, z)
$$

Now, having contrast factors, $\left(\frac{\partial n}{\partial C_{i}}\right)_{j}$, concentration profiles are obtainable. The unwrapped phase pattern of a fringe pattern is shown in Figure 3.16.

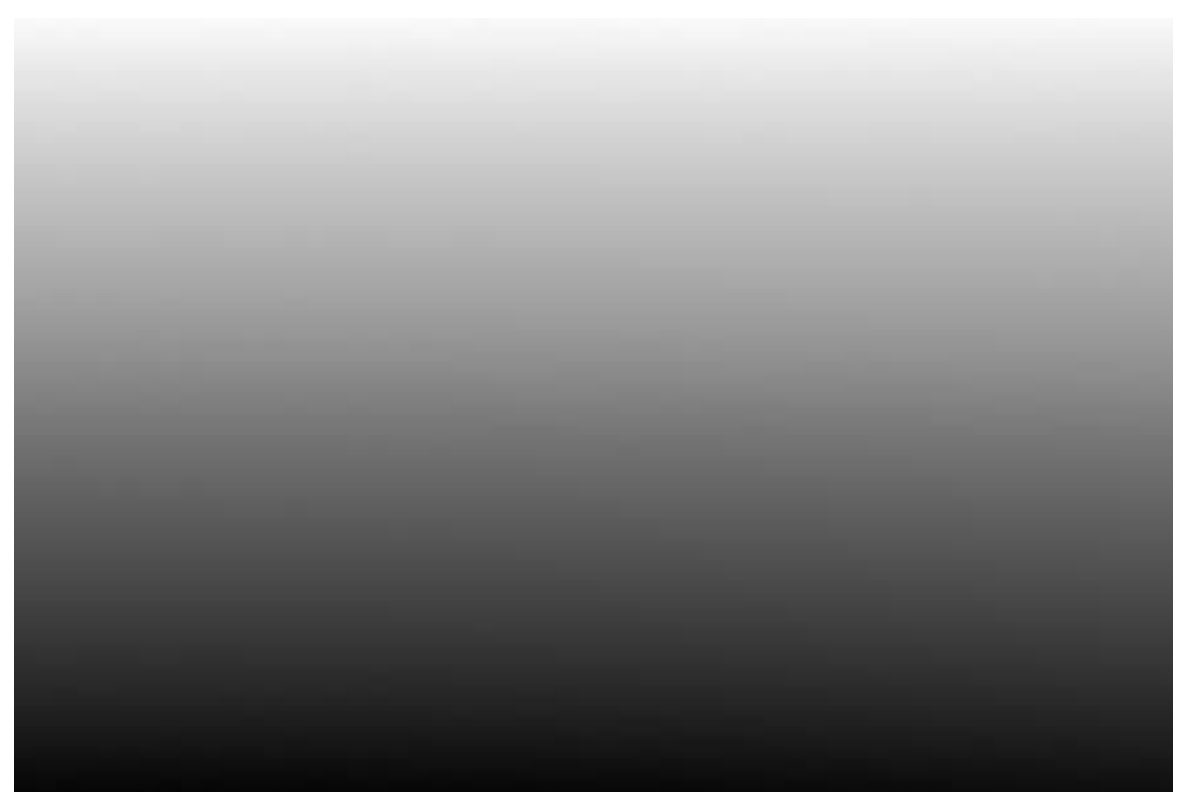

Figure 3.16: Unwrapped phase distribution

\subsection{Conclusions}

A hydrocarbon mixture composed from THN, IBB, and $\mathrm{C}_{12}$ was placed in a Soret cell heated from above. Two different geometries for the cell were used. The applied temperature gradient was $1{ }^{\circ} \mathrm{C} / \mathrm{mm}$. The interferometry technique with Mach-Zehnder arrangement was used as the measurement tool. The laser source used for binary systems had the wavelength of $632.8 \mathrm{~nm}$ while in the ternary systems two laser sources with different wavelengths were used. After 
capturing the fringe patterns formed due to the optical path difference between beams, a series of data analysis were performed to extract the phase information form the fringes. The changes in the refractive index and then concentration and temperature fields were found from the phase changes. 


\section{FACTORS AFFECTING THE EXPERIMENT}

\subsection{INTRODUCTION}

Generally, two kinds of factors which can have deleterious effects on the convectionless measurement of thermal diffusion in this study: interferometry-related factors and thermodiffusion-related factors.

From an interferometry perspective, misalignments of the optical equipment can be sources of error. For instance, improper location of the plano-convex lens next to the camera can increase the beam deflection, which is undesirable.

Besides the factors affecting the optical measurement, there are factors that can affect the thermal diffusion process. Formation of air bubble in the mixture disturbs the segregation of the components. Moreover, inadequate thermal design of the cell increases the convection residuals in the mixture during the thermal diffusion step of the experiment which has a deleterious effect and it prevents the separation.

Thanks to the interferometry technique, the temperature and concentration fields in the mixture can be obtained experimentally. In addition, any bubble in the cell can be identified in the interference images. By utilizing some practical methods that are presented in this chapter, the formation of the bubbles was avoided.

To find the best thermal design of the cell, some numerical studies were performed. Two different geometries were analysed and compared. The effect of the location of the filling channel on the convective motions and temperature profile was examined and the optimized geometry with weaker convections was found. Then, the effect of the inclination of the cell on the thermodiffusion process was studied numerically and experimentally for the optimized cell.

\subsection{CHAPTER AIMS}

In the first part of this chapter, the optical challenges are discussed and it is shown that how beam deflection can reduce the quality of the images. Next, the problem caused by the air bubbles is explained and it is shown that how to solve the problem. The thermal analysis of the Soret cells 
with different geometries is presented and the performance of both designs of the cell is studied numerically and experimentally. Finally the effect of the inclination of the cell on the thermal diffusion is investigated.

\subsection{OPTICAL CHALLENGES}

\subsubsection{Beam deflection problem}

Interferometry can be used to obtain the refractive index profile induced due to temperature and concentration gradients in the mixture. The path that the beam passes through in the mixture is called the optical path. The beam is deflected while traversing the optical path because of variations in the refractive index. There are two types of errors introduced by the deflection of the propagated beam:

1- The interferograms made by the interference of the deflected beams are placed lower than the location that they should be. To explain it visually, a typical fringe pattern of a high deflected beam is shown in Figure 4.1(a). The first figure shows the fringe pattern on the mixture and the side walls before applying the temperature gradient. By applying the temperature gradient, the refractive index profile is introduced in vertical direction in the mixture. As shown in Figure 4.1(b) the fringe pattern on the mixture is located lower than that for the glass wall. The variation of index of refraction of the glass wall is negligible. Therefore, the beam deflection in the walls is negligible and the location of the fringes of the wall is correct.

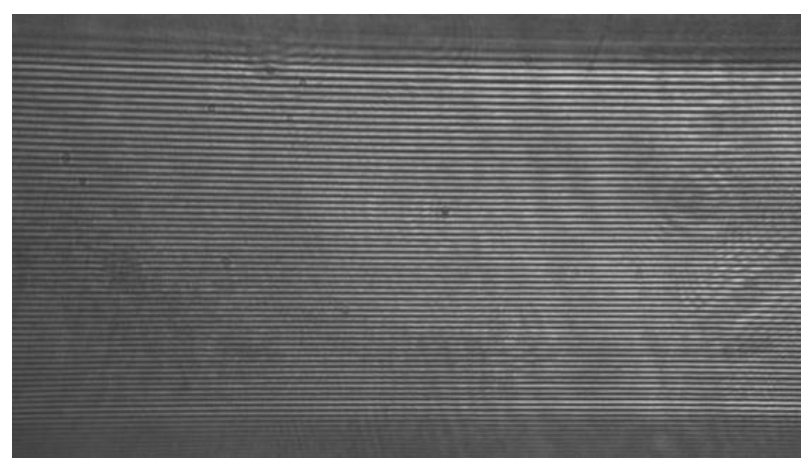

(a) Before applying thermal gradient

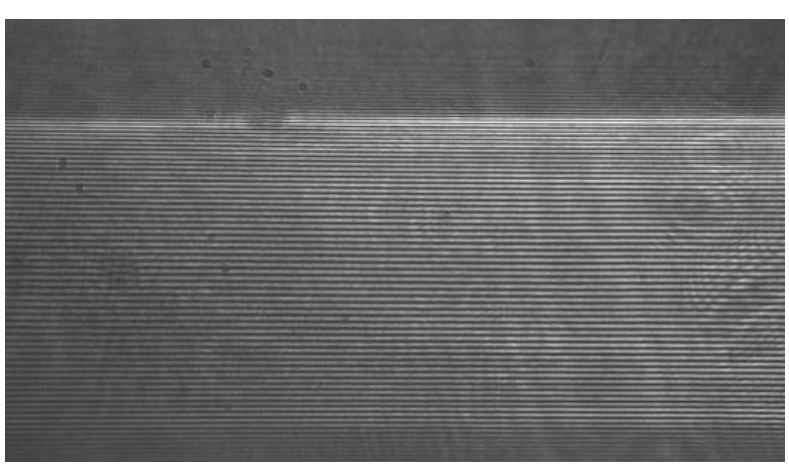

(b) After applying thermal gradient

Figure 4.1: Fringe pattern made by deflected beams 
2- The deflected beam travels a longer distance than the optical length of the cell (Figure 4.2). In this case, the resulting fringes carry incorrect information about the refractive index of the mixture, which leads to the calculation of the incorrect temperature and concentration gradients.

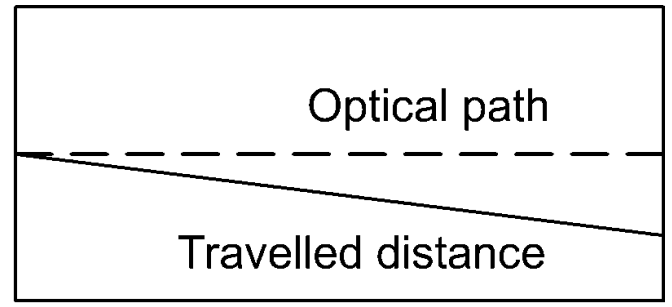

Figure 4.2: Distance travelled and optical path in the cell with beam deflection

As discussed earlier, by applying temperature gradient, a refractive index profile is established in the mixture. The refractive index, $n$, is shown as:

$$
n=\frac{c}{v}
$$

Where $c$ is the speed of light in a vacuum and $v$ is the speed of light in the medium. So, different points of a wavefront in the medium with different refractive indices travel different distances depending on the speed of light at that point. Figure 4.3 shows the deflection of a wavefront travelling in the medium with refractive index profile.

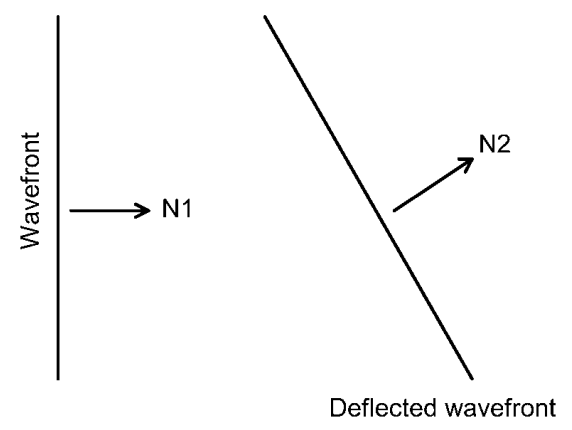

Figure 4.3: Deflected wavefront in the medium with a refractive index profile

Huygen's principle is used to explain the beam deflection. Based on this principle, a wavefront consists of several point sources which produce spherical wavelets. The new location 
of the wavefront after $t$ seconds is the tangent line on the all wavelets (Figure 4.4). As each point has a different velocity because of the variation of the refractive index, the radius of each circle $(r=v \times t)$ increases with time. Therefore, the new wavelet is tilted or in other words the beam is deflected.

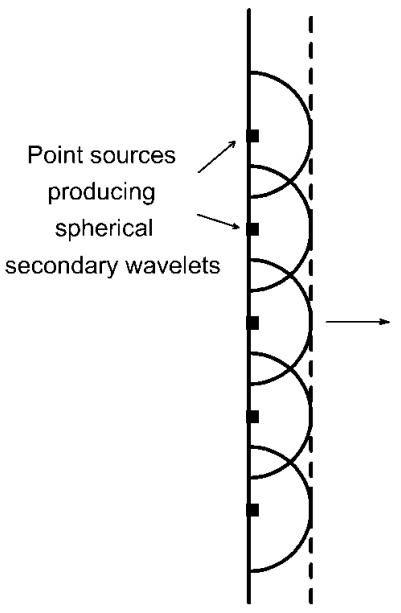

Homogenous media

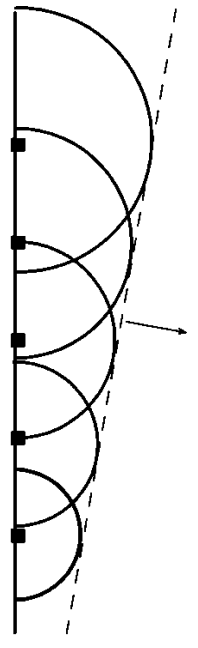

Non-homogenous media

Figure 4.4: Huygen's principle

There are several different interferometry techniques available, e.g., Mach-Zehnder, Rayleigh, Guoy, and Michelson. The beam deflection problem occurs in the all schemes, but in the case of Mach-Zehnder interferometry this problem can be treated easily [65].

By adjusting the location of the focal plane on the inner side of the glass wall nearest to the $\mathrm{CCD}$, the deflection of the beams not close to the bottom boundary can be minimized. This idea was first proposed by Matisson, et al.[81].

In a Soret cell, the lower boundary plays the role of the cold side, and the refractive index of the cold region inside the cell is higher than that of the upper region. The beam deflection occurs towards the cold side. Therefore, a portion of the deflected beam is reflected by the cold plate as shown in Figure 4.5. Consequently, the fringes from the deflection near the boundaries are not as sharp and clear as the fringes created from the deflection around the middle region (Figure 4.6), and therefore the exact concentration difference between boundaries cannot be found. To bypass this problem, the fringes with some few pixels, e.g. 20 pixels near the boundaries are cropped off. 


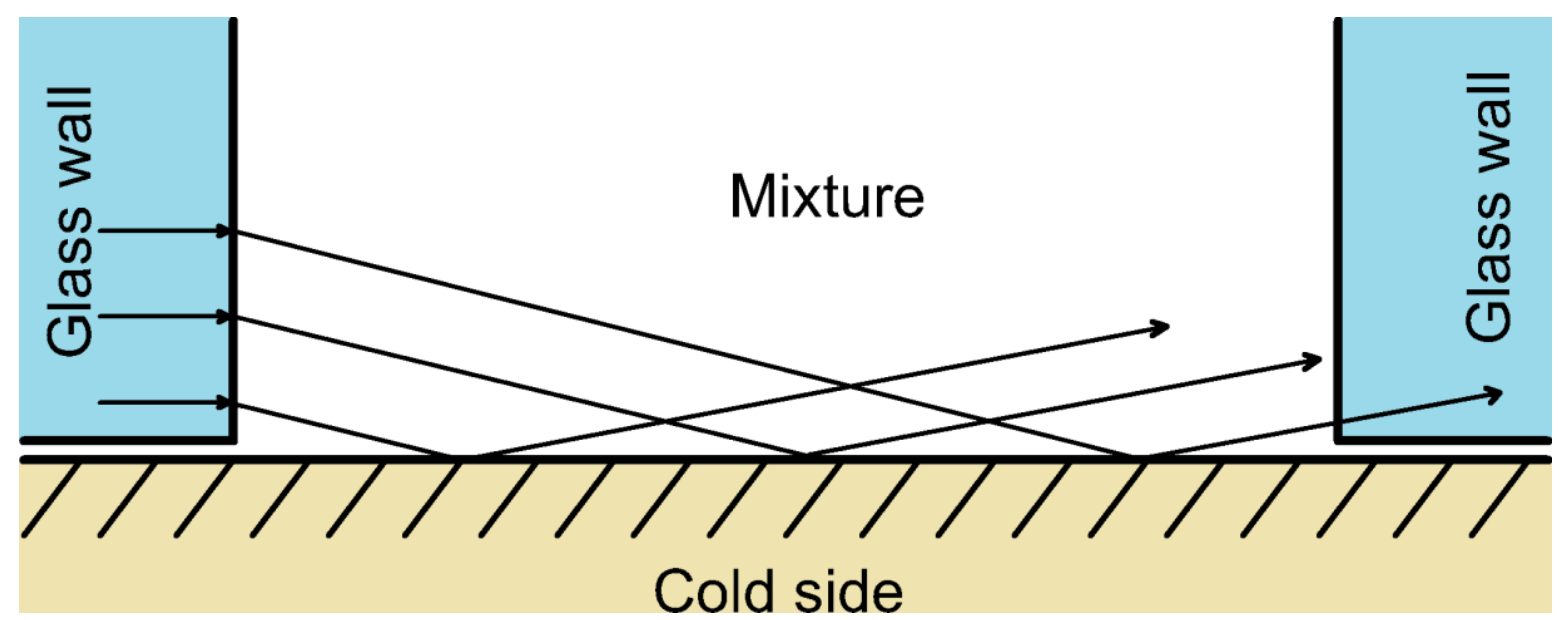

Figure 4.5: Beam deflection in a Soret cell near the cold side

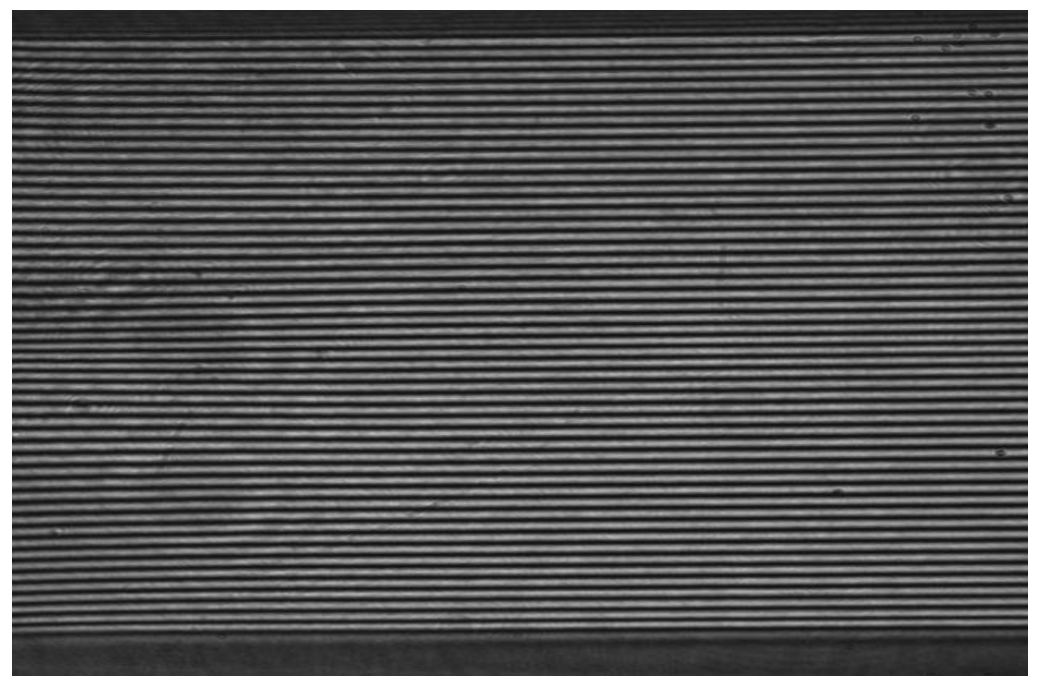

Figure 4.6: Low quality fringes near the cold side caused by beam deflection

\subsubsection{Alignment}

In case of a misalignment, the laser beam may not pass the cell completely, and the resulting fringes that come from deflections near the top and bottom of the cell are not sharp and clear (Figure 4.7). Moreover, when the incident beam is not exactly perpendicular to the cell, the assumption of constant refractive index along the traveled path is not true anymore. To level the cell, a tilt stage is used. 


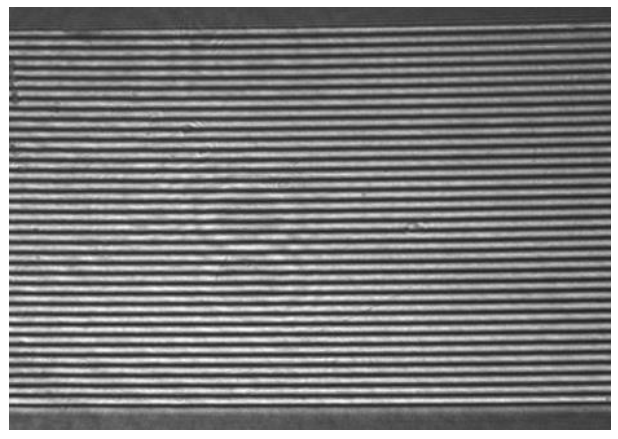

Figure 4.7: Low quality fringes near the cold side because of the misalignment

\subsection{THERMODIFFUSION- RELATED CHALLENGES}

\subsubsection{Formation of the bubbles}

The problems caused by the presence of bubbles in the cell are discussed in detail in[82], and methods to avoid the formation of bubbles are presented in the same study. In some studies a degasser is used to overcome the problem[6], while in others with a shorter height of the cell using a degasser is not necessary due to the short time of the experiment. For instance, Torres et al. [6] studied the Soret effect using a measurement time 1/10 smaller than that in [82]. Consequently, there is less possibility of the formation of bubbles during the experiment. The issue of bubble formation was faced in the current study. After trying all the known techniques to solve the problem, it was found that the best way to fill the cell was to do it from the bottom using a syringe. Then, the bubbles will exit from the top hole, and to eliminate the remaining bubbles after closing the bottom hole, the cell is refilled from the top hole using a needle syringe.

A typical bubble that appeared in the cell after installing the cell onto the Mach-Zehnder setup is depicted in Figure 4.8. As shown, a bubble can block the laser beams, and the information of that region is lost. Moreover, if a bubble shows up during the experiment in the bottom of the cell, it starts moving towards the top side and consequently an undesired flow is induced that can homogenize the mixture. Marangoni convection is another drawback of the presence of the bubbles. This effect occurs between the surface of two fluids due to the temperature and concentration gradients. 


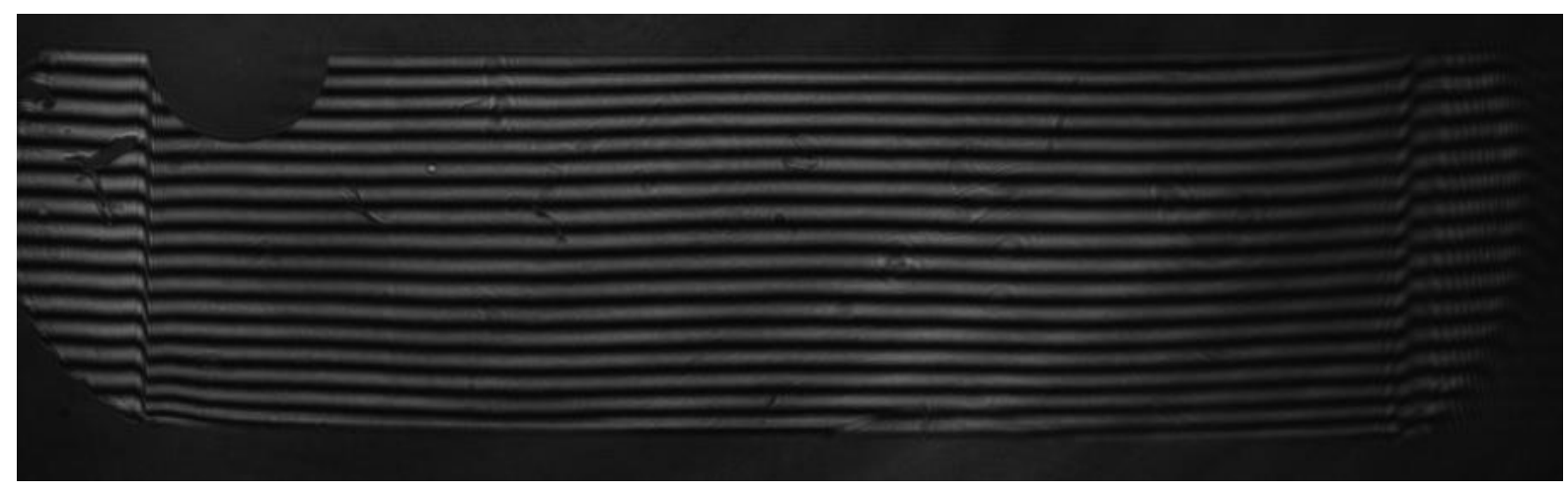

Figure 4.8: A typical bubble formed in the cell

\subsubsection{Thermal design of the cell}

Two Soret cells with different dimensions and geometries were used in the present study. Both geometries are explained here in details.

The schematic of the first cell is shown in Figure 4.9. It consists of two nickel- plated copper blocks with dimensions of $30 \times 30 \times 20 \mathrm{~mm}^{3}$ where the height is $20 \mathrm{~mm}$. A cubic cavity with quartz walls is enclosed between the two blocks. The mixture is placed in this cavity. The width and length of the glass frame are equal to those of the copper block and the height is $10 \mathrm{~mm}$. The dimensions of the cavity is $10 \times 10 \times 10 \mathrm{~mm}^{3}$. On one side of each copper block a step with height of $2.5 \mathrm{~mm}$ is provided which enters the glass frame. Thus the total height of the mixture enclosed in the cavity is $5 \mathrm{~mm}$. The aspect ratio (length/height) is two. Two concentric N-Buna O-rings with different diameters are used on each side of the frame in contact with the blocks for sealing purposes. A filling channel with diameter of $1 \mathrm{~mm}$ is provided in centre of each. After filling the cell with the mixture, blocks are sealed with screw plugs. There is a hole on side of the blocks where a thermistor enters to measure the temperature near the plate in contact with the mixture. To clamp the frame between the blocks and assemble the cell, four plastic screws are used. There is no insulation material used for this cell to prevent the heat transfer with the surrounding environment.

In the second design, the steps on the plates are removed and the glass frame is clamped between the flat plates. The size of the copper blocks is same as the previous one. The new size of the cavity is $15 \times 15 \times 2.5 \mathrm{~mm}^{3}$ where height is $2.5 \mathrm{~mm}$. So the aspect ratio increased to six. The 


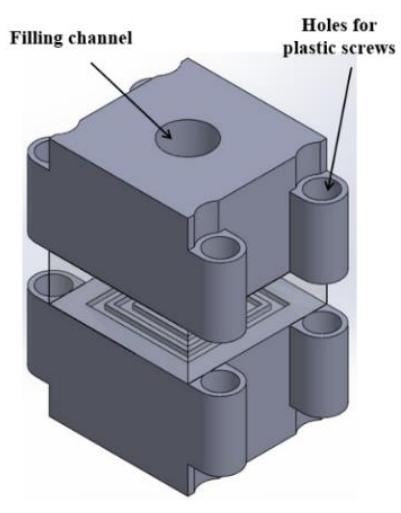

(a) isometric view

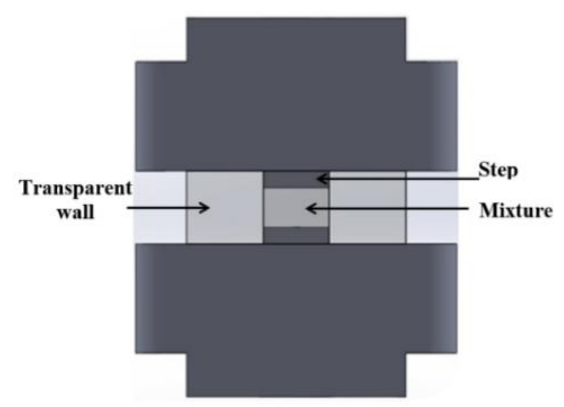

(b) side view

Figure 4.9: First designed cell

filling channels are moved to the corners of the block in order to reduce the convection effect in centre of the mixture. This will be investigated in more details in the numerical study part. Instead of using the plastic screws, PTFE Teflon walls are used to assemble the cell. The dent on top of the each block allows the PTFE wall to secure the blocks and glass frame in place. Moreover, these walls insulate the cell and prevent the heat transfer between the cell and the environment. In order to pass the laser beams, a window is provided on the front and back walls. The holes for thermistors are closer to the mixture because of removing the protrusions on the blocks. Therefore, the temperature read by the sensors is more accurate in the new design. The cross section view of the cell illustrated in Figure 4.10 shows all details of the new design.

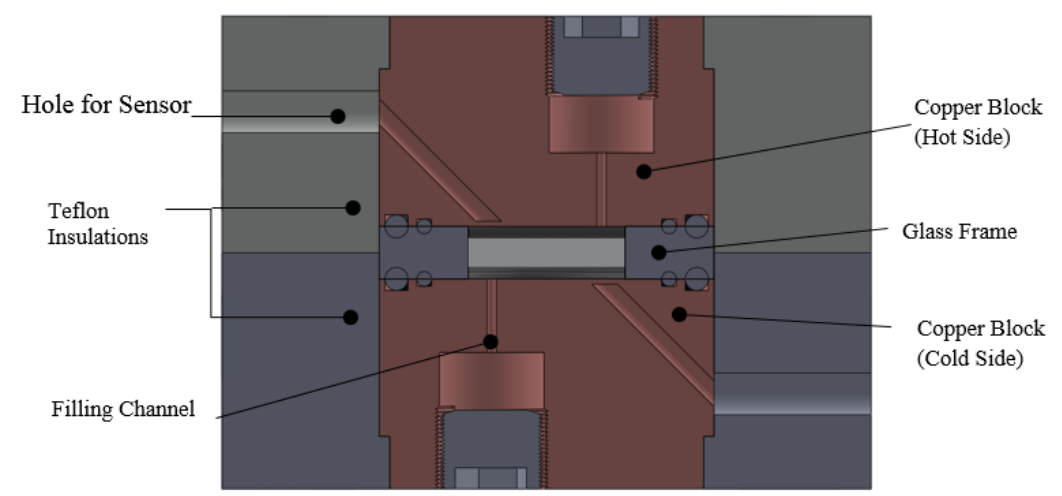

Figure 4.10: Schematic of the new design 
Peltier elements are used to thermostabilize the copper blocks. One side of the Peltier module is attached to the block and the other side is attached to the water heat exchanger. Thin layers of thermal compound is used between on the Peltier elements to increase the conductive heat transfer. The thermistor and Peltier module of each block are connected to a PID controller. Each controller regulates each Peltier. The resolution of the regulated temperature is $10^{-3} \mathrm{~K}$. The mean temperature of the experiments is $298 \mathrm{~K}$ and for the cell with height of $5 \mathrm{~mm}$ and $2.5 \mathrm{~mm}$ the applied temperature gradient is 5 and $2.5{ }^{\circ} \mathrm{C}$ respectively.

A two dimensional model was used for numerical thermal analysis in order to obtain the distribution of the temperature and velocity contours in the mixture. The both designs of the cell were examined and the results were compared. The following assumptions were made:

1- Steady state.

2- The density of the surrounding air is a function of temperature. Due to small changes in temperature, the other physical properties used in the simulation are assumed constant. The materials properties are presented in Table 4.1 [83]

$$
\rho_{\text {air }}(\mathrm{T})=3.561-1.198 \times 10^{-2} \mathrm{~T}+1.343 \times 10^{-5} \mathrm{~T}^{2}
$$

3- No phase change.

4- The gravitational effect was applied to the model vertically downward.

5- The mean temperature, $T_{\text {mean }}$, is $25^{\circ} \mathrm{C}$.

6- No thermal diffusion.

\subsubsection{Governing equations}

Based on the above assumptions, the following continuity and momentum equations were utilized in Cartesian coordinate system to calculate the velocity:

Continuity equation:

$$
\frac{\partial(\rho u)}{\partial x}+\frac{\partial(\rho v)}{\partial y}=0
$$




\section{Momentum equation:}

$$
\begin{aligned}
& \frac{\partial[(\rho u) u]}{\partial x}+\frac{\partial[(\rho v) u]}{\partial y}=-\frac{\partial P}{\partial x}+\mu\left(\frac{\partial^{2} u}{\partial x^{2}}+\frac{\partial^{2} u}{\partial y^{2}}\right)+\rho g \sin \theta \\
& \frac{\partial[(\rho u) v]}{\partial x}+\frac{\partial[(\rho v) v]}{\partial y}=-\frac{\partial P}{\partial y}+\mu\left(\frac{\partial^{2} v}{\partial x^{2}}+\frac{\partial^{2} v}{\partial y^{2}}\right)+\rho g \cos \theta
\end{aligned}
$$

where $g \sin \theta$ and $g \cos \theta$ are $\mathrm{x}$ and $\mathrm{y}$ components of the gravity respectively.

The energy equation solved to find the temperature distribution is:

\section{Energy equation:}

$$
\rho C_{p}\left(u \frac{\partial T}{\partial x}+v \frac{\partial T}{\partial y}\right)=k\left(\frac{\partial^{2} T}{\partial x^{2}}+\frac{\partial^{2} T}{\partial y^{2}}\right)
$$

In the present study, the separation of the components was studied only experimentally. In terms of the numerical investigations, thermal diffusion was not taken into account. The aim of the computational thermal analysis was to find the geometrical design with less convective motions in the mixture. Thus the mass transfer equation (Fick's law) was not included in the governing equations.

\subsubsection{Boundary and operational conditions}

The liquid is bounded on the lateral sides by the glass walls. The lower and upper boundaries of the liquid are in contact with the copper blocks. The sides of this assembly are covered by the insulation material made of PTFE. The whole cell is modelled in between a large surrounding environment with the length and height of $25 \mathrm{~cm}$ and $45 \mathrm{~cm}$ respectively. The dimension of the environment were chosen large enough so they boundaries of the environment have no effect on the natural convection around the cell.

Figure 4.11 depicts the applied boundary conditions on the model. The small objects e.g. O-rings were all modelled. However they are not shown in this figure. All the interfaces lying between a solid and a fluid zone (i.e. the interface between the mixture and the glass wall) are defined as coupled zones. In such zones the temperature of the solid and fluid are equal and there 
is no heat loss. The blue lines in Figure 4.11 represents the coupled surfaces. This type of boundary condition is described mathematically as below:

At the interface of a solid and a fluid:

$$
\begin{gathered}
\left.T(x, y)\right|_{\text {solid }}=\left.T(x, y)\right|_{\text {fluid }} \\
\dot{q}_{\text {solid }}=\dot{q}_{\text {fluid }}
\end{gathered}
$$

Constant temperature conditions were applied at the end side of each copper block:

$$
\begin{gathered}
T\left(-L_{2}<x<+L_{2}, H_{4}\right)=T_{h o t} \\
T\left(-L_{2}<x<+L_{2},-H_{4}\right)=T_{\text {cold }}
\end{gathered}
$$

The lower edge of the surrounding environment and the lateral walls are pressure inlet with the ambient temperature of $T_{a m b}(=298 \mathrm{~K})$ and the upper edge is pressure outlet with $T_{a m b}$ :

Pressure inlet condition:

$$
\begin{aligned}
& P\left( \pm L_{1}, y\right)=P_{a t m}, \quad T\left( \pm L_{1}, y\right)=T_{a m b} \\
& P\left(x, H_{1}\right)=P_{a t m}, \quad T\left(x, H_{1}\right)=T_{a m b}
\end{aligned}
$$

Pressure outlet condition:

$$
\begin{aligned}
& P\left(x, H_{2}\right)=P_{a t m} \\
& T\left(x, H_{2}\right)=T_{a m b}
\end{aligned}
$$




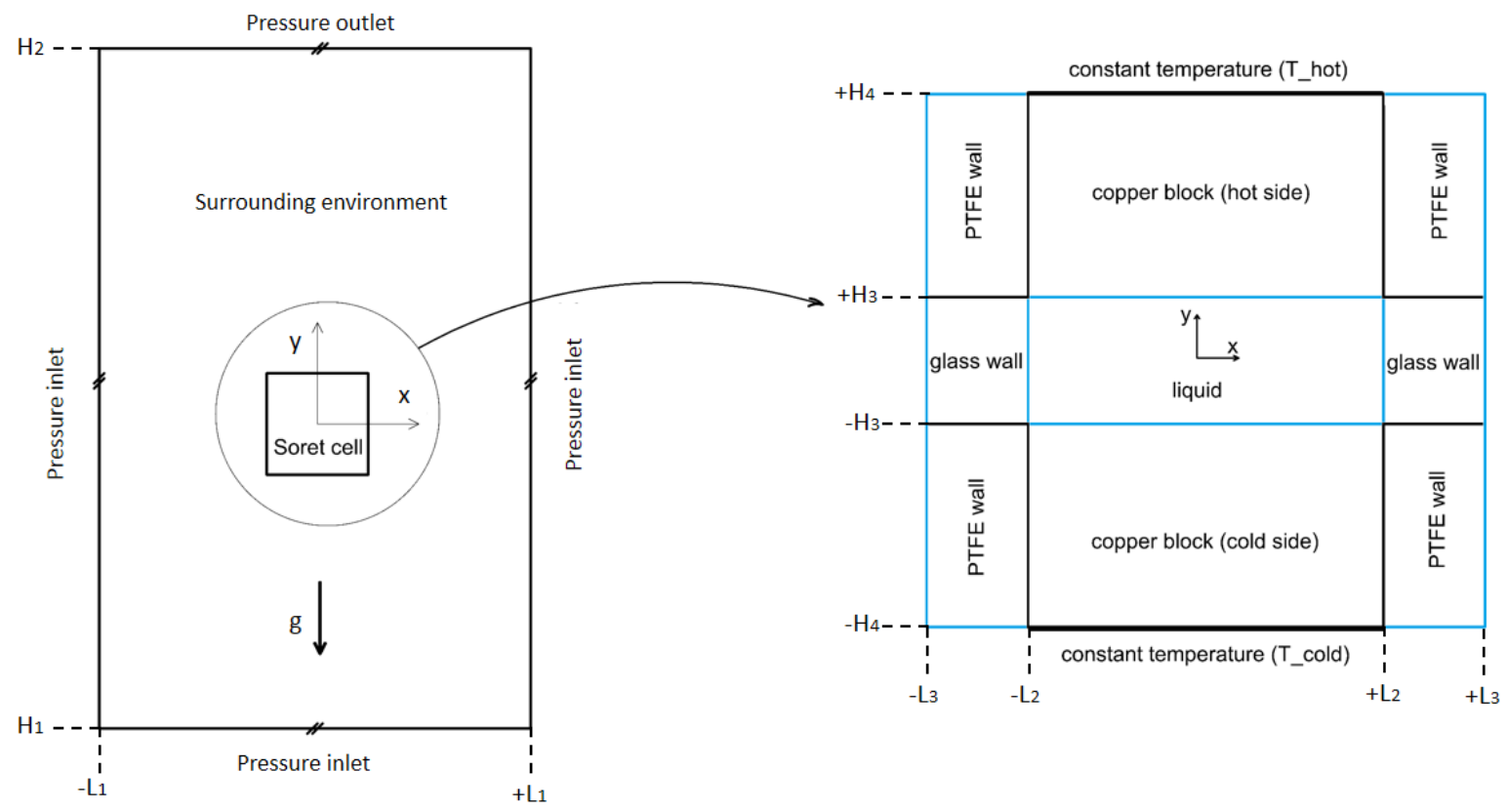

Figure 4.11 Schematic of the applied boundary conditions for the computational thermal analysis

Table 4.1: Thermal properties of materials used in numerical modelling [83]

\begin{tabular}{ccccc} 
& Density $\left(\mathrm{kg} / \mathrm{m}^{3}\right)$ & $\begin{array}{c}\text { Specific } \\
\text { heat transfer } \\
(\mathrm{j} / \mathrm{kg} . \mathrm{k}), \mathrm{Cp}\end{array}$ & $\begin{array}{c}\text { Thermal } \\
\text { conductivity } \\
\text { (W/m.k), K }\end{array}$ & Viscosity(kg.m.s), $\mu$ \\
\hline binary mixture of THN-C 12 (50/50) & 851.98 & 1851.48 & 0.13696 & 0.001763 \\
air(Fluent database) & Equation (4.2) & 1006.43 & 0.0242 & $1.79 \mathrm{E}-05$ \\
$\quad$ Glass (Quartz) & 2650 & 700 & 1.3 & - \\
PTFE (Teflon) & 2320 & 1200 & 0.25 & - \\
O-ring (Buna-n made of nitrile & 1200 & 1350 & 0.24 & - \\
rubber) & 1200 & 1000 & 1 & - \\
Gaskets (CHO_THERM1674) & 8978 & 381 & 387.6 & - \\
copper(database) & & &
\end{tabular}

\subsubsection{Numerical procedure}

Finite volume method was used to solve the governing equation. In order for the solution to converge, the residuals of mass and energy equations were considered to reach $10^{-7}$. In order to discretize the governing equations and solve the problem, a second order upwind scheme with the PRESTO! solving algorithm was used. The structured mesh used in the cavity is depicted in Figure 4.12. A finer mesh was used near the walls to be able to calculate the sharp changes in the temperature field. The grid independency analysis was performed on each model by using the 
average Nusselt number near the hot wall as the parameter for comparison. As shown in Figure 4.13, the mesh- independent solution was achieved for both models with roughly 4200 number of elements.

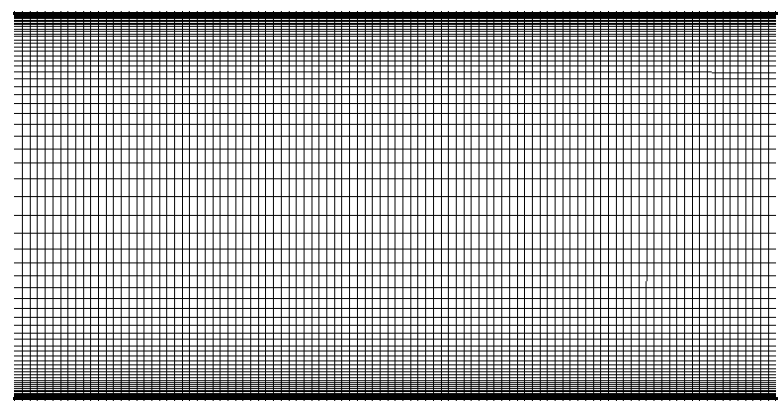

Figure 4.12: The adopted mesh in the cavity

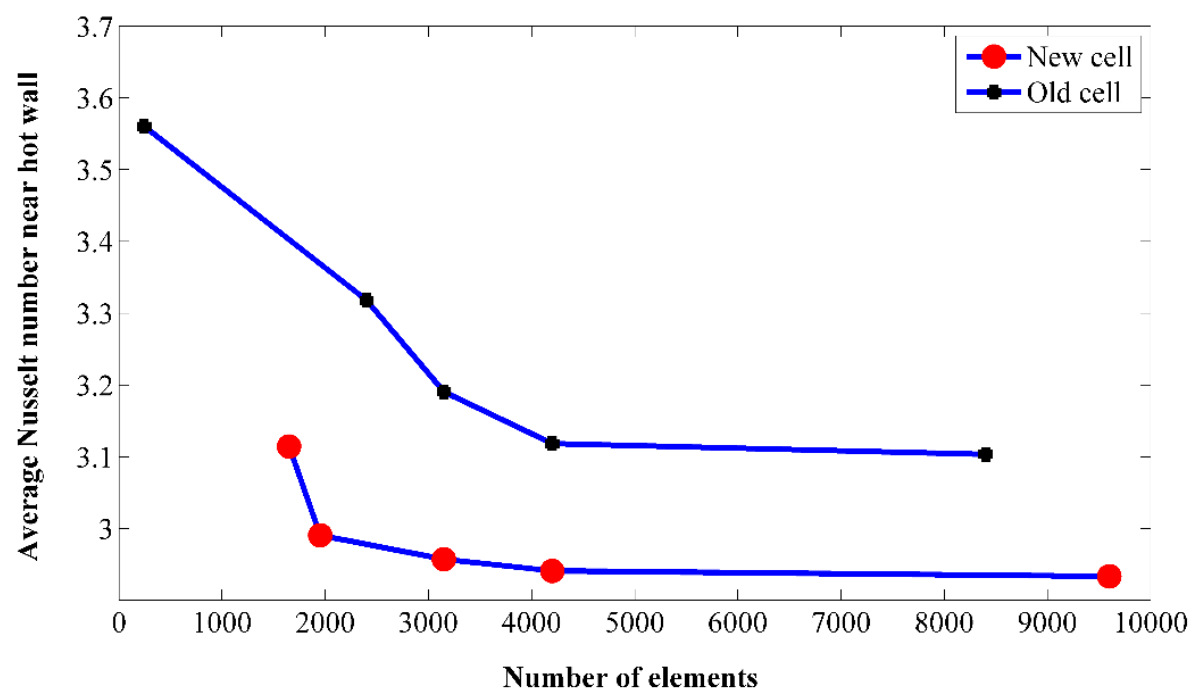

Figure 4.13: Grid sensitivity test for both models based on the average Nusselt number

\subsection{NUMERICAL RESULTS}

\subsubsection{Velocity and temperature fields in the cells}

Figure 4.14 shows the numerical computed velocity fields in the liquid contained in the cavities with different geometries. The induced motions in the cavity with smaller aspect ratio 
(height/length) is significantly greater than that for the other one. Mialdun and Shevtsova [58] showed that weak motions with velocities in range of $10-35 \mu \mathrm{m} / \mathrm{s}$ can homogenize back the mixture and prevent the segregation of the components. The average and maximum velocities are presented in Table 4.2. Although the model shown in Figure 4.14 (a) experiences velocities smaller than $2 \mu \mathrm{m} / \mathrm{s}$, the second model has even smaller velocities less than $0.06 \mu \mathrm{m} / \mathrm{s}$.

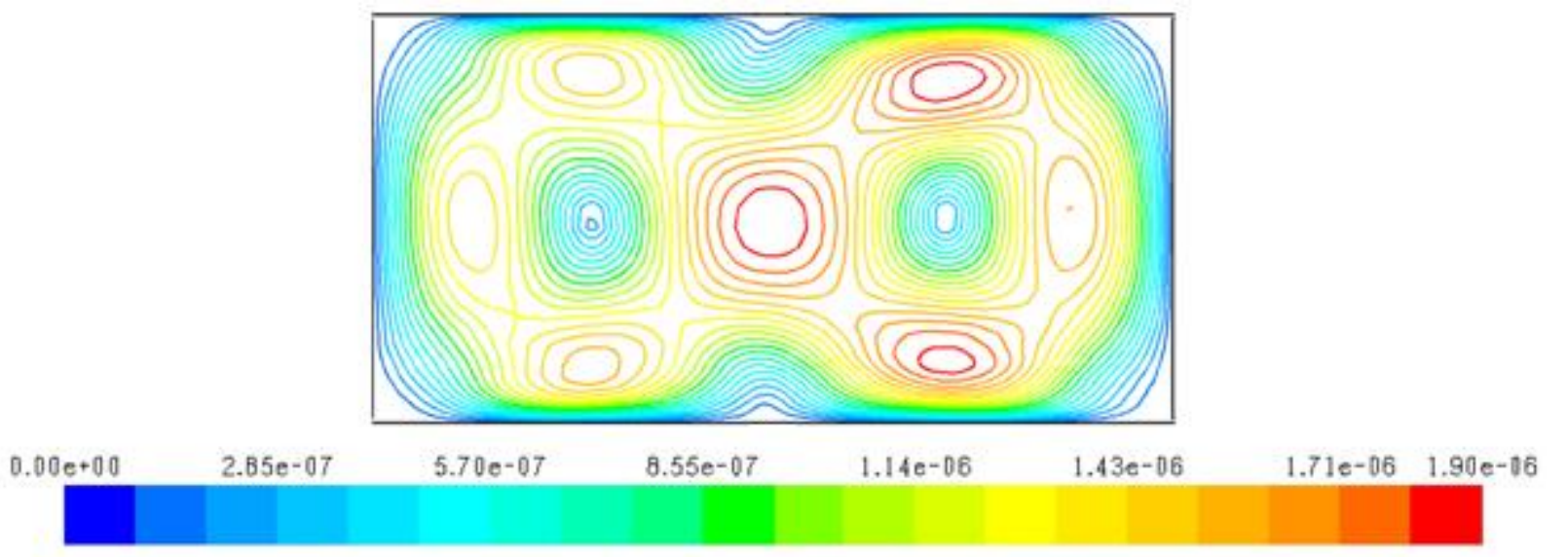

(a) aspect ratio $=2$
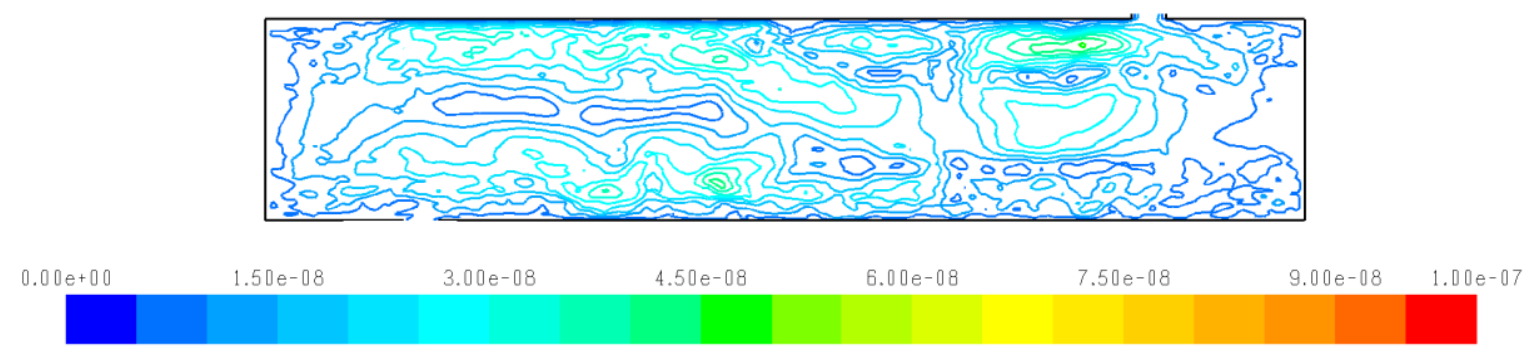

(b) aspect ratio $=6$

Figure 4.14: Computed velocities in different geometries

Table 4.2: Maximum and average velocities in the mixture (numerically computed)

New cell (aspect ratio $=6) \quad$ Old cell $($ aspect ratio $=2)$

$\begin{array}{ccc}\text { Average velocity } & 1.42 \mathrm{e}-8 \mathrm{~m} / \mathrm{s} & 1.015 \mathrm{e}-6 \mathrm{~m} / \mathrm{s} \\ \text { Maximum velocity } & 4.85 \mathrm{e}-8 \mathrm{~m} / \mathrm{s} & 1.898 \mathrm{e}-6 \mathrm{~m} / \mathrm{s}\end{array}$


The computed steady isotherms are depicted in Figure 4.15. The temperature gradient of $\Delta T=1 \mathrm{~K} / \mathrm{mm}$ was applied against the gravity between the top and bottom walls. Thus $\Delta T$ for previous and new model is 5 and $2.5 \mathrm{~K}$ respectively. The temperature gradient in horizontal direction exists near the side walls for both models with smaller values for the shorter cell. Moving towards the center of the cells the horizontal temperature gradient disappears. However, sudden changes in isotherms are observed at the connection point between each of the filling channels and the cell. As in the new model these channels are moved to the corners, the horizontal temperature gradient in center of the cell still remains zero which is beneficial to establishing a motion free zone in center zone. As a drawback for the previous cell, the horizontal temperature gradients reside at the corners as well as the center of the cell and the assumption of pure heat conduction is not applicable and convection is inevitable. The temperature distribution on a horizontal line near the wall shown in Figure 4.16 illustrates the mentioned jumps in temperature at the connection point.

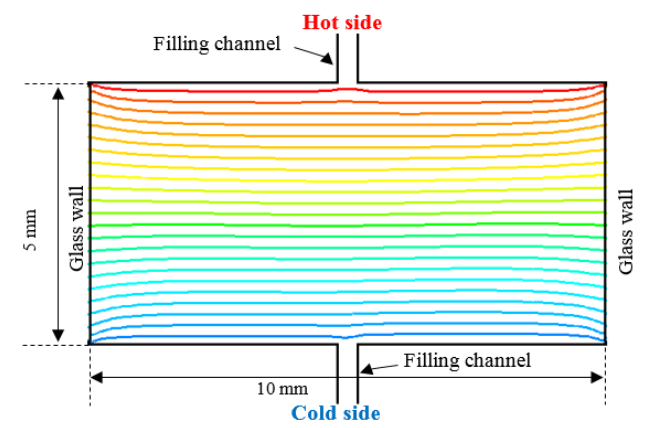

(a) previous model

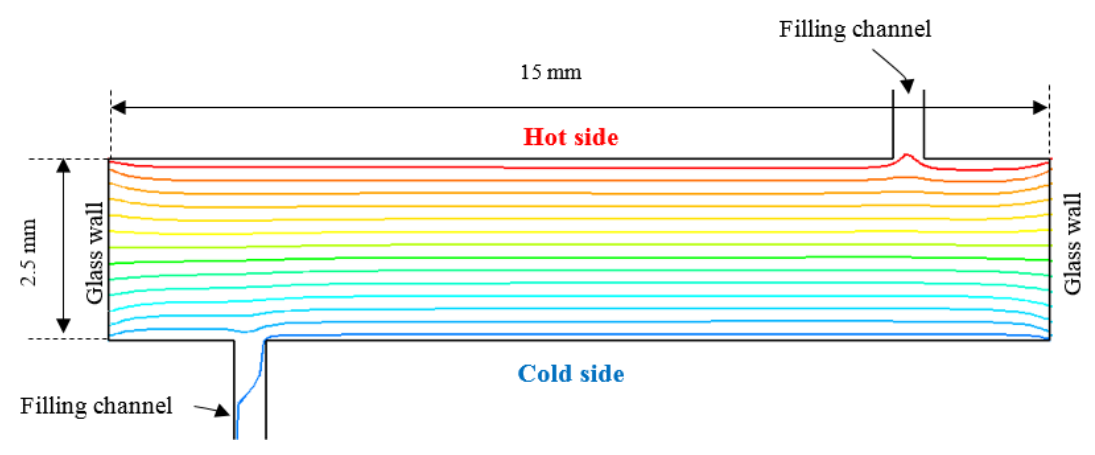

(b) new model

Figure 4.15: Numercally computed isotherms 


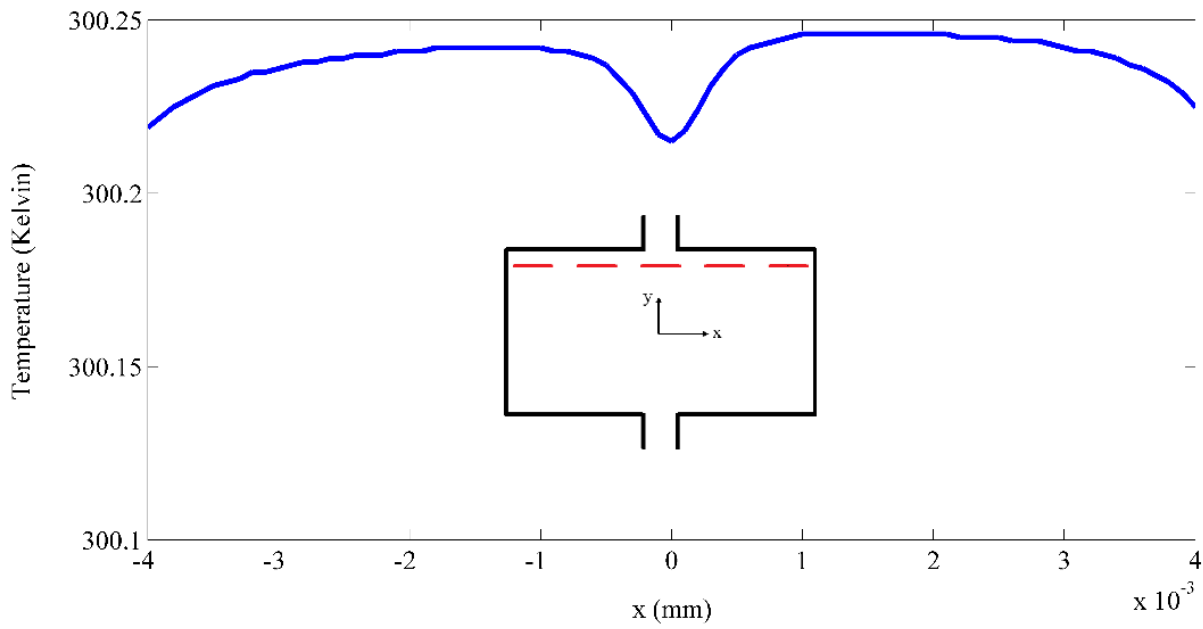

(a) previous model

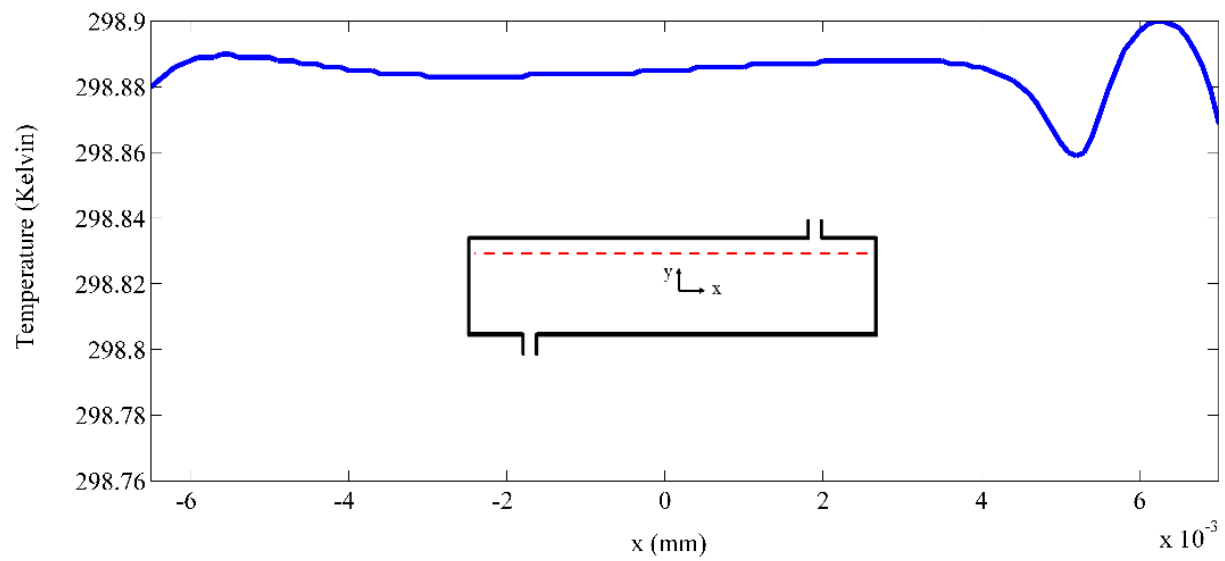

(b) new model

Figure 4.16: Temperature distribution on a horizontal line near the hot wall

The linearity of the temperature distribution on a vertical line for two models is examined in Figure 4.17. The insertion shows the temperature near the hot wall. The blue lines represent the numerical results and the red lines are linear fitting to the computed results. In agreement with the results of Figure 4.15, the temperature profile in the new model is completely linear which indicates the pure heat conduction. 


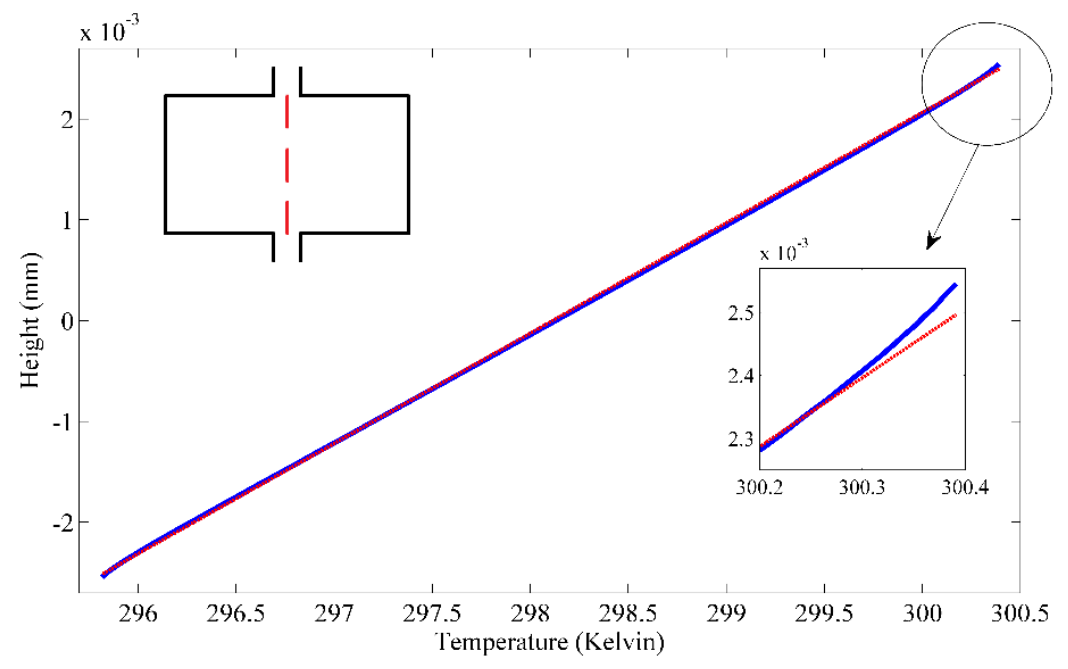

(a) previous model

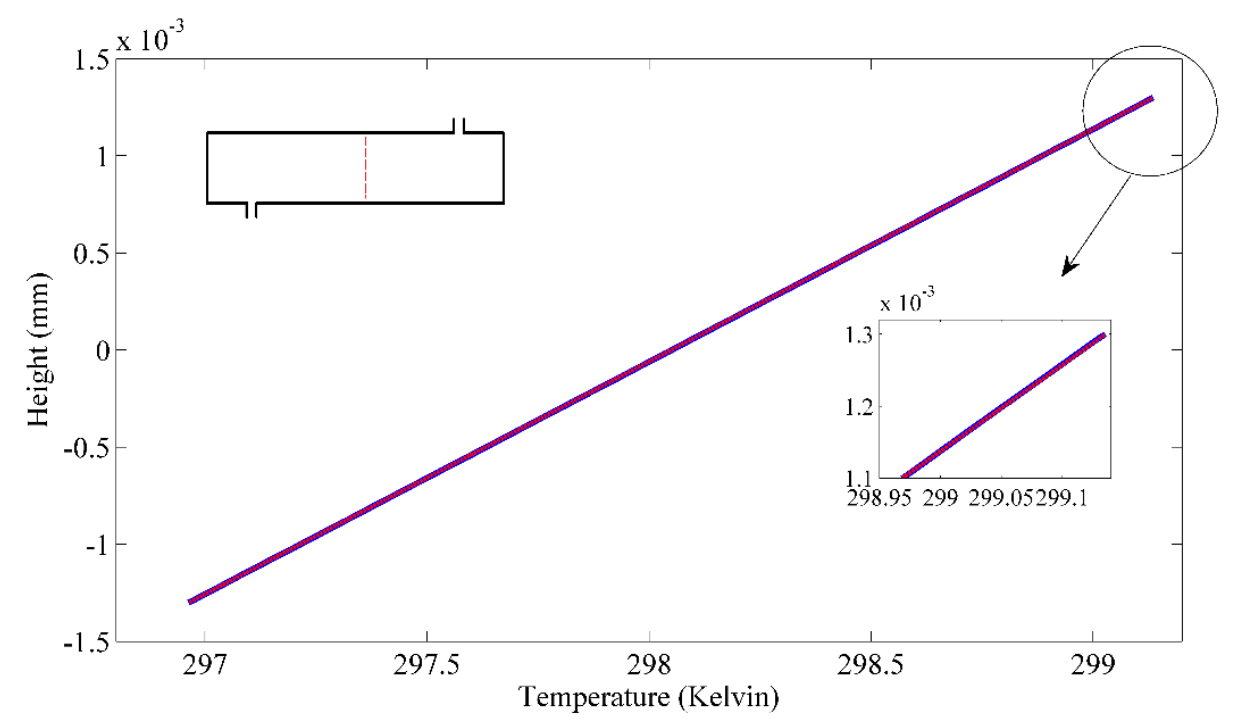

(b) new model

Figure 4.17: Temperature profile on a vertical line between hot and cold sides of the cell

\subsubsection{Effect of inclination}

Figure 4.18 illustrates an inclined cell. The incident beam is perpendicular to plane $x y$ and parallel to $z$ axis. Different angles of inclination were tested. Figure 4.19 shows the velocity 
contour in different inclination of the cell computed numerically. In all cases convection is considerably more pronounced than in case of no inclination. Because of the no-slip condition, the fluid has zero velocity on the walls. By moving towards upper and lower walls velocity increases. For angles between zero and 2.5 degree the maximum velocity in the cell occurs close to the right lateral wall. However, in the case of $\theta=5^{\circ}$, the maximum value of the velocity happens in the upper region of the central point. In all cases, the motion-free zone is located almost in centre of the cavity. By increasing the inclination of the cell, the area of the motion-free zone diminishes.

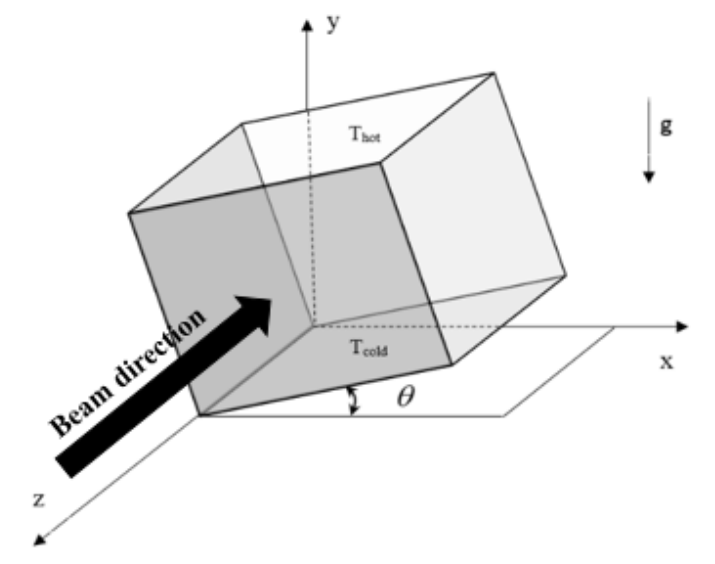

Figure 4.18: Tilted cell

Figure 4.20 shows the velocity profile on a vertical line in middle of the cell for different angles. Even 0.5 degree of inclination causes a noticeable increase in velocity and it means that installation must be done very carefully to avoid any inclination and the cell must not be tilted at all. However, as mentioned earlier, the weakest region in term of the velocity is located in the centre of the cell. The isotherms established in the mixture for different inclinations are illustrated in Figure 4.21. In case of no inclination, the isotherms are almost straight and horizontal. However, the isotherms are curved at the points of the connection of the cavity and the filling channels. These curvatures of the isotherms are observed at upper right and lower left of the cavity.

By increasing the inclination, the horizontal shape of the isotherms is disturbed and a temperature gradient in $\mathrm{x}$ direction is established. This effect is more profound near the lateral walls. This temperature gradient near the lateral walls leads to heat transfer between the mixture and the quartz frame. The lateral heat fluxes generate more convection near the walls. Moreover, 
the velocity contours confirm the fact that in the inclined cell the convection is more significant near the lateral walls.

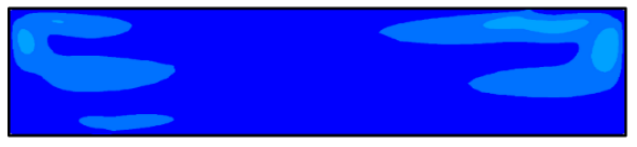

$\theta=0$

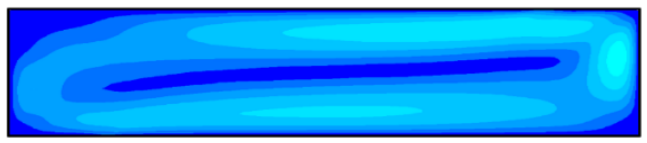

$\theta=1^{o}$

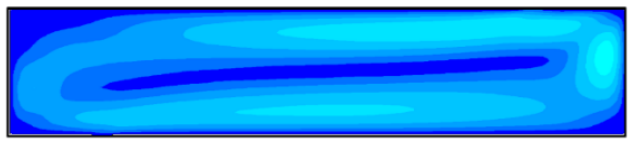

(b) $\theta=0.5^{\circ}$

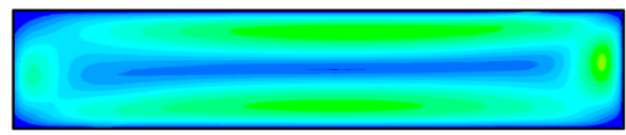

$\theta=2.5^{\circ}$

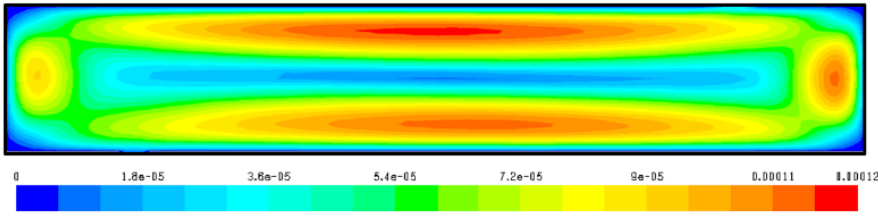

$$
\theta=5^{\circ}
$$

Figure 4.19: Velocity contour in the cell with different inclinations (a)Theta $=0$, (b) Theta $=0.5$, (c) Theta=1, (d) Theta=2.5, (e) Theta=5 degree

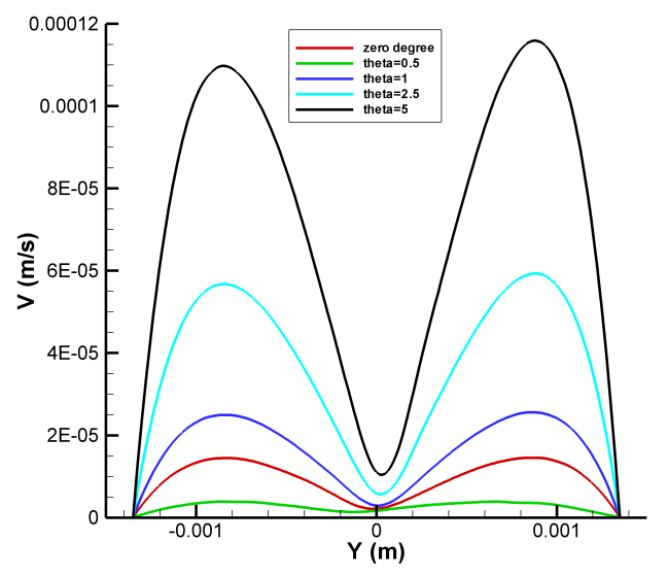

Figure 4.20: Velocity on center line for different inclinations of the cell 


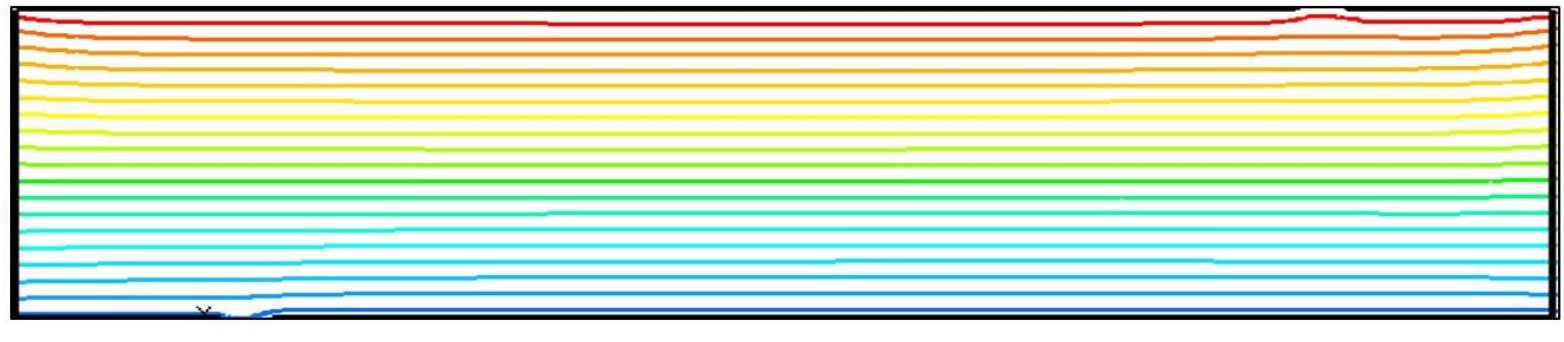

$$
\theta=0
$$

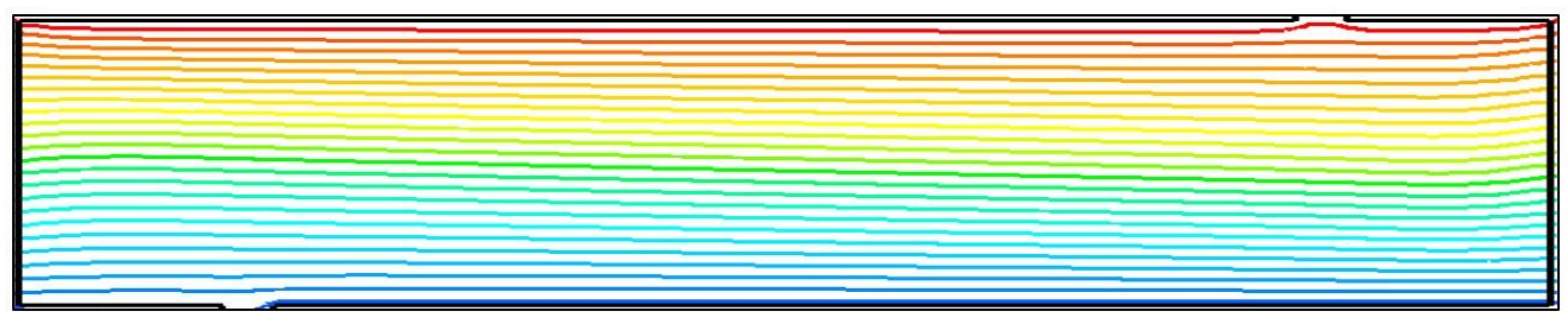

$$
\theta=2.5^{\circ}
$$

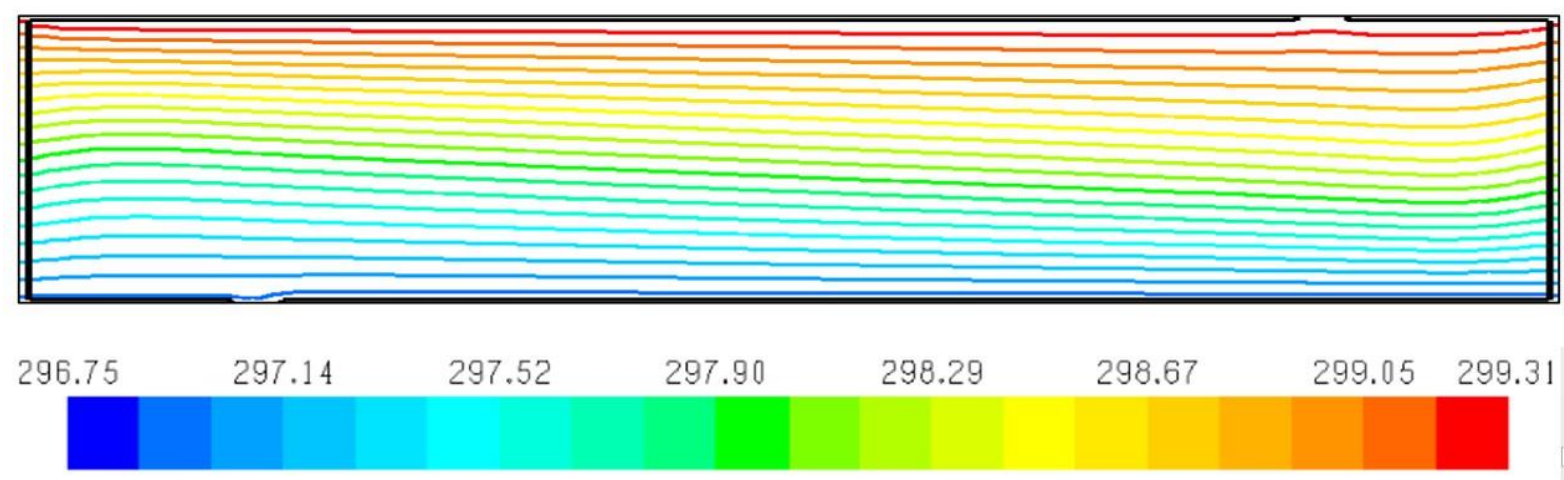

$$
\theta=5^{\circ}
$$

Figure 4.21: Isotherms in the mixture in different inclination

\subsection{EXPERIMENTAL RESULTS}

The experimentally measured concentration distribution in the previous cell is shown in Figure 4.22 (a). Note that the vertical dark lines are defects of the image processing and the actual contour does not include such lines. Significant disturbed regions are detected in the corners which indicates noticeable vortexes of motion. The vertical concentration profile at centre of the cell is plotted in Figure 4.22 (b). As seen, there is only a small region of linear concentration distribution. This region can be addressed as the motion free zone. However this is dramatically small and can 
be affected by the surrounding convective motions. The vertical temperature profile shown in Figure 4.23 (a) with solid blue line is disturbed near the hot and cold sides. These deviations from the linearity reveals convections which are more profound near the top and bottom boundaries. The linearity of the temperature profile of new cell and the corresponding concentration distribution shown in Figure 4.24 confirmed this statement that by having a more linear temperature profile, weaker convections must be experienced.

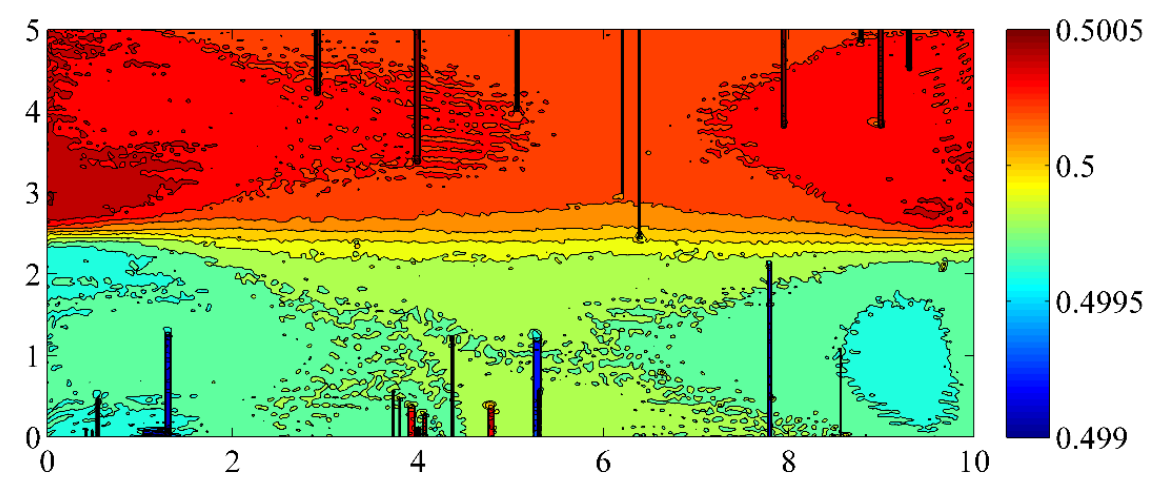

(a) distribution in the whole cell

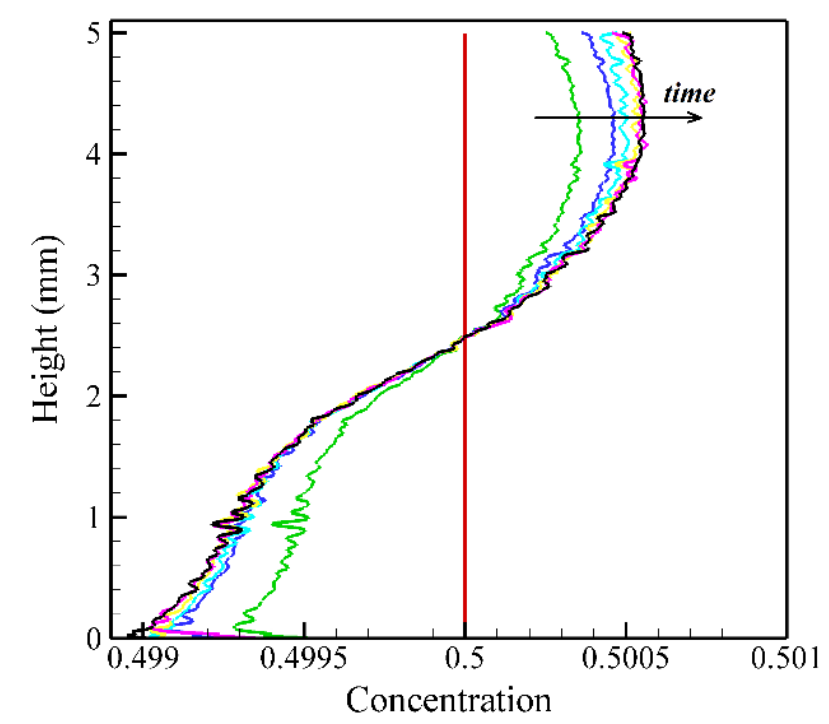

(b) distribution on a vertical lie in centre of the cell

Figure 4.22: Concentration field in the previous design 


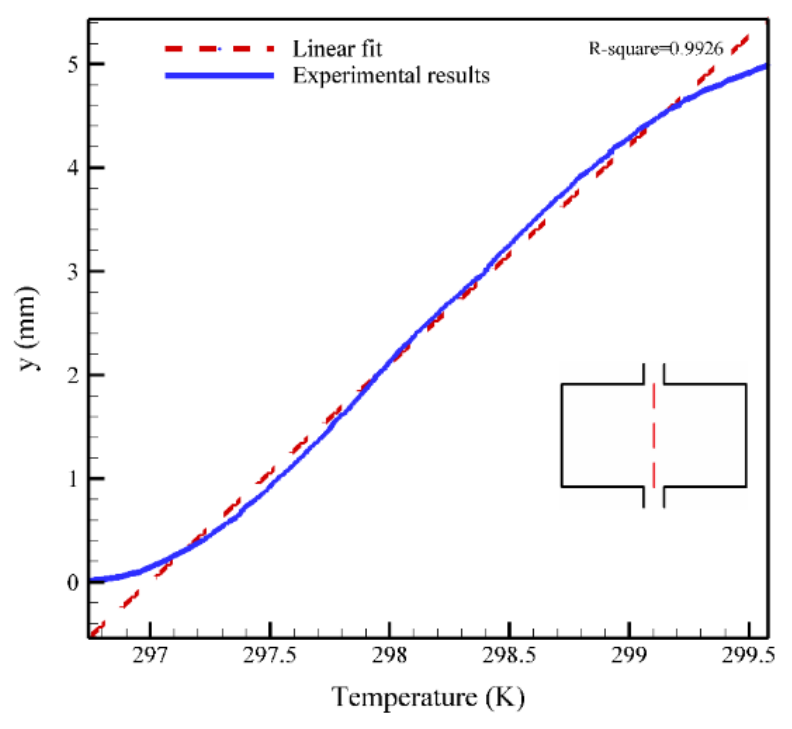

(b) previous design

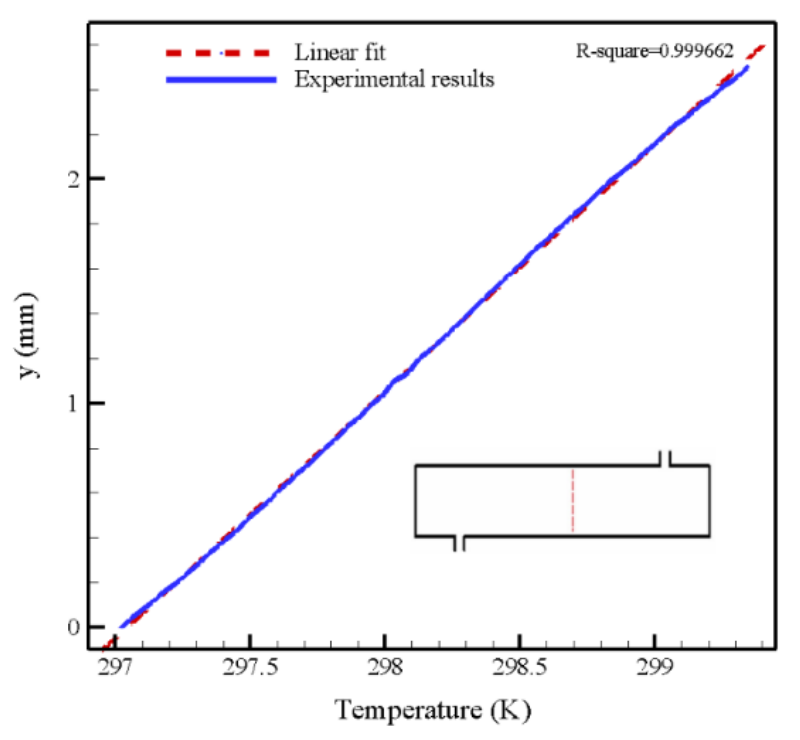

(b) new design

Figure 4.23: Temperature profile 


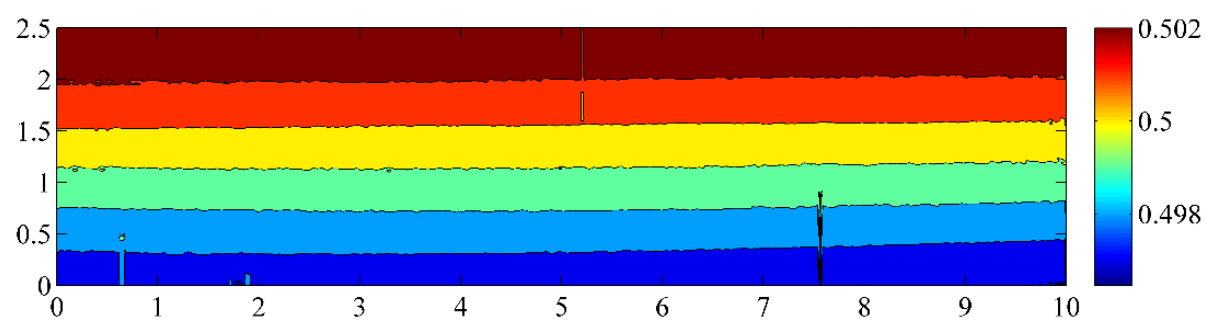

(a) distribution in the whole cell

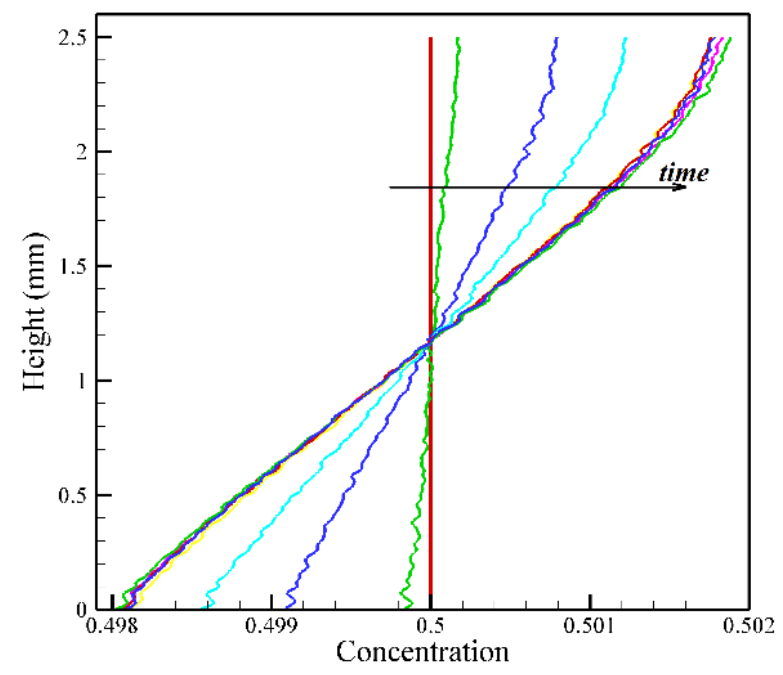

(b) distribution on a vertical lie in centre of the cell

Figure 4.24: Concentration field in the new design

\subsubsection{Soret coefficient, $S_{T}$}

The evolutions of the concentration difference between the hot and cold sides (i.e. the maximum separation) are shown in Figure 4.25. The cell with height of $5 \mathrm{~mm}$ reaches the steady state after about 600 minutes. By decreasing the height of the cell the diffusion characteristic time decreases based on Equation (4.13).

$$
\tau_{D}=H^{2} / D
$$

where $H$ is height of the cell and $D$ is diffusion coefficient which is constant. The system with the shorter cell becomes steady after 200 minutes. The experimental results are tabulated in Table 4.3. The results of the new design are in a great agreement with the values of the benchmark [60] with standard deviation of $5.8 \%$ while the previous cell has results smaller than the 
benchmark's. Due to the strong convective motions in the previous model, the separation of the components was not done properly while in the new design the problem of convection was solved and separation occurred without the interference of the residual convections.

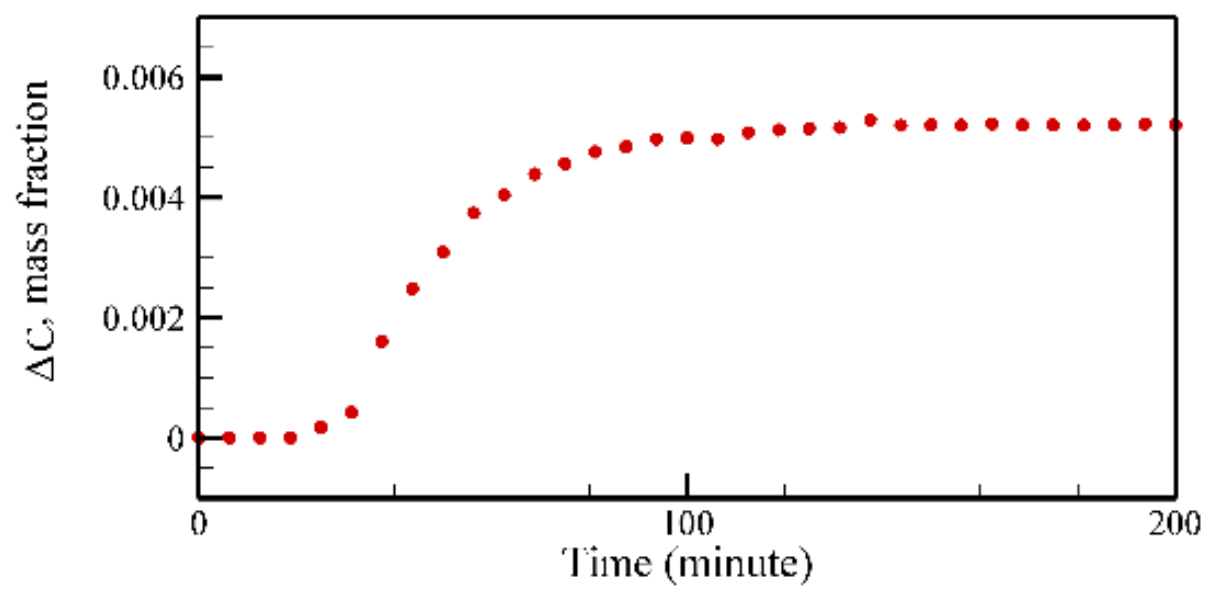

(a) previous design

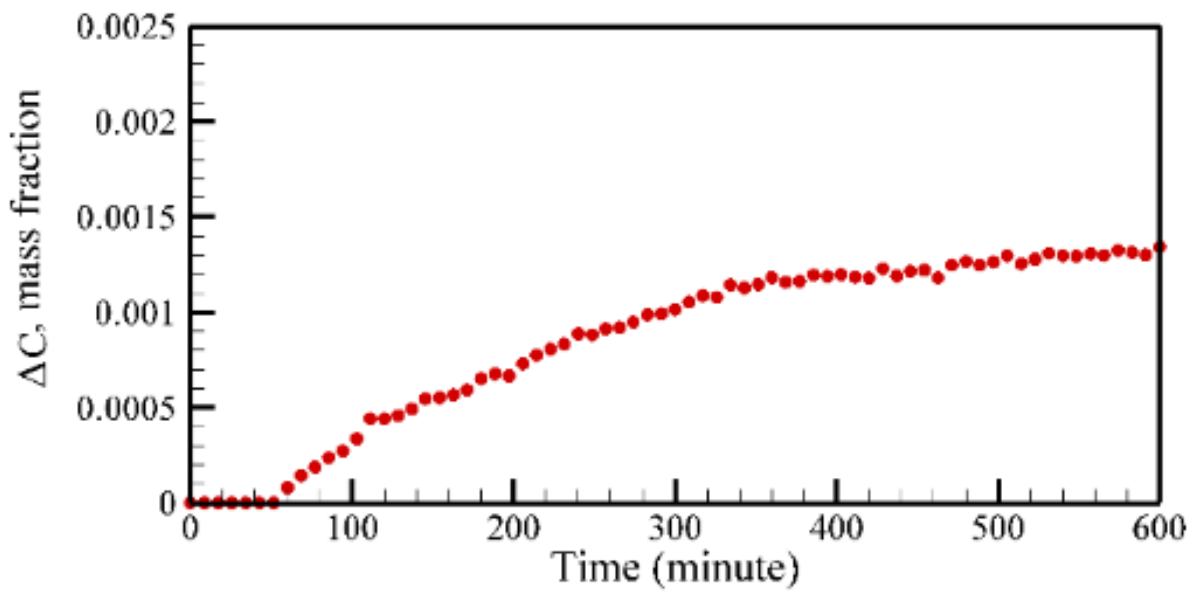

(b) new design

Figure 4.25: Evolution of maximum separation 
Table 4.3: Experimental results

\begin{tabular}{cccc}
\hline cell & $\Delta C_{\max } / 10^{-3}$ & $\Delta T$ & $S_{T} / 10^{-3}\left[\mathrm{~K}^{-1}\right]$ \\
\hline previous design & 1.49 & 5.06 & $1.09 \pm 0.16$ \\
new design & 5.43 & 2.33 & $9.32 \pm 0.1$ \\
\hline
\end{tabular}

\subsubsection{Effect of inclination}

Six different degrees of inclination were studied: zero (aligned horizontally), 0.5, 1, 1.5, 2, and 2.5 degree.

As shown in Figure 4.24 (a), in a horizontal cell, the concentration varies with the height. By performing the experiment with an inclined cell, the horizontal nature of the layers is disturbed. Figure 4.26 depicts the steady concentration field after thermal diffusion time for different angles of inclination. As seen, by increasing the angle of inclination, the layers of the concentration lose their horizontal nature.

Figure 4.27 illustrates the concentration profile along the height of the cell for different inclinations of the cell. By increasing the degree of the inclination, the linearity of the concentration decreases which means the raise in the convection residuals. Thus, the assumption of the pure conduction cannot be applied to the problem anymore and the Soret coefficient is not defined for the case. In conclusion, an inclined cell cannot be used to measure the Soret coefficient and aligning the cell for the Soret experiment is crucial. 


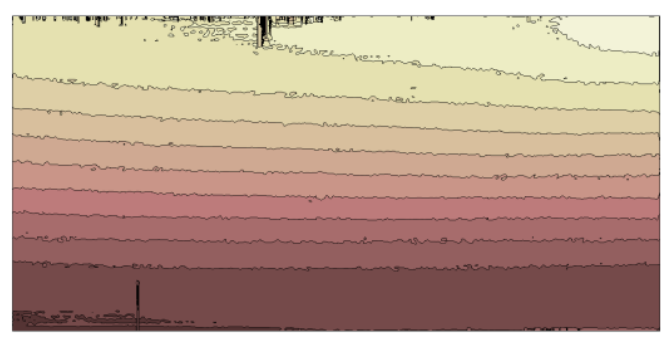

(a) half

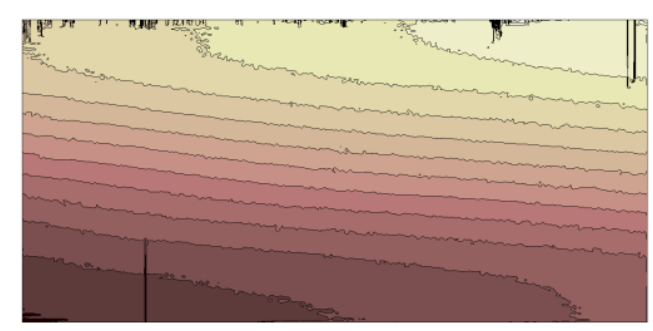

(c) 2 degree

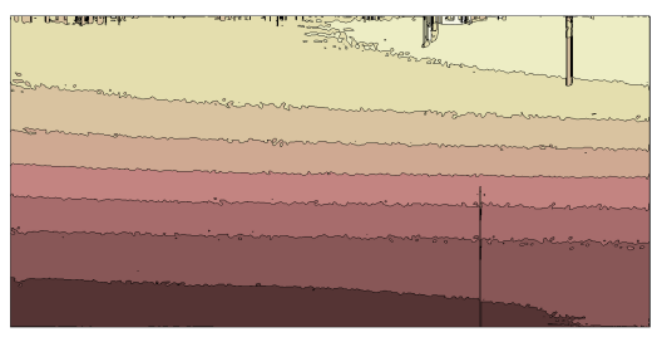

(b) 1 degree

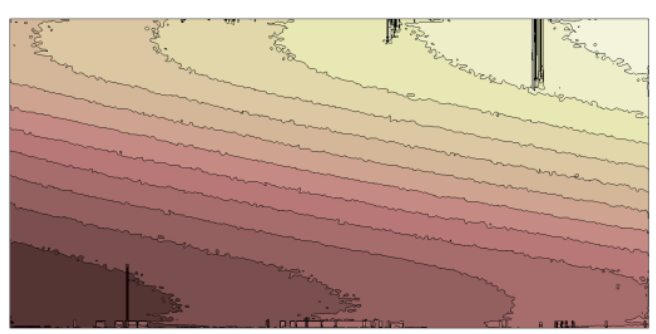

(d) 2.5 degree

Figure 4.26: Concentration contour at different inclinations of the cell

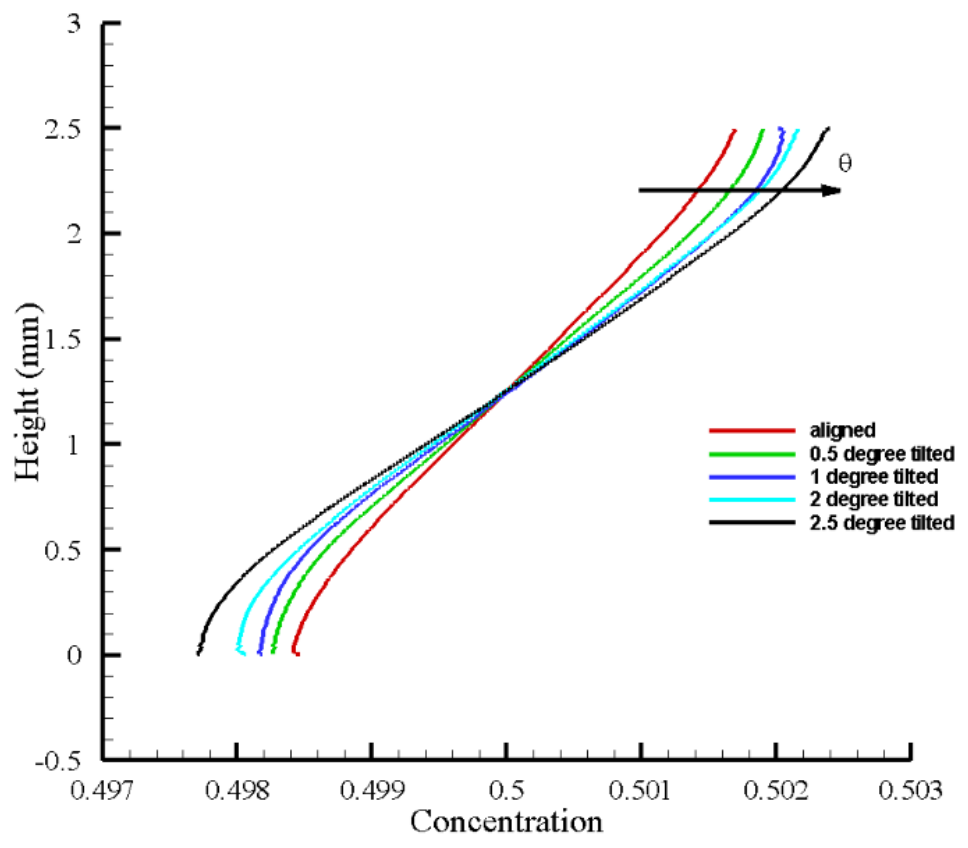

Figure 4.27: Concentration profile at different inclinations of the cell 


\subsection{CONCLUSIONS}

Optical interferometry technique with MZI arrangement was used to measure thermal diffusion in binary hydrocarbon mixture composed of THN and $\mathrm{C}_{12}$ at the mean temperature of $25^{\circ} \mathrm{C}$. The thermal design of the cell filled with the mixture was investigated numerically and

finally, for the first time, the effect of the inclination of the cell on the segregation of the components was studied.

In the optical interferometry techniques, beam deflection is a deleterious effect and must be avoided. By increasing the gradient of the refractive index the beam deflection rises. Thus, this error increases by rising the temperature gradient and increasing the optical path in the cell. It was experimentally shown that by adjusting the focal plane of the plano-convex lens on the inner side of the glass wall near the CCD camera, the beam deflection problem can be minimized. Another interferometry-related factor that could cause errors in measurement is the angle between the cell and the incident beam. This angle must be $90^{\circ}$ always. If the beam was not perpendicular to the cell, then the assumption of constant refractive index along the beam would not be true anymore. A tilt stage along with a rotary stage are used to adjust the cell perpendicular to the incident beam.

Formation of the air bubbles during the experiment disturbs the experiment and the separation process is prevented by the Marangoni convection. In the new design of the cell with flat copper blocks and degassing the mixture from upper filling channel, no more bubble was formed and the problem was fixed. The new design of the cell established more linear temperature and concentration profiles compared to those formed in the previous design. In result the measured Soret coefficient was in agreement with the benchmark values within the experimental errors.

Finally, it was found that by tilting the cell the convective motions grow which is undesirable in convectionless experiments. Thus it is essential to align the cell completely horizontal without any inclination in order to measure thermal diffusion properly. 


\section{DATA PROCESSING AND ERROR ANALYSIS FOR BINARY AND TERNARY MIXTURES}

\subsection{INTRODUCTION}

In the Soret convectionless experiments, external mechanical forces must be avoided. Interferometry is a non-intrusive technique and it does not include use of any moving parts, which does not add to mechanical forces on the measurement cell. In addition, by using this technique, transient thermal diffusion can be studied and the temperature and concentration profiles in the entire cell are obtained. Because of these advantages, the Mach-Zehnder arrangement of interferometry was chosen as the experimental method of the present study. Briefly, two coherent light waves (the reference beam and the object beam) overlap, and a fringe pattern is produced. In the Mach-Zehnder arrangement, the reference beam passes through the undisturbed environment, while the object beam passes through the test cell. Due to the phase difference between the object and reference beams, a periodic intensity pattern called a fringe pattern, is established and captured by the CCD camera.

The refractive index of the working liquid mixture varies with changes in the concentration and temperature of the mixture. The change in the index of refraction causes a phase change in the beam, which appears as fringes. For instance, Figure 5.1(a) illustrates a fringe pattern of a cell with sides at the same temperature of 25 degrees Celsius, and Figure 5.1(b) depicts the fringe pattern after establishing a temperature gradient of 2.5 degrees Celsius between the top and bottom sides of the cell. The thickness of the fringes decreased by applying the temperature gradient and the number of fringes increased. By increasing the temperature difference, the fringe spacing decreases. This decrease in the thickness of the fringes is also happen by increasing the optical path difference between objective and reference beam e. g. by increasing the length of the cell.

The next step is to analyse the fringe patterns and extract the phase information. There are different techniques available for image processing. However, the two most important methods are two-dimensional fast Fourier transformation (2D-FFT) and phase-shifting. The accuracy of 2DFFT is between $2 \pi / 50$ and $2 \pi / 100$. Mialdun and Shevtsova [65] developed an Interferometric 
technique along with an FFT image processing method to measure the Soret coefficients of hydrocarbon mixtures and named it optical digital interferometry (ODI). Later on, Torres et al. [6] applied a phase-shifting method to enhance the precision of calculating the phase shifts of the fringe patterns. In the present study, a 2D-FFT technique has been chosen in order to process the images and calculate the temperature and concentration profiles, and with further calculation the Soret coefficient was obtained. Figure 5.2 depicts the steps of the fringe processing used in this study.

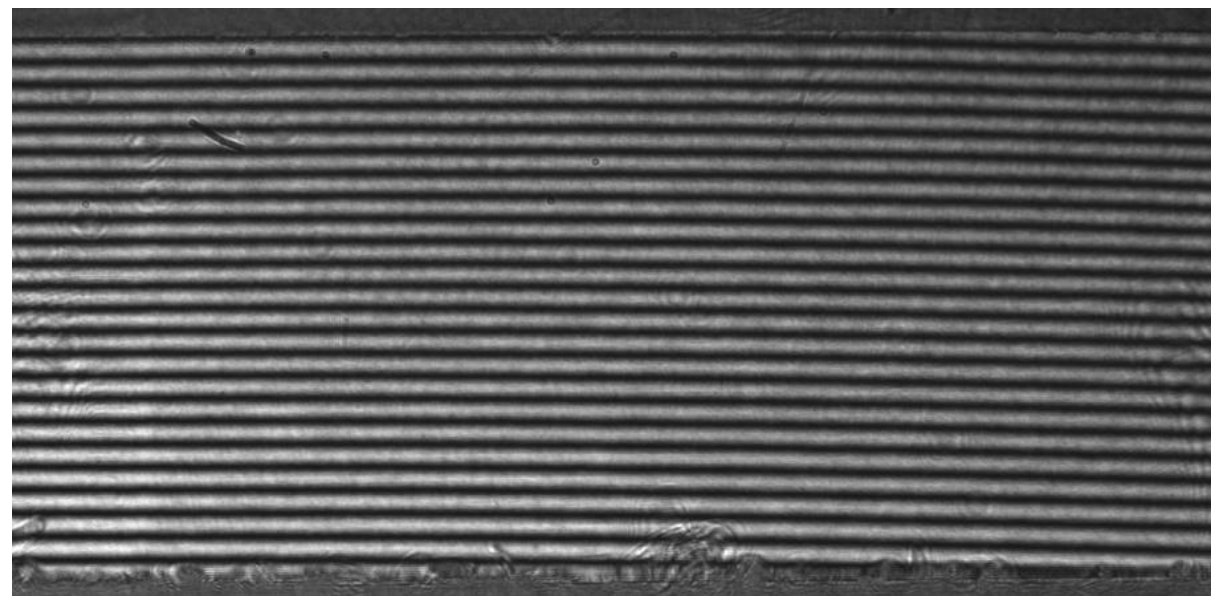

(a)

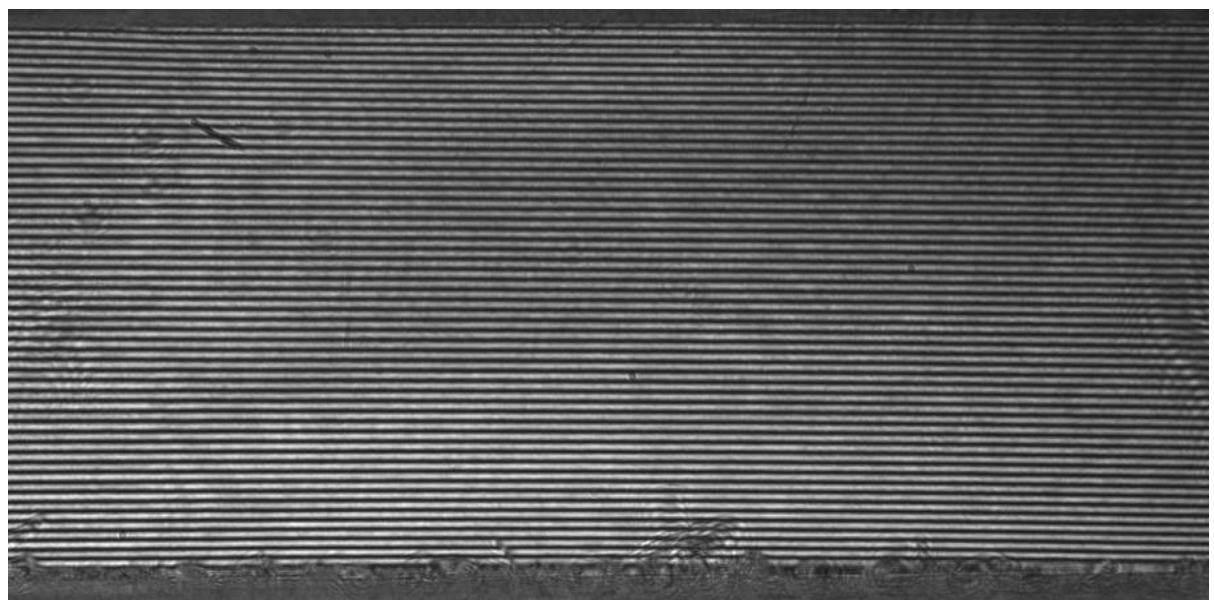

(b)

Figure 5.1: Fringe pattern in the mixture established because of optical path difference between Mach-Zehnder arms: (a) no temperature gradient between the sides, (b) 2.5 degree Celsius difference between the top and cold side. 


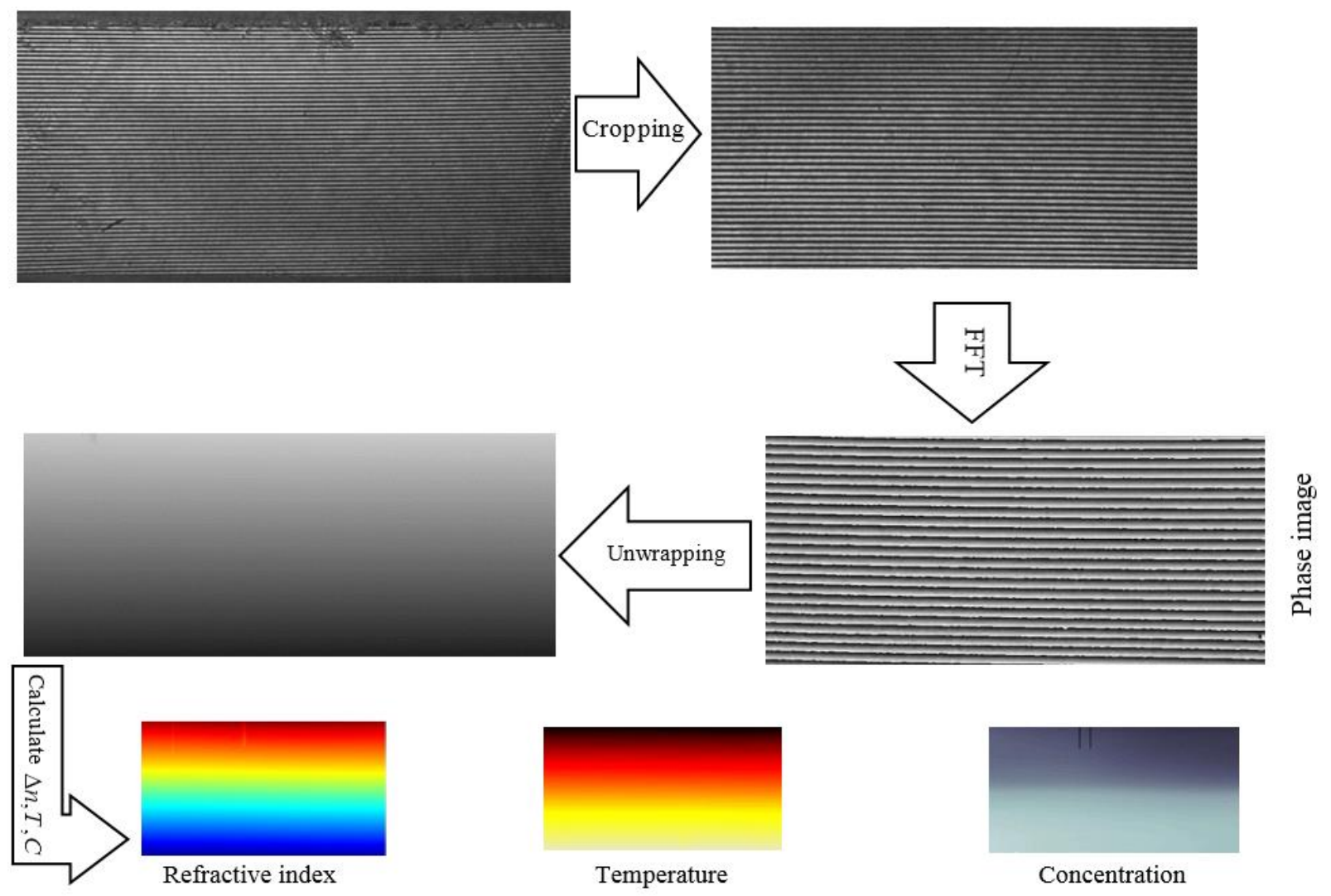

Figure 5.2: Extracting information for the fringe patterns using FFT method

Rahman and Saghir [78] used ODI for ground based measurement of the Soret coefficients of binary hydrocarbon mixtures and compared the results with the values of Fontainebleau benchmark. The error bar of the Soret coefficient corresponding to the binary mixture of THN-C 12 is \pm 0.5 . The method of root sum square (RSS) was used in [78] to calculate the error. The same method is used to find the error value in ternary systems.

\subsection{ChaPTER AIMS}

The main goal of the present chapter is to describe the method used to extract temperature and concentration fields from the fringe patterns. The different steps of the image processing for 
the binary and ternary mixtures is discussed in details. The subtraction of the images to cross out the undesirable phase shifts is presented. Finally, the uncertainty analysis is explained.

\subsection{Data ANALYsis}

Light has the fastest speed in the vacuum while it slows down passing through materials. Refractive index or index of refraction is used to show that how much light is refracted in a specific medium and is defined as:

$$
n=\frac{c}{v}
$$

Where $v$ is the speed of the light in the medium and $c$ is the vacuum speed of the light.

Table 5.1 presents the refractive indices of some common liquids at a temperature of 20 degrees Celsius and wavelength of $589.29 \mathrm{~nm}$. The value of $n$ in a specific medium can depend on the wavelength of the light. The refractive index of a mixture also depends on its temperature and concentration. Thus, a liquid with a temperature profile has different refractive indices across the profile. The variation of the index of refraction with temperature is used in optical techniques in order to experimentally study heat and mass transfer in transparent liquids. In other words, the variation of the temperature and the concentration in a mixture establishes a spatial variation of the index of refraction and a corresponding phase shift in laser light passing through it. By means of optical interferometry and finding the phase shift, the temperature and concentration field can be found.

Table 5.1: Refractive index of some common liquids at $20^{\circ} \mathrm{C}$ and wavelength of $589.29 \mathrm{~nm}$

\begin{tabular}{cc}
\hline Material & Refractive index, $\boldsymbol{n}$ \\
\hline Benzene & 1.501 \\
Carbon disulfide & 1.628 \\
Carbon tetrachloride & 1.461 \\
Ethanol & 1.361 \\
Water & 1.3330 \\
\hline
\end{tabular}


To find the variation of the refractive index caused by the temperature and the concentration, for a given wavelength, the following equations are used:

\section{Binary systems:}

$$
\begin{aligned}
& \Delta n(x, z)=\left(\frac{\partial n}{\partial T}\right)_{T_{O}, C_{O}, \lambda} \Delta T(x, z)+\left(\frac{\partial n}{\partial c}\right)_{T_{O}, C_{O}, \lambda} \Delta c(x, z) \\
& T_{\text {glass }}=T_{0}+\Delta T[z / h-0.5] \\
& T=T_{0}+\left[\Delta \varphi-\Delta \varphi_{\text {ref } 1}-\Delta \varphi_{\text {glass }}\right] \times \frac{\lambda}{2 \pi L}\left(\frac{\partial n}{\partial T}\right)^{-1} \\
& c=c_{0}+\left[\Delta \varphi-\Delta \varphi_{\text {ref } 2}\right] \times \frac{\lambda}{2 \pi L}\left(\frac{\partial n}{\partial c}\right)^{-1}
\end{aligned}
$$

where $\Delta \varphi_{\text {ref } 1}$ phase change in the first reference image including the phase change in optical instruments. The second reference $\Delta \varphi_{\text {ref } 2}$ contains phase changes in thermal time and glass as well as $\Delta \varphi_{\text {ref } 1}$. These reference images are described in more details in section 5.3.1.

\section{Ternary systems:}

$$
\Delta n(x, z)=\left(\frac{\partial n}{\partial T}\right)_{C_{10}, C_{20}} \Delta T(x, z)+\left(\frac{\partial n}{\partial C_{1}}\right)_{T_{0}, C_{20}} \Delta C_{1}(x, z)+\left(\frac{\partial n}{\partial C_{2}}\right)_{T_{0}, C_{10}} \Delta C_{2}(x, z)
$$

$$
\left(\frac{\partial n}{\partial T}\right)_{T_{O}, C_{O}, \lambda} \text { and }\left(\frac{\partial n}{\partial C}\right)_{T_{O}, C_{O}, \lambda} \text { are the variation of the refractive index with the }
$$

temperature and the concentration, respectively, and named contrast factors. These values were obtained from [84] and [85].

$$
\begin{gathered}
\left(\frac{\partial n}{\partial c_{i}}\right)_{p, T, c_{j \neq i}=a_{0}}+a_{1} c_{2}+a_{2} c_{2}^{2}+a_{3} c_{2}^{3}+a_{4} c_{1}+a_{5} c_{1} c_{2}+a_{6} c_{1} c_{2}^{2} \\
+a_{7} c_{1}^{2}+a_{8} c_{1}^{2} c_{2}+a_{9} c_{1}^{3}
\end{gathered}
$$


Where $c_{i}$ indicates the mass fraction of the component $i\left(c_{1}+c_{2}+c_{3}=1\right)$. The values of $a_{k}$ for two wavelengths are presented in Table 5.2. The corresponding contrast factors are accurate for mixtures containing THN higher than $75 \%$. For mixtures with lower content of THN Equation (5.5) is not recommended and there is no other reference available in the literature.

Table 5.2: $a_{k}$ values for Equation (5.5)

\begin{tabular}{ccccccc} 
& & $\left(\frac{\partial n}{\partial c_{1}}\right)_{c_{2}}$ & & $\left(\frac{\partial n}{\partial c_{2}}\right)_{c_{1}}$ & & $\left(\frac{\partial n}{\partial c_{2}}\right)_{c_{3}}$ \\
\hline wavelength & $925 \mathrm{~nm}$ & $670 \mathrm{~nm}$ & $925 \mathrm{~nm}$ & $670 \mathrm{~nm}$ & $925 \mathrm{~nm}$ & $670 \mathrm{~nm}$ \\
$a_{0}$ & 0.085095 & 0.086586 & 0.045621 & 0.049960 & -0.058497 & -0.058599 \\
$a_{1}$ & 0.033512 & 0.040227 & 0.032737 & 0.046352 & 0.034343 & 0.046683 \\
$a_{2}$ & 0.000348 & -0.023488 & -0.018378 & -0.049073 & -0.018350 & -0.056080 \\
$a_{3}$ & 0.003391 & 0.025903 & 0.013930 & 0.034332 & 0.003030 & 0.028371 \\
$a_{4}$ & 0.040245 & 0.059863 & 0.033512 & 0.040227 & 0.019254 & 0.006907 \\
$a_{5}$ & -0.000571 & -0.018788 & 0.000696 & -0.046976 & -0.006807 & -0.048547 \\
$a_{6}$ & 0.023952 & 0.049013 & 0.010174 & 0.077709 & -0.006490 & 0.067400 \\
$a_{7}$ & 0.008975 & -0.038427 & -0.000285 & -0.009394 & -0.020961 & 0.010644 \\
$a_{8}$ & 0.039532 & 0.066725 & 0.023952 & 0.049013 & -0.008292 & 0.020922 \\
$a_{9}$ & 0.016207 & 0.050613 & 0.013177 & 0.022242 & 0.011767 & -0.009609 \\
\hline
\end{tabular}

\subsubsection{Subtraction of the reference image}

The phase pattern obtained from the fringes has information about several parameters. The parameters of interest are temperature ad concentration. Thus, the remained must be somehow subtracted. The following equation shows the parameters affecting the phase pattern. In each step of the experiment, one or more of them can be involved. 


$$
\Delta \varphi=\Delta \varphi_{\text {optics }}+\Delta \varphi_{\text {glass }}+\Delta \varphi_{t h}+\Delta \varphi_{C}
$$

To explain the subtraction process, a typical example of binary experiments is described here.

After installing the cell on the bench in the Mach-Zehnder setup, the mean temperature is applied to the top and bottom sides of the cell. The first image is taken after 20 minutes. At this time, it is assumed that the whole mixture is at $25{ }^{\circ} \mathrm{C}$. This fringe pattern is the first reference image. The corresponding phase pattern includes information about the phase change due to the optical instruments. As the beam passes through the optical elements, it is distorted and phase change happens.

$$
\Delta \varphi=\Delta \varphi_{R e f 1}=\Delta \varphi_{\text {optics }}
$$

After a few minutes, the temperature gradient is applied to the cell. The time takes that a steady temperature profile established is named the thermal characteristic time, $\tau_{t h}$ :

$$
\tau_{t h}=h^{2} / \chi
$$

where $h$ is the height of the cell, and $\chi$ is the thermal diffusivity of the mixture. A thermal characteristic time is approximately 3-4 minutes. The phase pattern during this period of the experiment has the information about the temperature in the mixture as well as temperature in the liquid. However, due to the longer diffusion characteristic time, $\tau_{D}$, the contribution of the concentration to the phase change is negligible.

$$
\tau_{D}=h^{2} / D
$$


where $D$ is the diffusion coefficient. For the benchmark mixtures, thermal time is $3-4$ minutes while the diffusion time is about 12 hours. Therefore, separation of the components occurs much slower than the formation of a steady temperature profile.

During the thermal time, as the concentration contribution to the phase change is ignored, the refractive index variation is due only to the temperature variation and Equation (5.2) is simplified to:

$$
\Delta n(x, z)=\left(\frac{\partial n}{\partial T}\right)_{T_{O}, C_{o}, \lambda} \Delta T(x, z)
$$

The temperature profile in the glass is linear:

$$
T_{\text {glass }}=T_{0}+\Delta T[z / h-0.5]
$$

The contribution of the changes in $T_{\text {glass }}$ to the phase change is:

$$
\Delta \varphi_{\text {glass }}=\left[T_{0}+\Delta T(z / h-0.5)\right] \times\left(\frac{\partial n}{\partial T}\right)_{\text {glass }} \frac{2 \pi L_{\text {glass }}}{\lambda}
$$

The temperature profile in the mixture is found as follows:

$$
T=T_{0}+\left[\Delta \varphi-\Delta \varphi_{\text {ref } 1}-\Delta \varphi_{\text {glass }}\right] \times \frac{\lambda}{2 \pi L}\left(\frac{\partial n}{\partial T}\right)^{-1}
$$

From this point on, the temperature has no more contribution to the phase variation. A second reference image is taken at the end of the thermal characteristic time.

$$
\Delta \varphi_{\text {ref } 2}=\Delta \varphi_{R e f 1}+\Delta \varphi_{\text {glass }}+\Delta \varphi_{t h}
$$


The last equation has the information of all factors that affect the phase except the concentration. Thus, any phase change that occurs after this point in time, is caused only by the concentration gradient.

$$
c=c_{0}+\left[\Delta \varphi-\Delta \varphi_{r e f 2}\right] \times \frac{\lambda}{2 \pi L}\left(\frac{\partial n}{\partial c}\right)^{-1}
$$

\subsection{ERROR ANALYSIS}

The main goal of this section is to find the uncertainty in the measurement of the Soret coefficients, concentration and temperature distributions of both binary and ternary systems. The parameters and their associated uncertainties are listed in Table 5.3.

Table 5.3: Measured values and their estimated uncertainties

\begin{tabular}{cccc}
\hline Parameter & Symbol & Measured value & $\begin{array}{c}\text { Absolute } \\
\text { uncertainty }\left(\delta x_{i}\right)\end{array}$ \\
\hline $\begin{array}{c}\text { Temperature (using } \\
\text { thermistor) }\end{array}$ & $\mathrm{T}$ & 25 Celsius & 0.01 \\
Concentration & $\mathrm{c}$ & 2.50 grams & 0.0002 \\
Height of cell & $\mathrm{H}$ & 2.5 and $5 \mathrm{~mm}$ & $\pm 5 \times 10^{-5}$ \\
Length of cell & $\mathrm{L}$ & 10 and $15 \mathrm{~mm}$ & $\pm 5 \times 10^{-5}$ \\
Wavelength & $\lambda$ & 632.8 and $940 \mathrm{~nm}$ & $\pm 0.1 \mathrm{~nm}$ \\
\hline
\end{tabular}

If $R$ is a calculated value obtained from experiments, and $x_{1}, x_{2}, \ldots, x_{n}$ are the independent parameters, by using the root-sum-square method, the uncertainty of $R$ can be found: 


$$
\delta R=\sqrt{\left(\frac{\delta R}{\delta x_{1}} \delta x_{1}\right)^{2}+\left(\frac{\delta R}{\delta x_{2}} \delta x_{2}\right)^{2}+\ldots+\left(\frac{\delta R}{\delta x_{n}} \delta x_{n}\right)^{2}}
$$

where $\delta x_{1}, \delta x_{2}, \ldots, \delta x_{n}$ are the random uncertainties.

\subsubsection{Uncertainty of temperature}

The temperature along the vertical cross section in the centre of the cell is calculated as follows:

$$
\begin{gathered}
T=T_{0}+\Delta T \\
\Delta T=\frac{\Delta n}{(\partial n / \partial T)_{\lambda, T_{O}, C_{O}}}
\end{gathered}
$$

The related uncertainty is calculated as follows:

$$
\delta(T)=\delta\left(T_{0}\right)+\delta(\Delta T)
$$

where $\delta\left(T_{0}\right)$ is the uncertainty of the mean temperature measured by the thermistor. It is known to be $0.01{ }^{\circ} \mathrm{C}$. By applying the RSS method to Equation (5.18), the uncertainty of $\Delta T$ is found: 


$$
\delta(\Delta T)=\sqrt{\left(\frac{\delta(\Delta n)}{(\partial n / \partial T)_{\lambda, T_{o}, C_{o}}}\right)^{2}+\left(\frac{\Delta n \delta(\partial n / \partial T)}{(\partial n / \partial T)^{2}}\right)^{2}}
$$

$\Delta n$ is obtained from interferometry and the calculated phase change:

$$
\Delta n=\frac{\lambda \Delta \varphi}{2 \pi L}
$$

The related uncertainty is calculated as follows:

$$
\delta(\Delta n)=\sqrt{\left(\frac{\lambda}{2 \pi L} \delta(\Delta \varphi)\right)^{2}+\left(\frac{\Delta \varphi \cdot \lambda}{2 \pi}\left(\frac{-1}{L^{2}}\right) \delta(\Delta L)\right)^{2}+\left(\frac{\Delta \varphi}{2 \pi L} \delta(\lambda)\right)^{2}}
$$

As seen in Equation (5.22), the uncertainty of $\Delta n$ is a function of the phase change, which varies during the experiment.

Figure 5.3 illustrates the temperature profile on a vertical line at center of the cell with the corresponding error bars. 


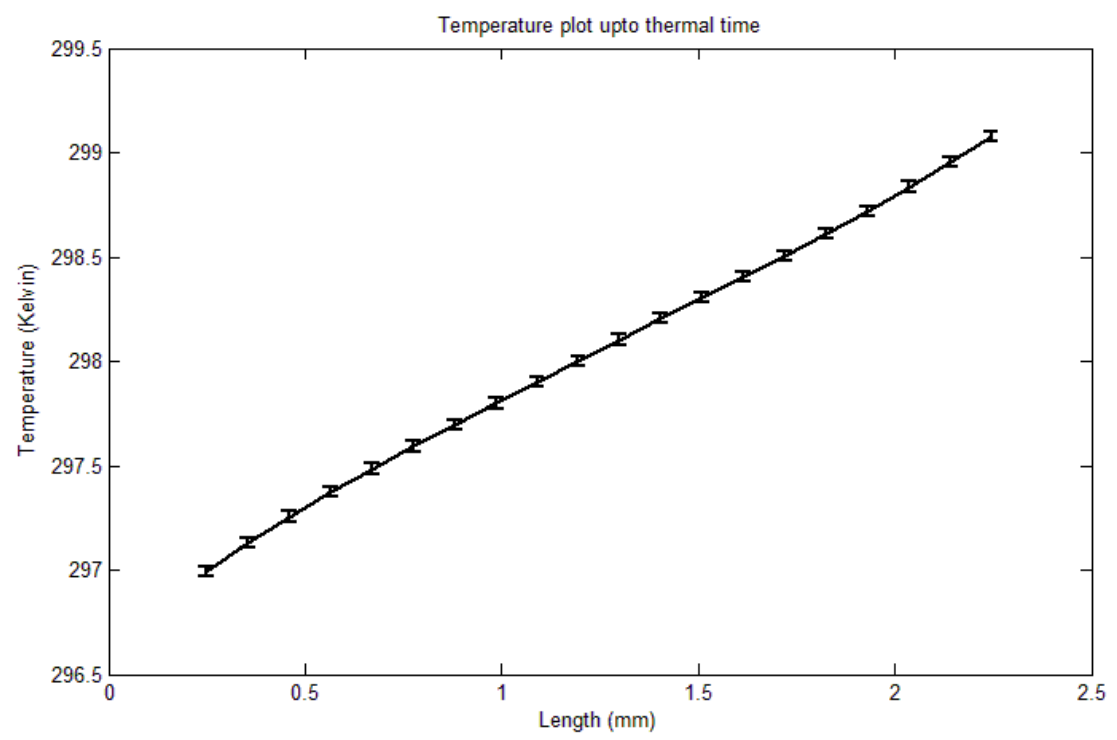

Figure 5.3: Temperature distribution along the vertical line in the centre of the cell with error bars

\subsubsection{Uncertainty of concentration}

The concentration along the vertical in centre of the cell is calculated as follows:

$$
\begin{gathered}
c=c_{0}+\Delta c \\
\Delta c=\frac{\Delta n}{(\partial n / \partial c)_{\lambda, T_{o}, c_{O}}}
\end{gathered}
$$

The related uncertainty is calculated as follows:

$$
\delta(c)=\delta\left(c_{0}\right)+\delta(\Delta c)
$$


$\delta\left(C_{0}\right)$ is the uncertainty of the mean concentration as measured by the weight measurement on the weight scale, which is $2 \mathrm{e}-4$.

By applying the RSS method to Equation (5.18) the uncertainty of $\Delta T$ is found:

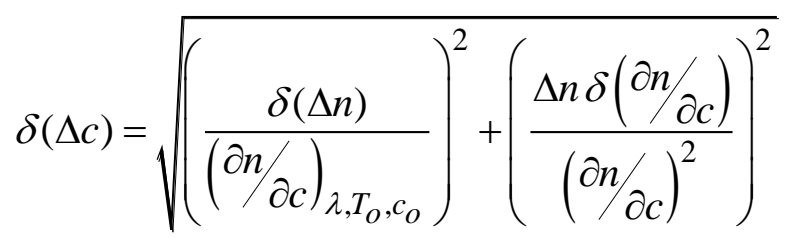

\subsubsection{Uncertainty of the Soret coefficient}

By applying the root sum square (RSS) method to the equation for the Soret coefficient, the uncertainty of the Soret coefficient measurement is obtained:

$$
S_{T}=\frac{-1}{C_{0}\left(1-C_{0}\right)} \frac{\Delta c}{\Delta T}
$$

For the binary mixtures that are being used in this study, the mass fraction is 0.50 . Therefore,

$$
\begin{gathered}
c_{0}\left(1-c_{0}\right)=c_{0}^{2} \\
\delta\left(S_{T}\right)=\operatorname{Sqrt}\left[\left(\frac{-1}{c_{0}{ }^{2}} \cdot \frac{1}{\Delta T} \delta(\Delta c)\right)^{2}+\left(\frac{-\Delta c}{c_{0}{ }^{2}} \cdot \frac{-1}{\Delta T^{2}} \delta(\Delta T)\right)^{2}+\left(\frac{-\Delta c}{\Delta T} \cdot \frac{-2}{c_{0}^{3}} \delta\left(\Delta c_{0}\right)\right)^{2}\right]
\end{gathered}
$$

Figure 5.4 depicts the evolution of the maximum concentration difference between the hot and cold sides of the cell with the corresponding error bars. 


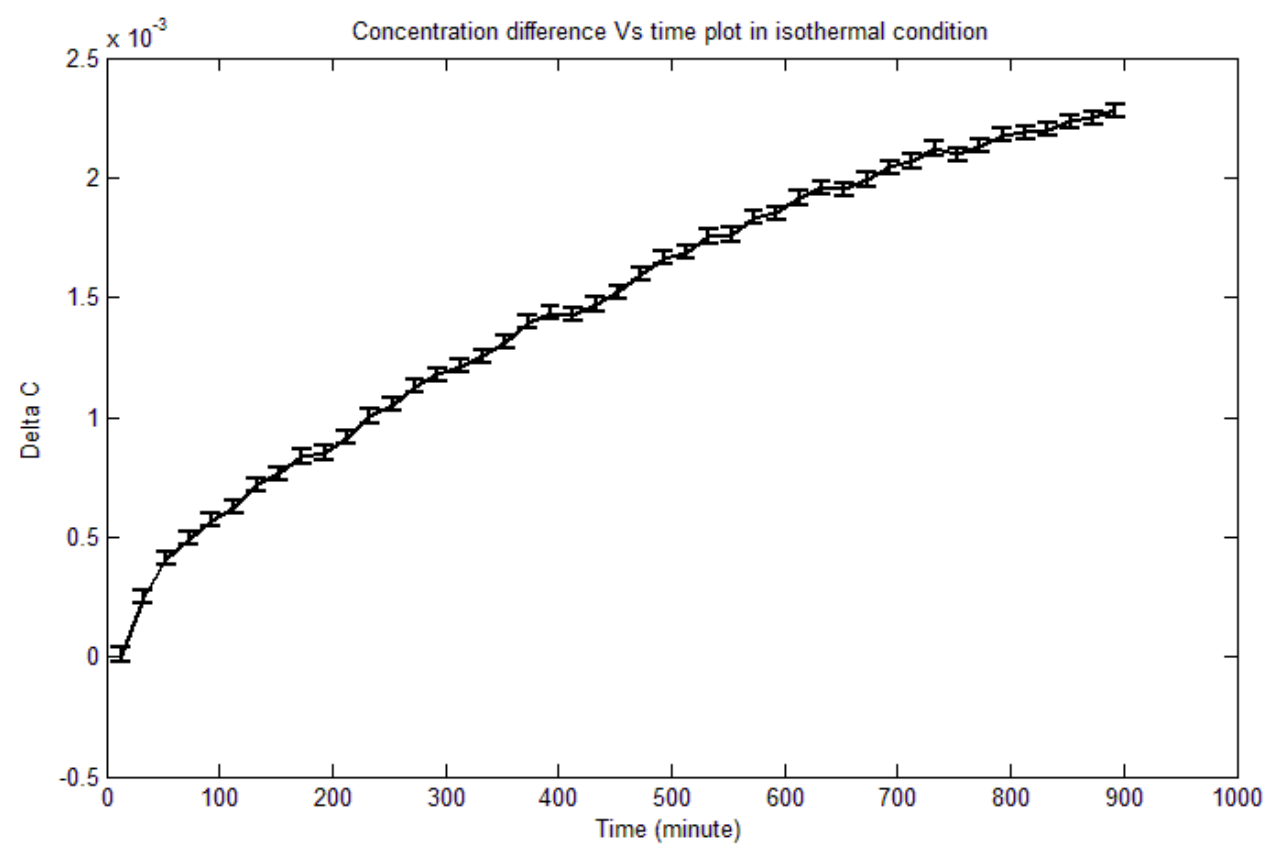

Figure 5.4: The evolution of the concentration difference between the hot and cold sides with error bars

\subsubsection{Uncertainty in the concentration difference in ternary mixtures}

As discussed earlier, in ternary systems, the changes of the refractive index obtained from two different wavelengths are defined as follows:

$$
\begin{gathered}
\Delta n_{1}=\left(\frac{\partial n_{1}}{\partial c_{1}}\right)_{\lambda_{1}, T_{0}, c_{2}} \Delta c_{1}+\left(\frac{\partial n_{1}}{\partial c_{2}}\right)_{\lambda_{1}, T_{0}, c_{1}} \Delta c_{2} \\
\Delta n_{2}=\left(\frac{\partial n_{2}}{\partial c_{1}}\right)_{\lambda_{2}, T_{0}, c_{2}} \Delta c_{1}+\left(\frac{\partial n_{2}}{\partial c_{2}}\right)_{\lambda_{2}, T_{0}, c_{1}} \Delta c_{2}
\end{gathered}
$$

Thus, the changes of the concentration of the two components are calculated as

$$
\Delta C_{1}=\frac{B_{2} E_{1}-B_{1} E_{2}}{B_{2} A_{1}-B_{1} A_{2}}
$$




$$
\Delta C_{2}=\frac{A_{1} E_{2}-A_{2} E_{1}}{B_{2} A_{1}-B_{1} A_{2}}
$$

$$
\begin{array}{cc}
A_{1}=\left(\frac{\partial n_{1}}{\partial C_{1}}\right)_{\lambda_{1}, T_{0}, C_{2}}, & A_{2}=\left(\frac{\partial n_{2}}{\partial C_{1}}\right)_{\lambda_{2}, T_{0}, C_{2}} \\
B_{1}=\left(\frac{\partial n_{1}}{\partial C_{2}}\right)_{\lambda_{1}, T_{0}, C_{1}}, & B_{2}=\left(\frac{\partial n_{2}}{\partial C_{2}}\right)_{\lambda_{2}, T_{0}, C_{1}} \\
E_{1}=\Delta n_{1}, & E_{2}=\Delta n_{2}
\end{array}
$$

By applying the RSS method to Equation (5.25), the uncertainty of $\Delta C_{1}$ is obtained. The same expression can be used to find $\Delta C_{2}$.

$$
\begin{array}{ll}
\delta\left(\Delta C_{1}\right)=\sqrt{\left(\frac{\delta f}{g}\right)^{2}+\left(\frac{f \delta g}{g^{2}}\right)^{2}} & \\
f=B_{2} E_{1}-B_{1} E_{2}, & g=B_{2} A_{1}-B_{1} A_{2}
\end{array}
$$

The calculated parameters and the related uncertainties are tabulated in Table 5.4.

Table 5.4: Calculated uncertainties

\begin{tabular}{cc}
\hline$\delta(\Delta n)$ & $\pm 3.18 \times 10^{-6}$ \\
$\delta(\Delta T)$ & \pm 0.0238 \\
$\delta(\Delta C)$ & $\pm 5 \times 10^{-5}$ \\
$\delta\left(S_{T}\right)$ & $\pm 8.3 \times 10^{-5}$ \\
\hline
\end{tabular}




\subsection{CONCLUSIONS}

By image processing the captured fringe patterns from interferometry, the variations of the refractive index were obtained. Thereafter, the concentration and temperature fields were found from the refractive index changes. 2-D FFT method was used to extract the information from the fringe patterns. Afterwards, the uncertainty of experimental measurement was analysed by using root-sum-square method. The accuracy of the measured temperature, concentration and Soret coefficient for binary and ternary mixtures were calculated and the error bars were shown on temperature and concentration profiles. 


\section{MEASURED SORET COEFFICIENT FOR TERNARY MIXTURES}

\subsection{INTRODUCTION}

Binary and ternary hydrocarbon mixtures were used to obtain the corresponding concentration profiles and Soret coefficients. The thermal design of the cell was studied earlier in chapter 4 . The effect of the inclination was also presented.

The binary mixture of $\mathrm{THN}-\mathrm{C}_{12}$ with a mass fraction of $50 / 50$ was used to validate the experimental setup and the measurement technique. This mixture - has been well-studied, and the Soret coefficient is available as a Fontainebleau benchmark.

The Soret effect studied in five ternary hydrocarbon mixtures composed of THN-IBB-C 12 with mass fractions of: $80 / 10 / 10,70 / 10 / 20,73.4 / 10.3 / 16.3,71.8 / 20.2 / 8,81.9 / 10 / 8.1$. The Soret coefficient and concentration profile were measured. The first mass fraction has been already studied by other research groups and the measured Soret coefficients are available in the literature. The reason of testing this mixture again, was to validate the experiment setup used in the current study. This is the first detailed report on the other four the compositions using ground based optical interferometry.

\subsection{ChAPTER AIMS}

In the current chapter, experimental results for the ternary systems are presented. Using the results for the binary mixture, it is shown that the experimental measurements were validated by comparing to the published benchmark values. Finally, the results from the ternary system experiments are presented and discussed. The proposed values of $S_{T}$ are tabulated.

\subsection{VALIDATION}

The preliminary tests were done on the binary mixture of $\mathrm{THN}-\mathrm{C}_{12}$ with a mass fraction of $50 / 50$ at a mean temperature of $25^{\circ} \mathrm{C}$. The obtained fringe patterns are shown in Figure 6.1. During the first few minutes of the experiment, when the sides were still at the same temperature and the temperature gradient had not yet been applied, the fringes were thick. As soon as the temperature 
gradient was applied, the fringes became thinner. The thickness of the fringes was a function of the refractive index, which varied with temperature and concentration.

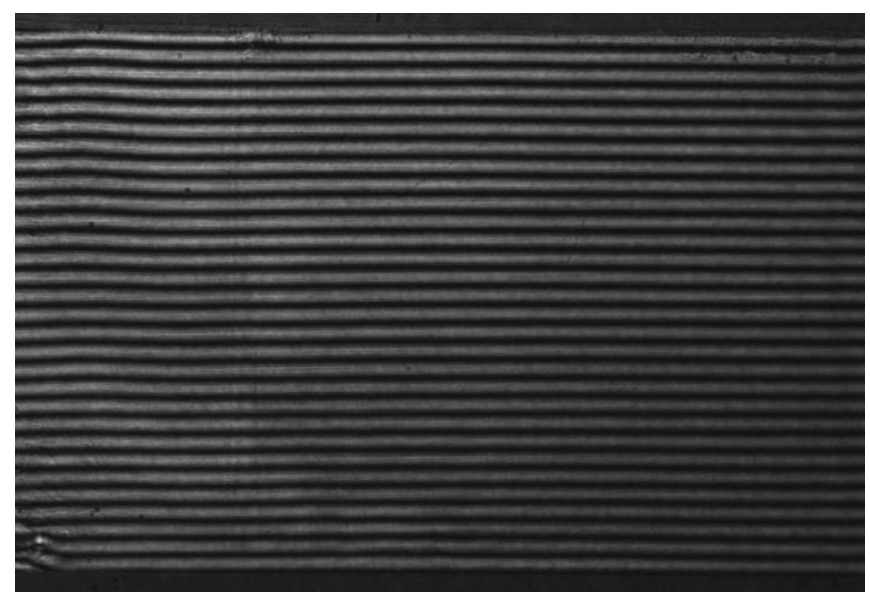

(a)

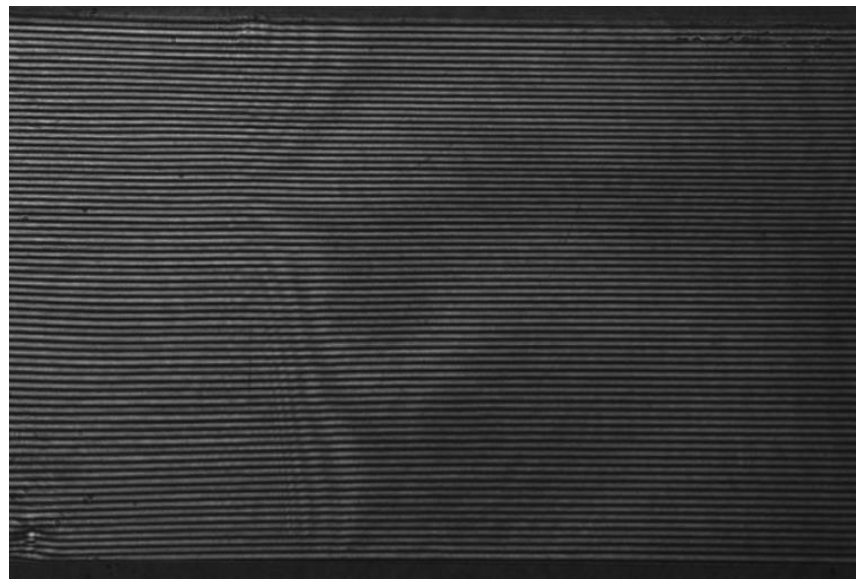

(b)

Figure 6.1: Fringe pattern of a binary mixture under (a) isothermal conditions (b) with a temperature gradient

By using 2D-FFT method, the phase distribution of the fringe patterns was extracted. The unwrapped phase band of a randomly chosen fringe pattern of the cell with a temperature gradient is shown in Figure 6.2. The phase value in each pixel was used to find the temperature and concentration in that pixel. 


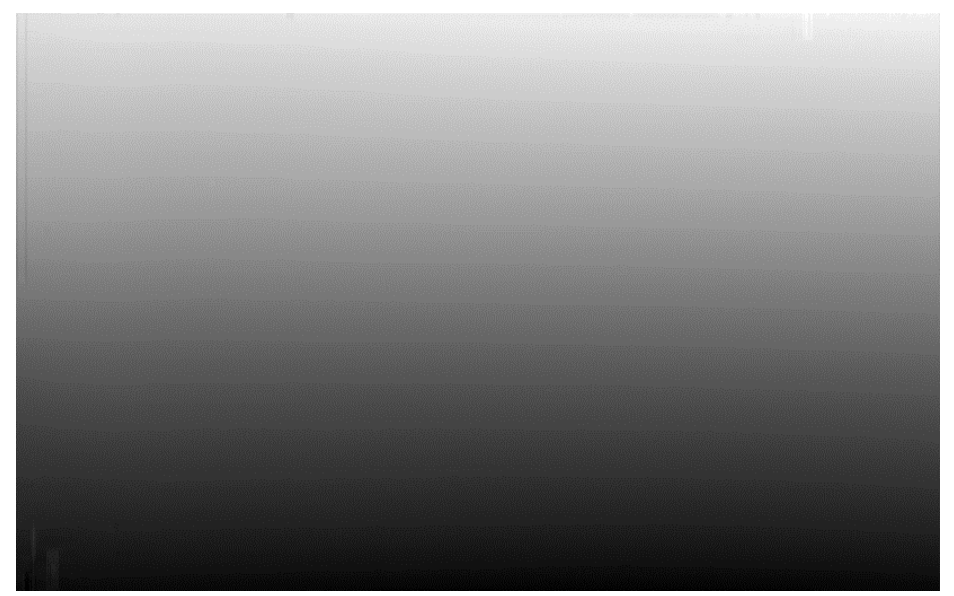

\section{Figure 6.2: Unwrapped phase band including information about temperature and concentration profiles}

As discussed earlier in chapter 5, the contribution of the concentration variation to the refractive index during the thermal time is negligible. This phenomenon can be observed in the evolution of the refractive index as shown in Figure 6.3. Each point on the trend line represents the difference between the refractive indices at the hot and cold sides at a certain time $\left(\Delta n=n_{\text {hot }}-n_{\text {cold }}\right)$. There was a sudden increase in $\Delta n$ at time $=4 \mathrm{~min}$. This sharp jump occurred right after application of the temperature gradient. After establishing a steady temperature gradient in the mixture after 3 minutes, $\Delta n$ increased gradually and slowly through the next 200 minutes due to the separation of the components. By using this information, the refractive index values of the first 7 images were used to find the temperature profile from Equation 5.10. The CCD camera took a picture every minute. Therefore, picture number 7 represents the seventh minute from the zero time.

The temperature distribution in the cell at different times is shown in Figure 6.4. The first image shows the stationary temperature field in the cell. The next image shows the temperature distribution after the temperature gradient was established. The unclear distribution of the temperature near the hot side and left lower corner were due to defects in the image processing. 


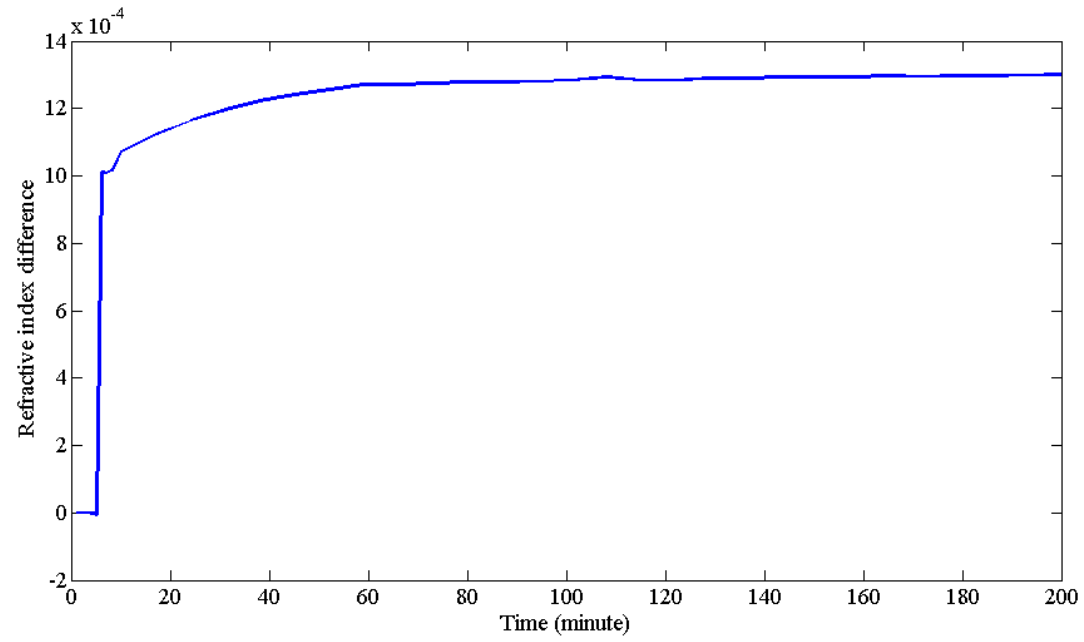

Figure 6.3: Evolution of the refractive index vs. time

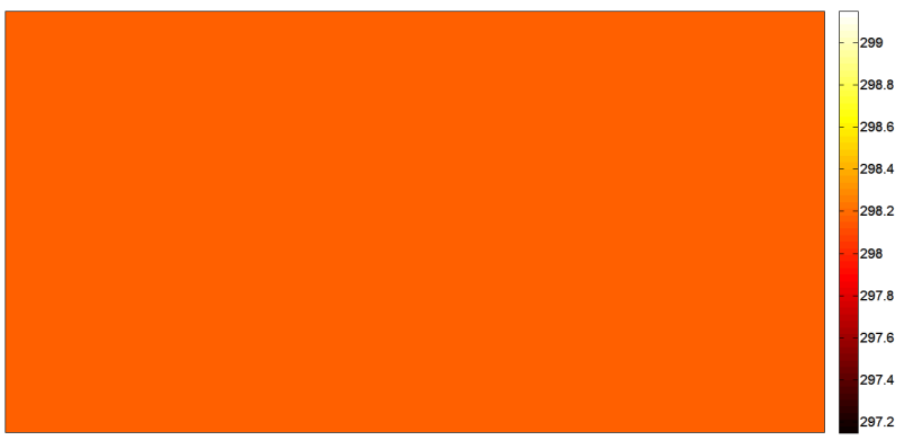

(a)

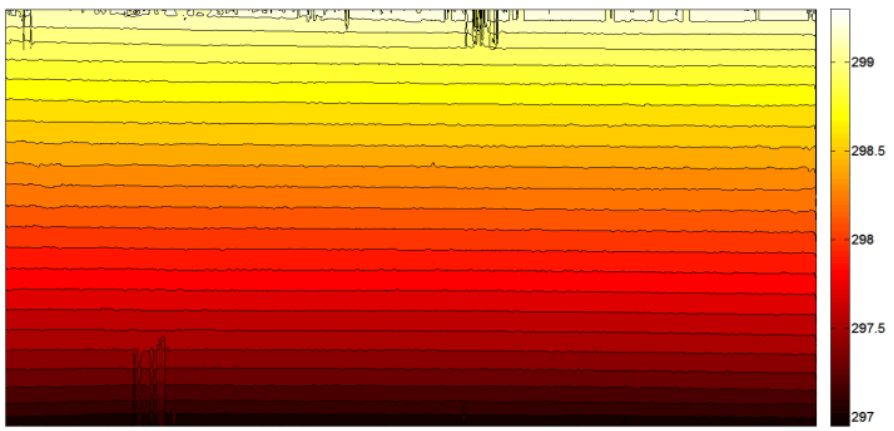

(b)

Figure 6.4: Temperature distribution obtained experimentally at (a) $t=0$ and (b) $t=7$ minutes 
Figure 6.5 depicts the temperature profile on a vertical line at the centre of the cell from bottom to top. The profile was approximately linear, which describes an almost purely conductive heat transfer in the mixture with minimal convection motions.

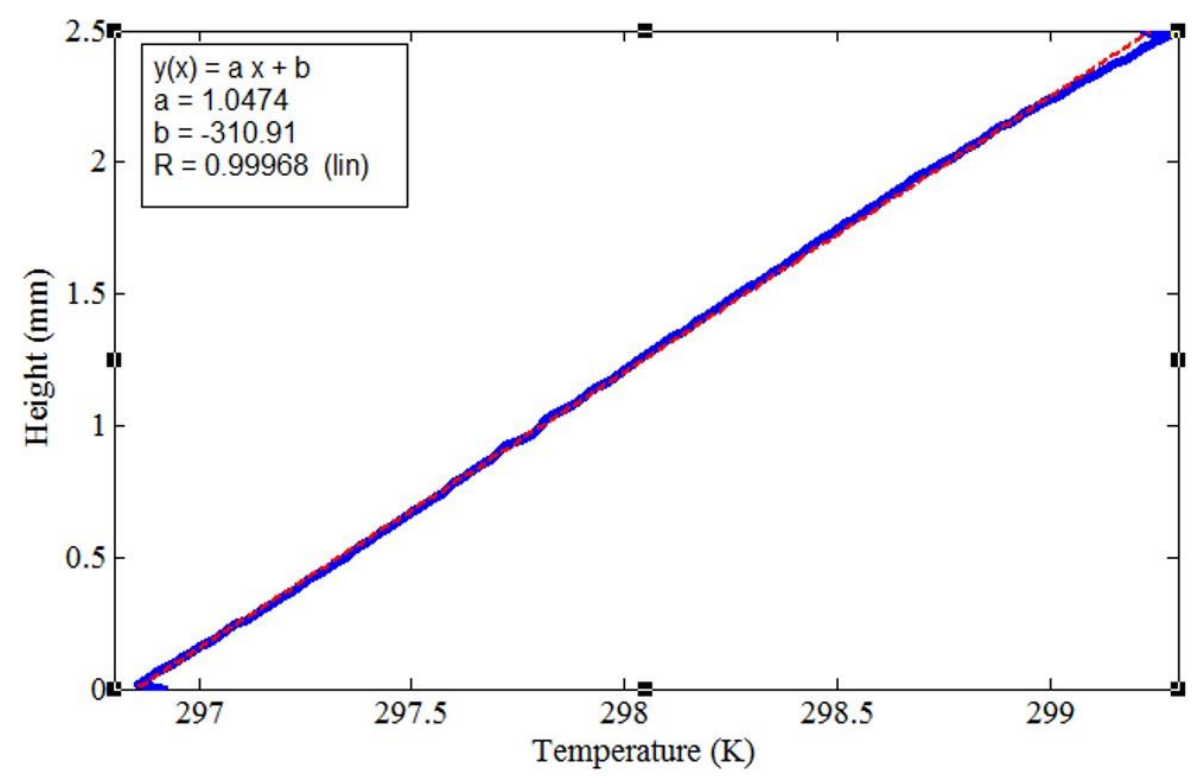

Figure 6.5: Temperature profile from the cold side to the hot side

The concentration fields at different times during the separation time are illustrated in Figure 6.6. Figure 6.6(a) shows a uniform field of the concentration distribution in the cavity before application of the temperature gradient. The uniform distribution of the components at this time shows that the separation had not commenced. Figure 6.6(b) depicts the concentration field after 150 minutes. The temperature field was established after approximately 14 minutes of the start point of the experiment. After 150 minutes, the components have been separated, but the separation had not yet reached a steady state. After 200 minutes, the system reached the steady state, which is shown in Figure 6.6(c).

Figure 6.7 shows the concentration variation along a vertical line at the centre of the cell from bottom to top at different time steps. The values were averaged along the horizontal direction. The yellow and the black lines represent the concentration profiles after 150 and 200 minutes, respectively. The regions near the hot and cold sides were experiencing a non-linear concentration profile and were cropped out. Only the linear parts were used to calculate the Soret coefficients. 
The non-linearity of the concentration profile was due the convection residuals near the hot and cold sides.

Figure 6.8 shows the evolution of $\Delta C$ of the binary benchmark mixture of THN-C $\mathrm{C}_{12}$ with a mass fraction of $50 / 50$ at the average temperature of $25{ }^{\circ} \mathrm{C}$. The maximum separation was $2.5 \times 10^{-3}$.

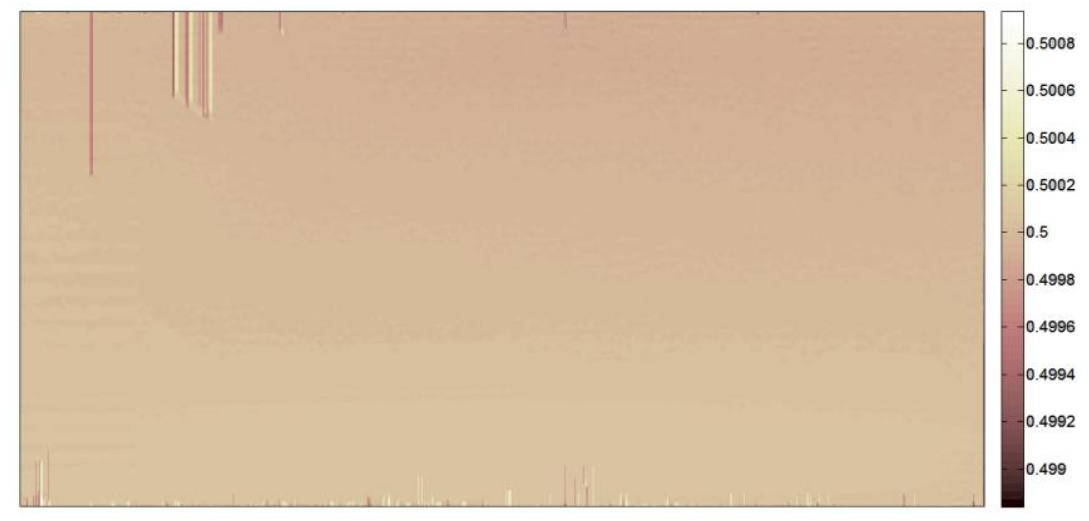

(a) $10 \mathrm{~min}$

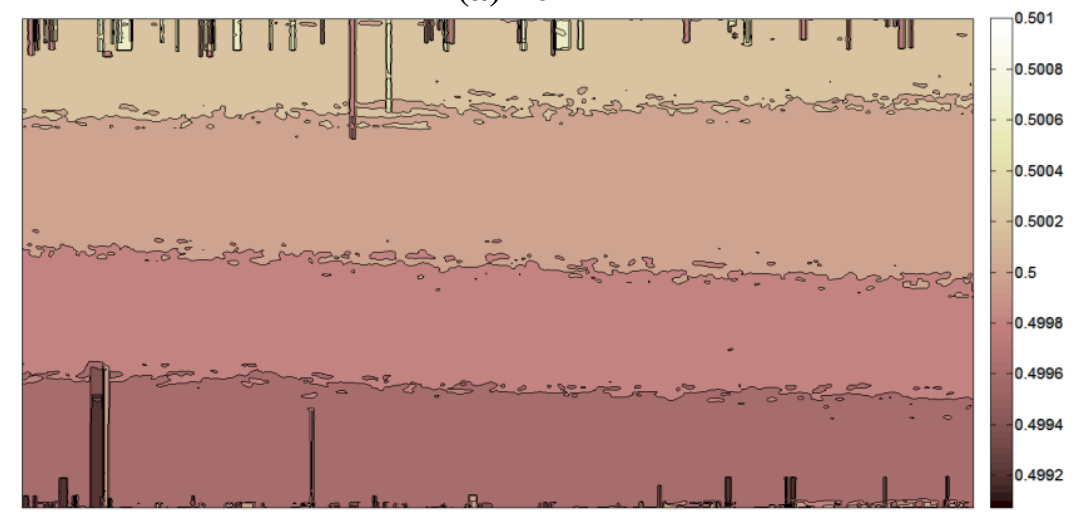

(b) $150 \mathrm{~min}$

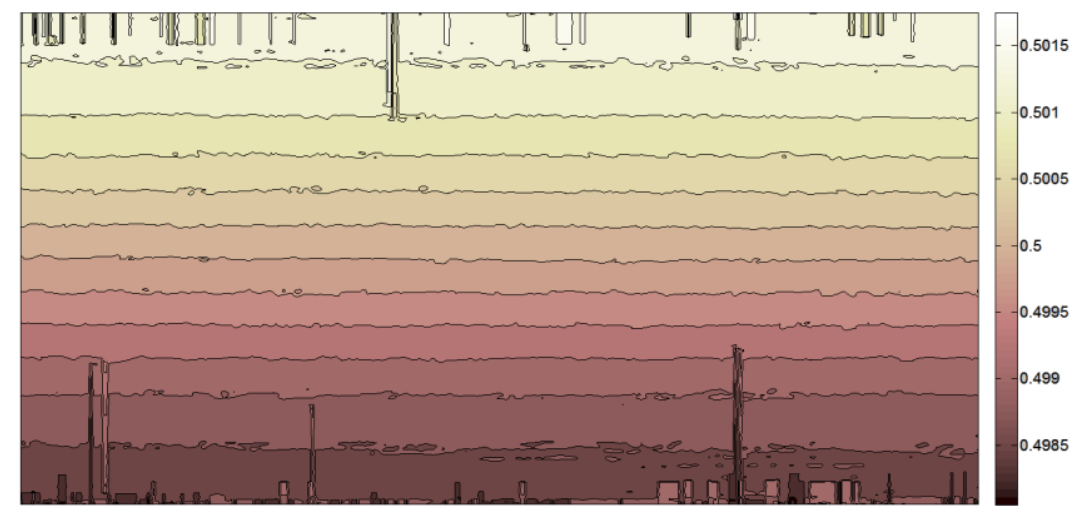

(c) $200 \mathrm{~min}$

Figure 6.6: Concentration contour at different times 


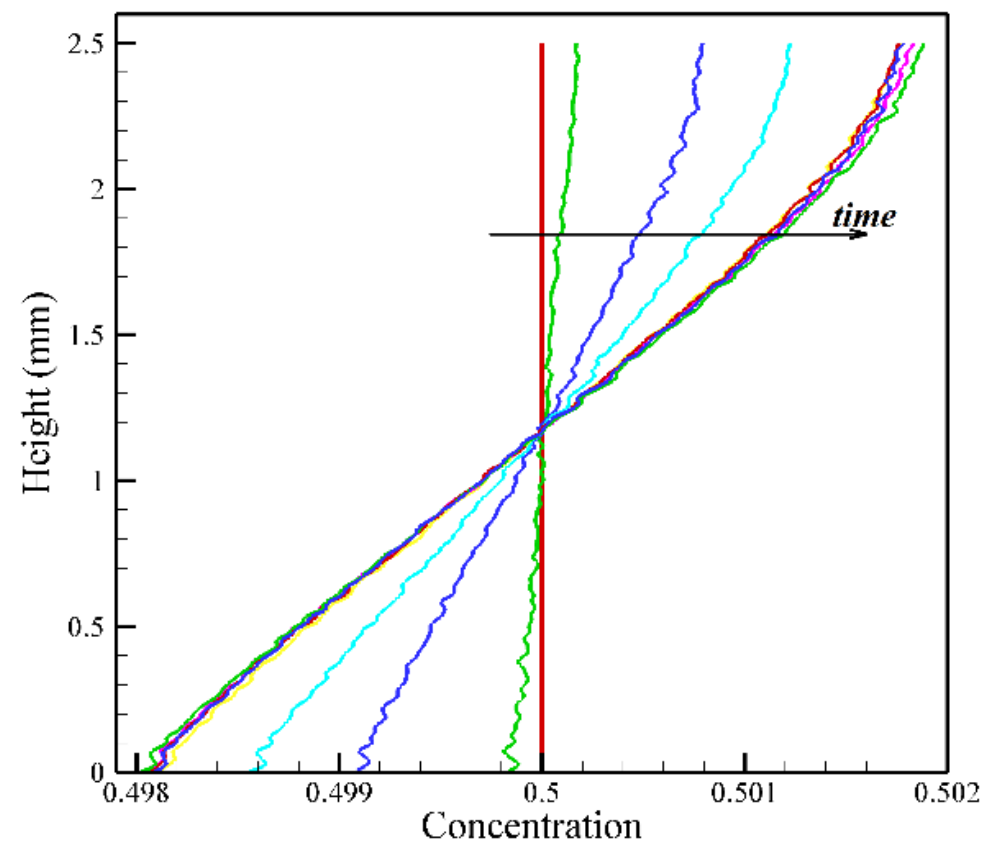

Figure 6.7: Concentration profile at different times

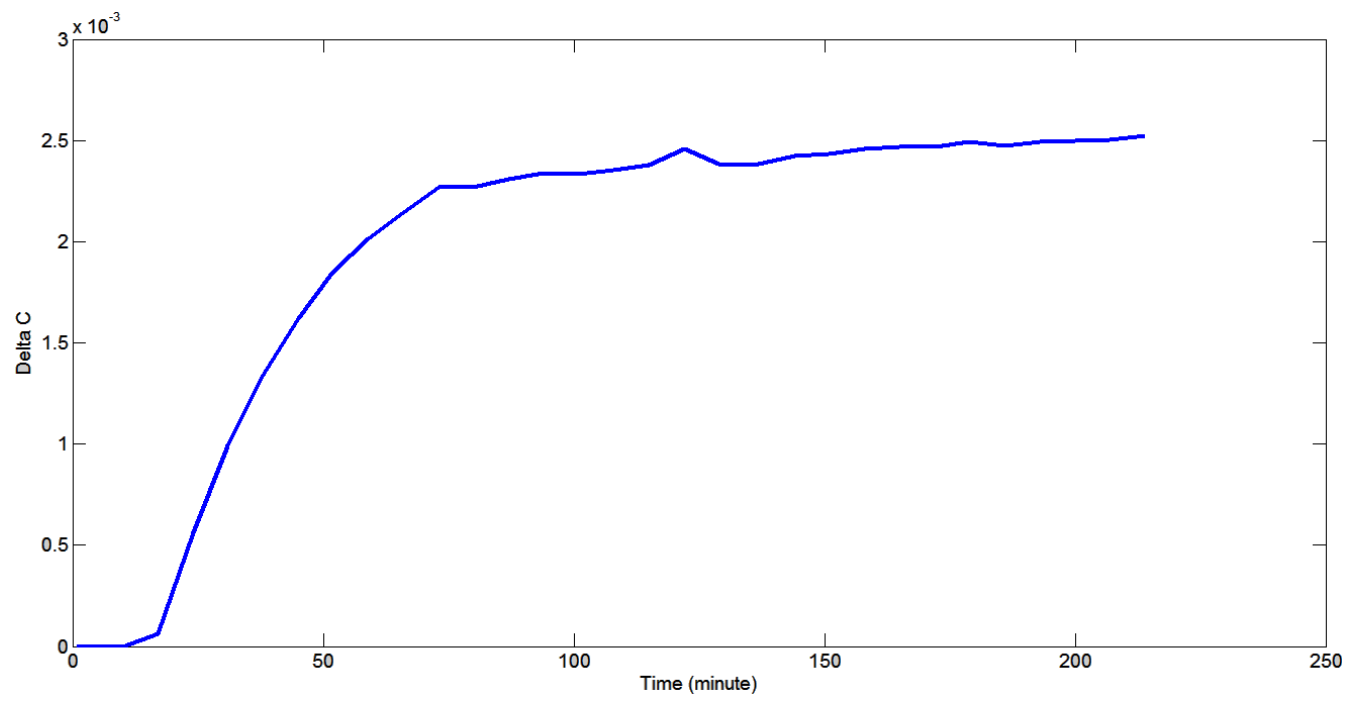

Figure 6.8: Evolution of $\Delta C$ of the $\mathrm{THN}-\mathrm{C}_{12}$ mixture

The measurement technique was validated by comparing to the results presented in [65], which have been validated by the Fontainebleau benchmark. However, the height of the cell in 
the present study was shorter. Therefore, the time needed to reach steady state was also shorter. The thermal diffusion time was estimated as

$$
\tau=\frac{h^{2}}{\pi^{2} D}
$$

The height of the cell in the present study was almost half of the one used in [65]. Therefore, it was expected that we would achieve the maximum separation of components in one fourth of the time needed than if the cell had a height of $5 \mathrm{~mm}$. Figure 6.9 compares the evolution of $\Delta C$ obtained in [65] and in the present study. The thermal diffusion time for the cell with height of 5 $\mathrm{mm}$ is about 800 minutes. As expected, the thermal time was shorter in the present study, at about 200 minutes. However, the maximum separation in the current cell was smaller due to the smaller temperature gradient. The Soret coefficients obtained in this study and those from the literature are shown in Figure 6.9. A good agreement with the literature was obtained.

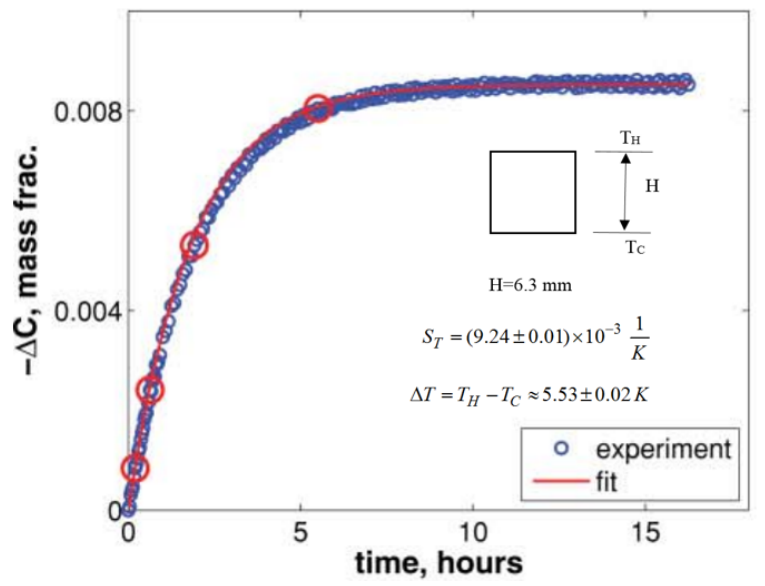

(a)

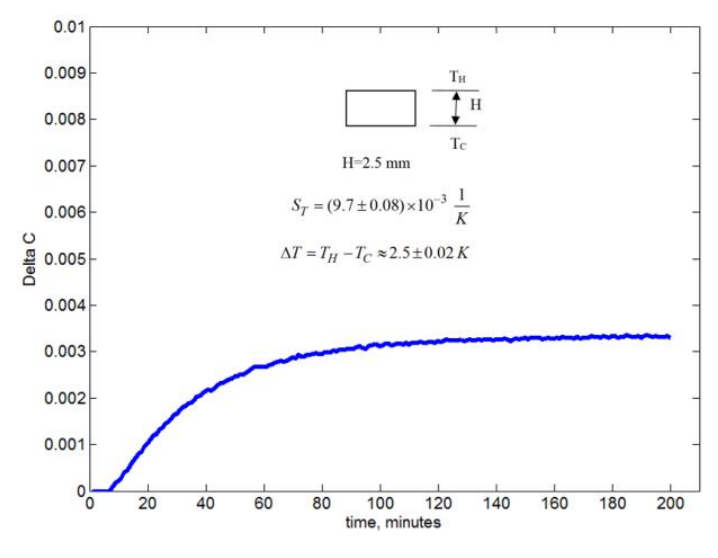

(b)

Figure 6.9: Evolution of the concentration difference between hot and cold walls (a) result of [65], (b) result of the current study 


\subsection{TERNARY SYSTEMS}

The experimental procedure for the ternary mixture was almost same as the binary experiments, including the isothermal and thermal diffusion steps.

The distribution of the concentration is calculated after the steady temperature gradient is established. For all the mixtures in the present study, the separation continued for almost 250 minutes. The measured concentration differences between the hot and cold walls $\left(C_{\text {hot }}-C_{\text {cold }}\right)$ at the maximum separation point are presented in Table 6.1. The separation of THN towards the cold side indicates a positive Soret effect while the positive concentration difference for the other components shows a negative effect. Ahadi et al. [72] noticed a same behaviour for the components in the mixture with mass fraction of 10/80/10. The corresponding temperature gradients presented in Table 6.1 are not equal for different mixtures. The reason of the non-equal temperature gradients is the different cropped area for each case. The region near hot and cold walls affected by the beam deflection problem is different for each case. Thus the cropped area has not the same size for all cases. Consequently, the temperature gradient between the boundaries of a smaller cropped region is smaller than the total applied temperature difference.

Table 6.1: Maximum separation and corresponding temperature gradient for different compositions

\begin{tabular}{cccccc}
\hline Mixture\# & 1 & 2 & 3 & 4 & 5 \\
\hline$\Delta C_{\max }^{T H N} \times 10^{-3}$ & -1.98 & -4.148 & -3.80 & -2.51 & -2.68 \\
$\Delta C_{\max }^{I B B} \times 10^{-3}$ & 0.64 & 2.75 & 2.4 & 1.66 & 1.77 \\
$\Delta T(K)$ & 1.59 & 1.60 & 1.23 & 1.60 & 2.17 \\
\hline
\end{tabular}

\subsection{DETERMINATION OF SORET COEFFICIENT, $S_{T}$}

The Soret coefficient of the mixture with composition of $0.80 / 0.1 / 0.1$ was previously studied by other researchers [86-91] and this mixture was investigated again in the current study in order to validate the experiments. However, this is the first report on the Soret coefficient of the other four mixtures. The Soret coefficient for each component is calculated as follows: 


$$
S_{T}=-\frac{\Delta C_{\max }}{\Delta T}
$$

The repeatability of the experiments was checked by repeating the test for each mixture three times. The mean value of the Soret coefficient for the $0.80 / 0.10 / 0.10$ mixture and the benchmark values are presented in Table 6.2. The good agreement between the results of the current study and the benchmark values proves the validity of the experiments.

The proposed mean values of the Soret coefficient for the other mixtures are presented in Table 6.3. In all cases THN has a positive Soret coefficient while the other two components have negative values of $S_{T}$. The concentration difference of the third component $\left(\mathrm{C}_{12}\right)$ is obtained from $\Delta C_{T H N}+\Delta C_{I B B}+\Delta C_{C_{12}}=0$ and then by using Equation (6.2), $S_{T}^{C_{12}}$ is calculated.

Table 6.2: Measured and benchmark values of Soret coefficient for $0.80 / 0.10 / 0.10$ mixture

\begin{tabular}{cccccc}
\hline \multicolumn{3}{c}{ Measured values of $S_{T} \times 10^{-3}\left[K^{-1}\right]$} & \multicolumn{3}{c}{ Benchmark values of $S_{T} \times 10^{-3}\left[K^{-1}\right][89]$} \\
\hline THN & IBB & C12 & THN & IBB & $\mathrm{C}_{12}$ \\
\hline $1.24 \pm 0.090$ & $-0.40 \pm 0.075$ & $-0.84 \pm 0.083$ & $1.40 \pm 0.16$ & $-0.57 \pm 0.26$ & $-0.83 \pm 0.10$ \\
\hline
\end{tabular}

Table 6.3: Measured Soret coefficient for the new mixtures

\begin{tabular}{cccc}
\hline mixture number & $S_{T}^{T H N} \times 10^{-3}\left[K^{-1}\right]$ & $S_{T}^{I B B} \times 10^{-3}\left[K^{-1}\right]$ & $S_{T}^{C_{12}} \times 10^{-3}\left[K^{-1}\right]$ \\
\hline 2 & $2.66 \pm 0.051$ & $-1.76 \pm 0.060$ & $-0.90 \pm 0.055$ \\
3 & $2.92 \pm 0.095$ & $-1.87 \pm 0.080$ & $-1.05 \pm 0.86$ \\
4 & $1.52 \pm 0.079$ & $-1.0 \pm 0.048$ & $-0.52 \pm 0.065$ \\
5 & $1.2 \pm 0.075$ & $-0.80 \pm 0.061$ & $-0.40 \pm 0.068$ \\
\hline
\end{tabular}

\subsection{CONCLuSion}

The experimental results of measurements of Soret coefficients of both the binary and ternary systems were presented in this chapter. To validate the experimental setup and the newly designed cell, the binary mixture of THN- $\mathrm{C}_{12}$ with a mass fraction of 50/50 was used. The Soret coefficient of this mixture is available in the Fontainebleau benchmark. The experimental results showed a good agreement. 
The effect of the inclination of the cell on the separation and the concentration field were studied. The concentration fields in different inclinations were measured. The concentration profile along the vertical line at different inclinations were plotted from interferometric data. The nonlinearity of the concentration profile showed that it is necessary to have precise alignment of the cell for the measurement of the Soret coefficient.

The ternary mixtures of THN-IBB-C ${ }_{12}$ with a mass fractions of: $80 / 10 / 10,70 / 10 / 20$, $73.4 / 10.3 / 16.3,71.8 / 20.2 / 8,81.9 / 10 / 8.1$ at the mean temperature of $25{ }^{\circ} \mathrm{C}$ were studied to obtain the Soret coefficients. The results for $80 / 10 / 10$ were in good agreement with the results of the other research laboratories. The Soret coefficients of the other four compositions were obtained and proposed for the first time. 


\section{CONCLUSIONS AND FUTURE RECOMMENDATIONS}

\subsection{Conclusions}

The importance of the Soret effect or thermal diffusion in the oil industry has led to several different investigations to find the concentration field affected by the Soret effect in hydrocarbon mixtures. Finding the Soret coefficient is one of the goals of such studies. Soret coefficient is defined as the ratio of thermal diffusion coefficient to the ratio of diffusion coefficient. Thermal diffusion experiments are categorized into two main groups: convective coupling and convectionless methods. In convective coupling methods separation of the components is induced by natural convection and thermal diffusion while in the convectionless techniques, natural convection is an undesirable effect and prevents the segregation. A series of experiments were performed in space by researches in order to avoid the convective motions. Hydrocarbon mixtures were cases of study. The tested mixture was placed in a cubic cavity named Soret cell and heated from above. Interferometry setup was used as the measurement tool. Although the obtained information were reliable and accurate, but the experiments in space are too expensive.

In the present study, the convectionless ground based experiments were performed to measure thermal diffusion in hydrocarbon mixtures. However, the Soret cell used in space was not optimized for ground based experiments and it suffered from free convections in the mixture. The goal of this study was to redesign a Soret cell and overcome this problem. A set of numerical studies were done to optimize the thermal design of the cell. The numerical results showed that the temperature profile in the mixture was more linear in the new design compared to that in the previous design. More linearity of the temperature profile leads to weaker free convections.

After numerical analyses, the cell was manufactured and in the next step, experimental investigations were performed on both cells. The optical interferometry technique with MachZehnder arrangement was used to measure concentration and temperature fields. This technique is non-intrusive, which is highly beneficial to the convectionless Soret tests. Furthermore, the concentration and temperature fields can be obtained in all points of the test section at every moment of the experiment. This technique is based on the optical path difference between two 
coherent laser beams. These two beams are split from one single beam by a plano-convex lens. The objective beam traverses the test section and the reference beam passes the free environment. The variation of the index of refraction due to the concentration and temperature variations cause the optical path difference. The beams are recombined again at a second plano-convex lens, and the recombined beam is imaged as the fringe patterns that are recorded by a CCD camera. Thus, each recorded fringe pattern has information about the temperature and concentration field at the time that the image is recorded. Through image processing, the information can be extracted.

The fast Fourier transform method (FFT) was used to extract the information. The phase band obtained from FFT was unwrapped, and the variation of the index of refraction was found. The hydrocarbon mixture composed from THN and $\mathrm{C}_{12}$ was used as the binary mixture. This mixture was previously studied by other research groups and the Soret coefficients are available in the literature in a benchmark. The measured results using the new design cell are in a good agreement with the benchmark values within the experimental errors while using the space cell in ground conditions did not reveal correct results. In result, the cell was optimized successfully and made ready for further ground-based experiments.

The next goal of this study was to investigate the effect of the inclination of the cell on the separation of the components. This effect had been ignored in other studies and, based on the achieved results, it is strongly believed that deleterious motions are formed due to the misalignment of the cell. The Soret cell was installed on the optical bench at different angles to study the effect of the inclination. The numerical results showed that by inclining the cell with only half a degree of inclination, convective motions grew significantly. The experimental results showed that the separation occurred linearly when the cell is leveled while the concentration fields suggested that there were strong convections in the inclined cell. Thus, it was proved that how essential it is to align the cell properly with zero degree inclination in order to perform the experiments properly.

Finally, ternary hydrocarbon mixture composed of THN-IBB-C 12 with five different mass fractions of: $80 / 10 / 10,70 / 10 / 20,73.4 / 10.3 / 16.3,71.8 / 20.2 / 8,81.9 / 10 / 8.1$ were studied at the mean temperature of $25^{\circ} \mathrm{C}$. The new optimized cell was used to place the mixtures inside. The MachZehnder arrangement of interferometry with two laser sources with different wavelengths was used to conduct the experiments. The measured Soret coefficients of 80/10/10 were in a good agreement with the other benchmark values. It was the first time that the Soret coefficient of the other four 
mixtures were measured and the new values were proposed as a contribution to the benchmark values of Soret coefficient for hydrocarbon mixtures.

\subsection{CONTRIBUTIONS AND FUTURE WORK}

The distribution of hydrocarbon mixtures is crude oil is affected by Soret effect and it is very important to measure thermal diffusion in such mixtures. The non-intrusive-nature of the interferometry technique has made it one of the most accurate measurement techniques in convectionless studies of thermal diffusion.

In this dissertation the thermal diffusion in binary and ternary hydrocarbon mixtures was measured experimentally by using interferometry setup with Mach-Zehnder arrangement. The major contributions are:

- A new Soret cell was designed which was a re-design of a cell used in space experiments. With a more linear temperature and concentration profiles and weaker velocities compared to the space cell, the new cell is highly efficient for ground-based experiment.

- The effect of the location of the filling channel and height of the cell was studied numerically and experimentally. By moving the filling channels to the corners of the cavity, a convection free zone was established in centre of the cell. By decreasing the height of the cell, the measurement time reduced significantly.

- The effect of the inclination of the cell on the thermal diffusion was studied for the first time. This effect was not taken into account in other studies. It was shown that by inclining the cell the deleterious convective motions grew significantly.

- The optical interferometry setup with Mach-Zehnder arrangement by using two lasers with different wavelengths was established for ground-based thermal diffusion experiments on transparent ternary mixtures.

- The concentration and temperature fields for ternary hydrocarbon mixtures composed of THN, IBB, and $\mathrm{C}_{12}$ were measured experimentally for five different mass fractions.

- The Soret coefficients for ternary mixtures with four new mass fractions were measured and proposed. The positive Soret coefficient for THN indicates that this component was concentrated near the cold side while the other two components with negative Soret coefficients migrated toward the hot side. 
The following research areas are suggested for future works:

I. Establishing the ternary mixture-optical setup for ground-based experiment for the first time, a field for ternary mixtures has been opened.

II. There are more mass fraction of ternary mixture composed of THN, IBB, and $\mathrm{C}_{12}$ which has not studied yet. The study of concentration and temperature profile of this mass fraction in a Soret experiment is recommended.

III. Based on our literature survey, the temperature and concentration derivatives of refractive index (contrast factors) are not available for some mass fractions of the hydrocarbon ternary mixtures. This values are required to be able to measure concentration and temperature fields by using interferometry technique. Thus, it is necessary to measure these values in the future works.

IV. By having the contrast factors, the Soret coefficient for rest of the mass fractions of ternary hydrocarbon mixtures can be measured and a full benchmark of Soret coefficient can be provided. 


\section{REFERENCES}

[1] C. Ludwig, Diffusion zwischen ungleich erwärmten Orten gleich zusammengesetzter Lösungen, Akademie der Wissenschaften in Wien. Sitzungsberichte. Mathematischnaturwissenschaftliche Klasse., 20 (1856).

[2] J.K. Platten, P. Costesèque, Charles Soret. A short biography, Eur. Phys. J. E, 15(3) (2004) 235239.

[3] A. Firoozabadi, Thermodynamics of Hydrocarbon Reservoirs, McGraw-Hill, New York City, 1991.

[4] F. Montel, Phase equilibria needs for petroleum exploration and production industry, Fluid Phase Equilib., 84(0) (1993) 343-367.

[5] G. S., M. P., Non-equilibrium Thermodynamics, Dover Publication Inc., 1953.

[6] J.F. Torres, A. Komiya, D. Henry, S. Maruyama, Measurement of Soret and Fickian diffusion coefficients by orthogonal phase-shifting interferometry and its application to protein aqueous solutions, J. Chem. Phys., 139(7) (2013) 074203.

[7] Soret, Sur l'état d'équilibre que prend au point de vue de sa concentration une dissolution saline primitivement homogène dont deux parties sont portées à des températures différentes., Arch Sci Phys Nat, 2 (1879) 46-61.

[8] E.A. Spiegel, Convection in Stars II. Special Effects, Annual Review of Astronomy and Astrophysics, 10(1) (1972) 261-304.

[9] C. Angeli, E. Leonardi, The effect of thermodiffusion on the stability of a salinity gradient solar pond, Int. J. Heat Mass Transfer, 48(21-22) (2005) 4633-4639.

[10] R.W. Schmitt, Double Diffusion in Oceanography, Annual Review of Fluid Mechanics, 26 (1994) 255-285. 
[11] M.E. Schimpf, J.C. Giddings, Characterization of thermal diffusion in polymer solutions by thermal field-flow fractionation: effects of molecular weight and branching, Macromolecules, 20(7) (1987) 1561-1563.

[12] W. Köhler, C. Rosenauer, P. Rossmanith, Holographic grating study of mass and thermal diffusion of polystyrene/toluene solutions, Int. J. Thermophys., 16(1) (1995) 11-21.

[13] R. Kita, S. Wiegand, J. Luettmer-Strathmann, Sign change of the Soret coefficient of poly(ethylene oxide) in water/ethanol mixtures observed by thermal diffusion forced Rayleigh scattering, J. Chem. Phys., 121(8) (2004) 3874-3885.

[14] J. Janča, Micro-Thermal Field-Flow Fractionation in the Analysis of Polymers and Particles: A Review, Int. J. Polym. Anal. Charact., 11(1) (2006) 57-70.

[15] G.H. Thompson, M.N. Myers, J.C. Giddings, An Observation of a Field-Flow Fractionation Effect with Polystyrene Samples, Separation Science, 2(6) (1967) 797-800.

[16] M. Martin, M. Byoung-Ryul, M. Myeong Hee, Interpretation of thermal field-flow fractionation experiments in a tilted channel, J. Chromatogr. A, 788(1-2) (1997) 121-130.

[17] H. Cölfen, M. Antonietti, Field-Flow Fractionation Techniques for Polymer and Colloid Analysis, in: M. Schmidt (Ed.) New Developments in Polymer Analytics I, Springer Berlin Heidelberg, 2000, pp. 67-187.

[18] M.E. Schimpf, K. Caldwell, J.C. Giddings, Field-Flow Fractionation Handbook, Wiley, 2000.

[19] M. Martin, C. Van Batten, M. Hoyos, Determination of Thermodiffusion Parameters from Thermal Field-Flow Fractionation Retention Data, in: W. Köhler, S. Wiegand (Eds.) Thermal Nonequilibrium Phenomena in Fluid Mixtures, Springer Berlin Heidelberg, 2002, pp. 250-284.

[20] F.A. Messaud, R.D. Sanderson, J.R. Runyon, T. Otte, H. Pasch, S.K.R. Williams, An overview on field-flow fractionation techniques and their applications in the separation and characterization of polymers, Prog. Polym. Sci., 34(4) (2009) 351-368. 
[21] J. Janca, V. Kasparkova, V. Halabalova, L. Simek, J. Ruzicka, E. Barosova, Micro-thermal field-flow fractionation of bacteria, J Chromatogr B Analyt Technol Biomed Life Sci, 852(1-2) (2007) 512-518.

[22] V. Kašpárková, V. Halabalová, L. Šimek, J. Růžička, J. Janča, Separation of bacteria in temperature gradient: Micro-Thermal Focusing Field-Flow Fractionation, J. Biochem. Bioph. Methods, 70(4) (2007) 685-687.

[23] C. Van Batten, M. Hoyos, M. Martin, Thermal field-flow fractionation of colloidal materials: Methylmethacrylate-styrene linear di-block copolymers, Chromatographia, 45(1) (1997) 121-126.

[24] J. Janca, Micro - channel Thermal Field - Flow Fractionation: High - Speed Analysis of Colloidal Particles, Journal of Liquid Chromatography \& Related Technologies, 26(6) (2003) 849869.

[25] L. Pasti, S. Agnolet, F. Dondi, Thermal Field-Flow Fractionation of Charged Submicrometer Particles in Aqueous Media, Anal. Chem., 79(14) (2007) 5284-5296.

[26] Q.T. Pham, Modelling heat and mass transfer in frozen foods: a review, International Journal of Refrigeration, 29(6) (2006) 876-888.

[27] S.f.L.O. Bonner Fj, Thermal diffusion as a mechanism for biological transport, Zeitschrift für Naturforschung. Section C: Biosciences, 39(6) (1984) 656-661.

[28] P.W.C.J.R.P.D.S.B.D. Baaske, Optical Thermophoresis for Quantifying the Buffer Dependence of Aptamer Binding, ANIE Angewandte Chemie International Edition, 49(12) (2010) 2238-2241.

[29] L.A. Braun D, Trapping of DNA by thermophoretic depletion and convection, Phys. Rev. Lett., 89(18) (2002).

[30] A. Firoozabadi, Thermodynamics of hydrocarbon reservoirs, McGraw-Hill, 1999. 
[31] P. Costesèque, D. Fargue, P. Jamet, Thermodiffusion in Porous Media and Its Consequences, in: W. Köhler, S. Wiegand (Eds.) Thermal Nonequilibrium Phenomena in Fluid Mixtures, Springer Berlin Heidelberg, 2002, pp. 389-427.

[32] P. Costeseque, M.E. Maataoui, J. Garrigues, Analysis try of relationship between thermodiffusional mobility and molecular intrinsic properties of hydrocarbon species, by artificial neuronal networks, in the case of compound mixtures (crude, Entropie, 32(198) (1996) 15-24.

[33] P. Costeseque, M.E. Maataoui, E. Riviere, Selective enrichments induced by thermogravitational diffusion in porous medium on hydrocarbon species of crude oil the specific case of paraffin isomers, Entropie, 30(184) (1994) 94-100.

[34] P. Costeseque, M.E. Maataoui, On differentiation of hydrocarbons in oil-fiels fluids submitted to thethermogravitational effect in two-phase oil-water packed colums, Entropie, 30(184) (1994) 101-107.

[35] J.K. Platten, The Soret Effect: A Review of Recent Experimental Results, Journal of Applied Mechanics, 73(1) (2005) 5-15.

[36] K. Clusius, G. Dickel, Neues Verfahren zur Gasentmischung und Isotopentrennung, Naturwissenschaften, 26(33) (1938) 546-546.

[37] A. Mialdun, V. Yasnou, V. Shevtsova, A. Königer, W. Köhler, D. Alonso de Mezquia, M.M. Bou-Ali, A comprehensive study of diffusion, thermodiffusion, and Soret coefficients of waterisopropanol mixtures, The Journal of Chemical Physics, 136(24) (2012) 244512.

[38] F.H. Horne, R.J. Pearman, Thermogravitational thermal diffusion in liquids. II. Experimental thermal diffusion factors for carbon tetrachloride-cyclohexane at 25 C, J. Chem. Phys., 37(12) (1962) 2857-2872.

[39] D.J. Trevoy, H.G. Drickamer, Thermal diffusion in binary liquid hydrocarbon mixtures, J. Chem. Phys., 17 (1949) 1120-1124.

[40] C.J. Danby, J.D. Lambert, C.M. Mitchell, Separation of hydrocarbon isomers by thermal diffusion, Nature, 177 (1956) 1225-1226. 
[41] J.F. Dutrieux, J.K. Platten, G. Chavepeyer, M.M. Bou-Ali, On the measurement of positive Soret coefficients, J. Phys. Chem. B, 106(23) (2002) 6104-6114.

[42] P. Blanco, M.M. Bou-Ali, J.K. Platten, J.A. Madariaga, P. Urteaga, C. Santamaria, Thermodiffusion coefficient for binary liquid hydrocarbon mixtures, J. Non-Equilib. Thermodyn., 32(3) (2007) 309-317.

[43] P. Costeseque, J.C. Loubet, Measuring the Soret coefficient of binary hydrocarbon mixtures in packed thermogravitational columns, Philos. Mag., 83(17-18) (2003) 2017-2022.

[44] J.K. Platten, M.M. Bou-Ali, J.F. Dutrieux, Enhanced molecular separation in inclined thermogravitational columns, J. Phys. Chem. B, 107(42) (2003) 11763-11767.

[45] R.L. Saxton, E.L. Dougherty, H.G. Drickamer, Thermal diffusion in binary liquid mixtures of molecules of simple symmetry, J. Chem. Phys., 22 (1954) 1166-1168.

[46] J.C.R. Turner, Thermal diffusion with laminar flow in a duct: A theoretical solution and practical results, Chem. Eng. Sci., 17(2) (1962) 95-103.

[47] B.D. Butler, J.C.R. Turner, Flow-cell studies of thermal diffusion in liquids. II. Phenomenological equations and their solution, Trans. Faraday Society, 62(11) (1966) 3121-3130.

[48] J.C.R. Turner, B.D. Butler, M.J. Story, Flow-cell studies of thermal diffusion in liquids. III. The CC14+cyclohexane system, Trans. Faraday Society, 63(8) (1967) 1906-1912.

[49] M.J. Story, J.C.R. Turner, Flow-cell studies of thermal diffusion in liquids. V. Binary mixtures of $\mathrm{CH} 3 \mathrm{OH}$ with $\mathrm{CCl}$, benzene and cyclohexane at 25C, Trans. Faraday Society, 65(6) (1969) 1523-1529.

[50] V.S. Van, J.C. Legros, Soret coefficients of organic solutions measured in the microgravity SCM experiment and by the flow and Benard cells, J. Phys. Chem. B, 102(22) (1998) 4426-4431.

[51] S. Chandrasekhar, Hydrodynamic and Hydromagnetic Stability, Oxford University Press, 1961. 
[52] M. Giglio, A. Vendramini, Thermal lens effect in a binary liquid mixture: A new effect, Appl. Phys. Lett., 25(10) (1974) 555-557.

[53] V. Degiorgio, M. Giglio, A. Vendramini, Transient and steady-state behavior of the Soretdriven instability. Analogy with the laser instability, in: Statistical Physics-Statphys 13, 24-30 Aug. 1977, Adam Hilger Ltd., Bristol, UK, 1978, pp. 752-755.

[54] M. Giglio, A. Vendramini, Thermal-diffusion measurements near a consolute critical point, Phys. Rev. Lett., 34(10) (1975) 561-564.

[55] P. Kolodner, H. Williams, C. Moe, Optical measurement of the Soret coefficient of ethanol/water solutions, J. Chem. Phys., 88(10) (1988) 6512-6524.

[56] K.J. Zhang, M.E. Briggs, R.W. Gammon, J.V. Sengers, Optical measurement of the Soret coefficient and the diffusion coefficient of liquid mixtures, J. Chem. Phys., 104(17) (1996) 68816892.

[57] S. Wiegand, W. Köhler, Measurement of Transport Coefficients by an Optical Grating Technique, in: W. Köhler, S. Wiegand (Eds.) Thermal Nonequilibrium Phenomena in Fluid Mixtures, Springer Berlin Heidelberg, 2002, pp. 189-210.

[58] A. Mialdun, V.M. Shevtsova, Development of optical digital interferometry technique for measurement of thermodiffusion coefficients, Int. J. Heat Mass Transfer, 51(11-12) (2008) 31643178 .

[59] A. Mialdun, V. Shevtsova, Open Questions on Reliable Measurements of Soret Coefficients, Microgravity Science and Technology, 21(1-2) (2009) 31-36.

[60] J.K. Platten, M.M. Bou-Ali, P. Costesèque, J.F. Dutrieux, W. Köhler, C. Leppla, S. Wiegand, G. Wittko, Benchmark values for the Soret, thermal diffusion and diffusion coefficients of three binary organic liquid mixtures, Philos. Mag., 83(17-18) (2003) 1965-1971.

[61] J.K. Platten, M.M. Bou-Ali, J.F. Dutrieux, Precise determination of the Soret, thermodiffusion and isothermal diffusion coefficients of binary mixtures of dodecane, isobutylbenzene and 1,2,3,4- 
tetrahydronaphthalene (contribution of the University of Mons to the benchmark test), Philos. Mag., 83(17-18) (2003) 2001-2010.

[62] G. Wittko, W. Köhler, Precise determination of the Soret, thermal diffusion and mass diffusion coefficients of binary mixtures of dodecane, isobutylbenzene and 1,2,3,4-tetrahydronaphthalene by a holographic grating technique, Philos. Mag., 83(17-18) (2003) 1973-1987.

[63] C. Leppla, S. Wiegand, Investigation of the Soret effect in binary liquid mixtures by thermaldiffusion-forced Rayleigh scattering (contribution to the benchmark test), Philos. Mag., 83(17-18) (2003) 1989-1999.

[64] P. Costesèque, J.-C. Loubet, Measuring the Soret coefficient of binary hydrocarbon mixtures in packed thermogravitational columns (contribution of Toulouse University to the benchmark test), Philos. Mag., 83(17-18) (2003) 2017-2022.

[65] A. Mialdun, V. Shevtsova, Measurement of the Soret and diffusion coefficients for benchmark binary mixtures by means of digital interferometry, J. Chem. Phys., 134(4) (2011) 044524.

[66] A. Königer, B. Meier, W. Köhler, Measurement of the Soret, diffusion, and thermal diffusion coefficients of three binary organic benchmark mixtures and of ethanol-water mixtures using a beam deflection technique, Philos. Mag., 89(10) (2009) 907-923.

[67] A. Leahy-Dios, M.M. Bou-Ali, J.K. Platten, A. Firoozabadi, Measurements of molecular and thermal diffusion coefficients in ternary mixtures, J. Chem. Phys., 122(23) (2005).

[68] J.P. Larre, J.K. Platten, G. Chavepeyer, Soret effects in ternary systems heated from below, Int. J. Heat Mass Transfer, 40(3) (1997) 545-555.

[69] S. Vanvaerenbergh, S. Srinivasan, M.Z. Saghir, Thermodiffusion in multicomponent hydrocarbon mixtures: Experimental investigations and computational analysis, J. Chem. Phys., 131(11) (2009).

[70] K.B. Haugen, A. Firoozabadi, On measurement of molecular and thermal diffusion coefficients in multicomponent mixtures, J. Phys. Chem. B, 110(35) (2006) 17678-17682. 
[71] A. Koniger, H. Wunderlich, W. Kohler, Measurement of diffusion and thermal diffusion in ternary fluid mixtures using a two-color optical beam deflection technique, J. Chem. Phys., 132(17) (2010) 174506 (174508 pp.).

[72] A. Ahadi, S. Van Varenbergh, M.Z. Saghir, Measurement of the Soret coefficients for a ternary hydrocarbon mixture in low gravity environment, J. Chem. Phys., 138(20) (2013) 204201 (204217 pp.).

[73] A. Mialdun, V. Shevtsova, Communication: New approach for analysis of thermodiffusion coefficients in ternary mixtures, J. Chem. Phys., 138(16) (2013).

[74] P. Blanco, M.M. Bou-Ali, J.K. Platten, D.A. de Mezquia, J.A. Madariaga, C. Santamaria, Thermodiffusion coefficients of binary and ternary hydrocarbon mixtures, J. Chem. Phys., 132(11) (2010) 114506 (114506 pp.).

[75] M.M. Bou-Ali, A. Ahadi, D. Alonso de Mezquia, Q. Galand, M. Gebhardt, O. Khlybov, W. Köhler, M. Larrañaga, J.C. Legros, T. Lyubimova, A. Mialdun, I. Ryzhkov, M.Z. Saghir, V. Shevtsova, S. Van Varenbergh, Benchmark DCMIX1: Soret, thermodiffusion and molecular diffusion coefficients of the ternary mixture THN-IBB-nC12, European Physical Journal E, (In press).

[76] E.R.G. Eckert, R.J. Goldstein, Measurement techniques in heat transfer, Technivision Services, England, 1970.

[77] V. Shevtsova, V. Sechenyh, A. Nepomnyashchy, J.C. Legros, Analysis of the application of optical two-wavelength techniques to measurement of the Soret coefficients in ternary mixtures, Philos. Mag., 91(26) (2011) 3498-3518.

[78] M.A. Rahman, M.Z. Saghir, Ground based measurement and theoretical calculation of Soret coefficient of binary hydrocarbon mixtures, Exp. Therm Fluid Sci., 49 (2013) 31-39.

[79] T. Kreis, Digital holographic interference-phase measurement using the Fourier-transform method, Journal of the Optical Society of America A (Optics and Image Science), 3(6) (1986) 847855. 
[80] W.W. Macy, Jr., Two-dimensional fringe-pattern analysis, Appl. Opt., 22(23) (1983) 38983901.

[81] C. Mattisson, D. Karlsson, S.G. Pettersson, G. Zacchi, A. Axelsson, Light deflection and convection in diffusion experiments using holographic interferometry, Journal of Physics D (Applied Physics), 34(20) (2001) 3088-3096.

[82] A. Mialdun, D. Melnikov, V. Shevtsova, Observation of diffusion phenomena in ground experiments: Problems and solutions, in: AIAA 57th International Astronautical Congress, IAC 2006, October 2, 2006 - October 6, 2006, American Institute of Aeronautics and Astronautics Inc., Valencia, Spain, 2006, pp. 729-740.

[83] T.L. Bergman, F.P. Incropera, D.P. DeWitt, A.S. Lavine, Fundamentals of Heat and Mass Transfer, Wiley, 2011.

[84] V.V. Sechenyh, J.-C. Legros, V. Shevtsova, Optical properties of binary and ternary liquid mixtures containing tetralin, isobutylbenzene and dodecane, J. Chem. Thermodyn., 62 (2013) 6468.

[85] M. Yahya, M.Z. Saghir, Prediction and Experimental Measurement of Refractive Index in Ternary Hydrocarbon Mixtures, Journal of Chemical \& Engineering Data, 60(8) (2015) 23292342.

[86] D. Alonso de Mezquia, M. Larrañaga, M.M. Bou-Ali, J.A. Madariaga, C. Santamaría, J.K. Platten, Contribution to thermodiffusion coefficient measurements in DCMIX project, International Journal of Thermal Sciences, 92 (2015) 14-16.

[87] Q. Galand, S. Van Vaerenbergh, Contribution to the benchmark for ternary mixtures: Measurement of diffusion and Soret coefficients of ternary system tetrahydronaphtaleneisobutylbenzene-n-dodecane with mass fractions 80-10-10 at 25 C, European Physical Journal E, 38(4) (2015).

[88] M. Gebhardt, W. Kohler, Contribution to the benchmark for ternary mixtures: Measurement

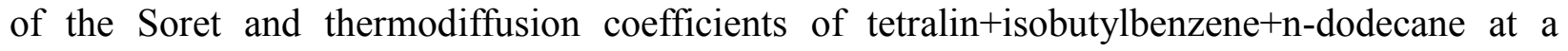


composition of $(0.8 / 0.1 / 0.1)$ mass fractions by two-color optical beam deflection, European Physical Journal E, 38(4) (2015).

[89] O. Khlybov, I. Ryzhkov, T. Lyubimova, Contribution to the benchmark for ternary mixtures: Measurement of diffusion and Soret coefficients in 1,2,3,4-tetrahydronaphthalene, isobutylbenzene, and dodecane onboard the ISS, Eur. Phys. J. E, 38(4) (2015) 1-16.

[90] M. Larrañaga, M.M. Bou-Ali, D. de Mezquia, D.A. Rees, J. Madariaga, C. Santamaría, J. Platten, Contribution to the benchmark for ternary mixtures: Determination of Soret coefficients by the thermogravitational and the sliding symmetric tubes techniques, Eur. Phys. J. E, 38(4) (2015) 1-7.

[91] A. Mialdun, J.C. Legros, V. Yasnou, V. Sechenyh, V. Shevtsova, Contribution to the benchmark for ternary mixtures: Measurement of the Soret, diffusion and thermodiffusion coefficients in the ternary mixture THN/IBB/nCinf12/inf with 0.8/0.1/0.1 mass fractions in ground and orbital laboratories, European Physical Journal E, 38(4) (2015). 